\title{
A COUPLED MIXED FINITE ELEMENT METHOD FOR THE INTERACTION PROBLEM BETWEEN AN ELECTROMAGNETIC FIELD AND AN ELASTIC BODY*
}

\author{
GABRIEL N. GATICA ${ }^{\dagger}$, GEORGE C. HSIAO , AND SALIM MEDDAHI $^{\S}$
}

\begin{abstract}
This paper deals with the coupled problem arising from the interaction of a timeharmonic electromagnetic field with a three-dimensional elastic body. More precisely, we consider a suitable transmission problem holding between the solid and a sufficiently large annular region surrounding it, and aim to compute both the magnetic component of the scattered wave and the stresses that take place in the obstacle. To this end, we assume Voigt's model, which allows interaction only through the boundary of the body, and employ a dual-mixed variational formulation in the solid medium. As a consequence, one of the two transmission conditions becomes essential, whence it is enforced weakly through the introduction of a Lagrange multiplier. An abstract framework developed recently, which is based on regular decompositions of the spaces involved, is applied next to show that our coupled variational formulation is well-posed. In addition, we define the corresponding Galerkin scheme by using PEERS in the solid and using the edge finite elements of Nédélec in the electromagnetic region. Then, we prove that the resulting coupled mixed finite element scheme is uniquely solvable and convergent. Moreover, optimal a priori error estimates are derived in the usual way. Finally, some numerical results illustrating the analysis and the good performance of the method are also reported.
\end{abstract}

Key words. Maxwell equations, edge finite elements, elastodynamics equations, PEERS

AMS subject classifications. 65N30, 65N12, 65N15, 74F10, 74B05, 35J05

DOI. $10.1137 / 090754212$

1. Introduction. A successful strategy has been developed in [4] to analyze, at the continuous and discrete levels, a class of variational formulations defined by noncoercive bilinear (or sesquilinear) forms. More precisely, though the analysis in [4] was originally motivated by the study of Maxwell equations, the author succeeded in setting up the corresponding technique in a quite general framework. In fact, the key issue is the utilization of a Helmholtz-type decomposition of the main unknown, which allows us to reveal hidden compactness properties of the formulation, and hence the classical results connecting Fredholm alternative and projection methods (see, e.g., [18], [21]) can be applied straightforwardly.

The method from [4] was applied recently in [12] and [14] to deal with a timeharmonic fluid-solid interaction problem posed in the plane. The model consists of an elastic body occupying a region $\Omega_{s}$, which is subject to a given incident acoustic wave that travels in the fluid surrounding it. In [12] the fluid is supposed to occupy an annular region $\Omega_{f}$, and a Robin boundary condition imitating the behavior of the

* Received by the editors March 30, 2009; accepted for publication (in revised form) June 1, 2010; published electronically August 24, 2010. This research was partially supported by FONDAP and BASAL projects CMM, Universidad de Chile, by Centro de Investigación en Ingeniería Matemática $\left(\mathrm{CI}^{2} \mathrm{MA}\right)$, Universidad de Concepción, and by the Ministry of Education of Spain through the project MTM2007-65088.

http://www.siam.org/journals/sinum/48-4/75421.html

${ }^{\dagger} \mathrm{CI}^{2} \mathrm{MA}$ and Departamento de Ingeniería Matemática, Universidad de Concepción, Casilla 160-C, Concepción, Chile (ggatica@ing-mat.udec.cl).

${ }^{\ddagger}$ Department of Mathematical Sciences, University of Delaware, Newark, DE 19716 (hsiao@math. udel.edu).

$\S$ Departamento de Matemáticas, Universidad de Oviedo, Calvo Sotelo s/n, Oviedo, Spain (salim@ uniovi.es). 
scattered field at infinity is imposed on its exterior boundary $\Gamma$. On the other hand, instead of using an approximate boundary condition on $\Gamma$, the approach in [14] considers a nonlocal absorbing boundary condition based on integral equations defined on $\Gamma$. In this way, a combined double and single layer potential representation of the scattered wave allows one to incorporate the far field effects into the continuous and Galerkin formulations. In any case, the usual primal formulation in the fluid region $\Omega_{f}$ and a dual-mixed variational formulation for plane elasticity in the obstacle $\Omega_{s}$ are employed in both works. Actually, in contrast to the usual dual-mixed approach, the elastodynamic equation is used here to eliminate the displacement, which yields the stress tensor as the main unknown in the solid $\Omega_{s}$. As a consequence, the noncompactness of the imbedding $\mathbf{H}\left(\operatorname{div} ; \Omega_{s}\right) \hookrightarrow\left[L^{2}\left(\Omega_{s}\right)\right]^{2}$ motivates, following the original idea from [4], the introduction of a suitable decomposition of $\mathbf{H}\left(\mathbf{d i v} ; \Omega_{s}\right)$, whereas the compactness of the imbedding $H^{1}\left(\Omega_{f}\right) \hookrightarrow L^{2}\left(\Omega_{f}\right)$ simplifies the analysis of the terms defined on $\bar{\Omega}_{f}$ (since the Fredholm alternative arises naturally there), and then no further decomposition is needed. The corresponding discrete schemes are defined with PEERS elements in $\Omega_{s}$ and the traditional Lagrange finite elements in $\bar{\Omega}_{f}$. The stability and convergence of these Galerkin methods also rely on a stable decomposition of the finite element subspace used to approximate the stress unknown.

The purpose of the present work is to further apply the approach from [4] and [12] to the transmission problem arising from the interaction of a three-dimensional (3D) elastic body with a time-harmonic electromagnetic field. As we will see below, the corresponding analysis will have to deal with several additional technical difficulties arising from the 3D setting and the incorporation of the Maxwell equations instead of the Helmholtz equation. As in [12], we assume here that the electromagnetic field occupies an annular region $\Omega_{m}$ on whose exterior boundary $\Gamma$ a condition compatible with the behavior of the scattered field at infinity is imposed. In addition, according to Voigt's model (see, e.g., [10] for details), we discard the eventual penetration of the electromagnetic field inside the body and assume that the interaction between both media is governed only by the equilibrium of tangential forces along the interface $\partial \Omega_{s}$. Hence, our aim is to provide and analyze a corresponding coupled mixed finite element method that permits us to compute the scattered electromagnetic wave and the stresses of the solid. However, we will observe that the noncompactness of the imbeddings $\mathbf{H}\left(\operatorname{div} ; \Omega_{s}\right) \hookrightarrow\left[L^{2}\left(\Omega_{s}\right)\right]^{3}$ and $\mathbf{H}\left(\mathbf{c u r l} ; \Omega_{m}\right) \hookrightarrow\left[L^{2}\left(\Omega_{m}\right)\right]^{3}$ stops us from employing a Fredholm alternative for the original form of the resulting variational formulation. In order to overcome this difficulty, we follow again the technique developed in [4] and introduce now suitable decompositions of both $\mathbf{H}\left(\mathbf{d i v} ; \Omega_{s}\right)$ and $\mathbf{H}\left(\operatorname{curl} ; \Omega_{m}\right)$. This differs from the analysis in [12], where only the decomposition of the first space was needed. The corresponding Galerkin scheme is defined with PEERS in the solid $\Omega_{s}$ and with the edge finite elements of Nédélec in the electromagnetic region $\Omega_{m}$, and hence stable decompositions of these finite element subspaces allow us to prove the associated stability and convergence of the discrete method.

The rest of the paper is organized as follows. In section 2 we collect some known results on tangential trace operators in a generic space $\mathbf{H}(\mathbf{c u r l} ; \Omega)$. In sections 3 and 4 we describe the interaction problem and derive its coupled variational formulation. The approach from [4] is employed in section 5 to show that the continuous problem is well-posed. The corresponding Galerkin scheme is introduced and analyzed in sections 6 and 7. Finally, numerical results illustrating our analysis are reported in section 8 .

We end this section with some notation to be used below. Since in what follows we deal with complex valued functions, we let $\mathbb{C}$ be the set of complex numbers, use the 
symbol $\imath$ for $\sqrt{-1}$, and denote by $\bar{z}$ and $|z|$ the conjugate and modulus, respectively, of each $z \in \mathbb{C}$. In addition, given any Hilbert space $U$, we let $[U]^{3}$ and $[U]^{3 \times 3}$ denote, respectively, the space of vectors and tensors of order 3 with entries in $U$. When no confusion arises, we simply use $U^{3}$ and $U^{3 \times 3}$ instead of $[U]^{3}$ and $[U]^{3 \times 3}$, respectively. In particular, $\mathbf{I}$ is the identity matrix of $\mathbb{C}^{3 \times 3}$, and given $\boldsymbol{\tau}:=\left(\tau_{i j}\right)$ and $\zeta:=\left(\zeta_{i j}\right) \in \mathbb{C}^{3 \times 3}$, we define as usual the transpose tensor $\tau^{\mathrm{t}}:=\left(\tau_{j i}\right)$, the trace $\operatorname{tr}(\boldsymbol{\tau}):=\sum_{i=1}^{3} \tau_{i i}$, the tensor product $\boldsymbol{\tau}: \boldsymbol{\zeta}:=\sum_{i, j=1}^{3} \tau_{i j} \zeta_{i j}$, and the conjugate tensor $\bar{\tau}:=\left(\bar{\tau}_{i j}\right)$. Finally, in what follows we utilize the standard terminology for Sobolev spaces and norms, employ $\mathbf{0}$ to denote a generic null vector, and use $C$, with or without subscripts, to denote generic constants independent of the discretization parameters, which may take different values at different places.

2. Preliminaries. We denote by $\Omega \subset \mathbb{R}^{3}$ a generic bounded polyhedral domain and let $\boldsymbol{n}$ be the outward normal vector on its boundary $\Sigma$. We recall that

$$
\mathbf{H}(\operatorname{curl} ; \Omega):=\left\{\boldsymbol{W} \in\left[L^{2}(\Omega)\right]^{3}: \quad \operatorname{curl}(\boldsymbol{W}) \in\left[L^{2}(\Omega)\right]^{3}\right\}
$$

endowed with the norm $\|\boldsymbol{W}\|_{\mathbf{H}(\operatorname{curl}, \Omega)}^{2}:=\|\boldsymbol{W}\|_{\left[L^{2}(\Omega)\right]^{3}}^{2}+\|\operatorname{curl}(\boldsymbol{W})\|_{\left[L^{2}(\Omega)\right]^{3}}^{2}$ is a Hilbert space and that $\left[\mathcal{C}^{\infty}(\bar{\Omega})\right]^{3}$ is dense in $\mathbf{H}(\operatorname{curl} ; \Omega)$. As usual, $\operatorname{curl}(\boldsymbol{W})$ stands for the vector defined formally by $\nabla \times \boldsymbol{W}$. We also recall that

$$
\mathbf{H}(\operatorname{div} ; \Omega):=\left\{\boldsymbol{\tau} \in\left[L^{2}(\Omega)\right]^{3 \times 3}: \quad \operatorname{div}(\boldsymbol{\tau}) \in\left[L^{2}(\Omega)\right]^{3}\right\},
$$

endowed with the norm $\|\boldsymbol{\tau}\|_{\mathbf{H}(\mathbf{d i v} ; \Omega)}^{2}:=\|\boldsymbol{\tau}\|_{\left[L^{2}(\Omega)\right]^{3 \times 3}}^{2}+\|\operatorname{div}(\boldsymbol{\tau})\|_{\left[L^{2}(\Omega)\right]^{3}}^{2}$ is a Hilbert space and that $\left[\mathcal{C}^{\infty}(\bar{\Omega})\right]^{3 \times 3}$ is dense in $\mathbf{H}(\operatorname{div} ; \Omega)$. Here, div stands for the usual divergence operator div acting on each row of the tensor $\boldsymbol{\tau}$. It is well known that the mapping

$$
\begin{aligned}
\gamma_{\boldsymbol{n}}:\left[\mathcal{C}^{\infty}(\bar{\Omega})\right]^{3 \times 3} & \longrightarrow\left[L^{2}(\Sigma)\right]^{3}, \\
\boldsymbol{\tau} & \longrightarrow \gamma_{\boldsymbol{n}}(\boldsymbol{\tau}):=\left.\boldsymbol{\tau}\right|_{\Sigma} \boldsymbol{n}
\end{aligned}
$$

can be extended to define a normal trace operator

$$
\begin{aligned}
\gamma_{\boldsymbol{n}}: \mathbf{H}(\operatorname{div} ; \Omega) & \longrightarrow\left[H^{-1 / 2}(\Sigma)\right]^{3}, \\
\boldsymbol{\tau} & \longrightarrow \gamma_{\boldsymbol{n}}(\boldsymbol{\tau}),
\end{aligned}
$$

which is bounded, is surjective, and possesses a right inverse.

Tangential traces of functions in $\mathbf{H}(\mathbf{c u r l} ; \Omega)$ are also well understood even in the case of polyhedral domains, thanks to the recent results of [5], [6]. We give here a brief summary of these fundamental tools. To this end, we begin by defining the space

$$
\mathbf{L}_{\mathbf{t}}^{2}(\Sigma):=\left\{\boldsymbol{\mu} \in\left[L^{2}(\Sigma)\right]^{3}: \quad \boldsymbol{\mu} \cdot \boldsymbol{n}=0\right\}
$$

and the tangential trace mapping

$$
\begin{aligned}
\gamma_{\mathbf{t}}:\left[\mathcal{C}^{\infty}(\bar{\Omega})\right]^{3} & \rightarrow \mathbf{L}_{\mathbf{t}}^{2}(\Sigma), \\
\boldsymbol{v} & \mapsto \gamma_{\mathbf{t}}(\boldsymbol{v}):=\left.\boldsymbol{v}\right|_{\Sigma} \times \boldsymbol{n},
\end{aligned}
$$

together with the tangential projection operator

$$
\begin{aligned}
\boldsymbol{\pi}_{\mathbf{t}}:\left[\mathcal{C}^{\infty}(\bar{\Omega})\right]^{3} & \rightarrow \mathbf{L}_{\mathbf{t}}^{2}(\Sigma), \\
\boldsymbol{v} & \mapsto \boldsymbol{\pi}_{\mathbf{t}}(\boldsymbol{v}):=\boldsymbol{n} \times\left(\left.\boldsymbol{v}\right|_{\Sigma} \times \boldsymbol{n}\right) .
\end{aligned}
$$

Copyright (c) by SIAM. Unauthorized reproduction of this article is prohibited. 
Because of the orthogonality condition defining $\mathbf{L}_{\mathbf{t}}^{2}(\Sigma)$, this subspace of $\left[L^{2}(\Sigma)\right]^{3}$ is considered in what follows as a space of two-dimensional (2D) tangent fields.

Let us now introduce the spaces

$$
\mathbf{H}_{\perp}^{1 / 2}(\Sigma):=\gamma_{\mathbf{t}}\left(\left[H^{1}(\Omega)\right]^{3}\right) \quad \text { and } \quad \mathbf{H}_{\|}^{1 / 2}(\Sigma):=\boldsymbol{\pi}_{\mathbf{t}}\left(\left[H^{1}(\Omega)\right]^{3}\right),
$$

which are endowed with the natural Hilbert space structure that makes both $\gamma_{\mathbf{t}}$ : $\left[H^{1}(\Omega)\right]^{3} \rightarrow \mathbf{H}_{\perp}^{1 / 2}(\Sigma)$ and $\boldsymbol{\pi}_{\mathbf{t}}:\left[H^{1}(\Omega)\right]^{3} \rightarrow \mathbf{H}_{\|}^{1 / 2}(\Sigma)$ bounded and surjective. Similarly, for any $\delta \in(0,1)$, we define

$$
\mathbf{H}_{\|}^{\delta}(\Sigma):=\boldsymbol{\pi}_{\mathbf{t}}\left(\left[H^{\delta+1 / 2}(\Omega)\right]^{3}\right)
$$

and provide it with an inner product that renders $\boldsymbol{\pi}_{\mathbf{t}}:\left[H^{\delta+1 / 2}(\Omega)\right]^{3} \rightarrow \mathbf{H}_{\|}^{\delta}(\Sigma)$ continuous. We refer to [5] for an explicit definition of these spaces in the case of Lipschitz boundaries with piecewise smooth components. In the following, we will also write $\gamma_{\mathbf{t}}(\varphi)$ (or $\pi_{\mathbf{t}}(\varphi)$ ) for $\varphi \in\left[H^{1 / 2}(\Sigma)\right]^{3}$, which should be understood as $\gamma_{\mathbf{t}}\left(\gamma^{-1}(\boldsymbol{\varphi})\right)$ (or $\boldsymbol{\pi}_{\mathbf{t}}\left(\gamma^{-1} \boldsymbol{\varphi}\right)$ ), where $\gamma^{-1}:\left[H^{1 / 2}(\Sigma)\right]^{3} \rightarrow\left[H^{1}(\Omega)\right]^{3}$ is a given bounded right-inverse of the usual trace operator $\gamma:\left[H^{1}(\Omega)\right]^{3} \rightarrow\left[H^{1 / 2}(\Sigma)\right]^{3}$.

Next, we introduce the dual $\mathbf{H}_{\perp}^{-1 / 2}(\Sigma)$ of $\mathbf{H}_{\perp}^{1 / 2}(\Sigma)$ and the dual $\mathbf{H}_{\|}^{-1 / 2}(\Sigma)$ of $\mathbf{H}_{\|}^{1 / 2}(\Sigma)$ with respect to the pivot space $\mathbf{L}_{\mathbf{t}}^{2}(\Sigma)$. Then, it is easy to deduce from the Green formula

$$
\int_{\Omega}\{\boldsymbol{u} \cdot \operatorname{curl}(\boldsymbol{v})-\boldsymbol{v} \cdot \operatorname{curl}(\boldsymbol{u})\}=\int_{\Sigma} \gamma_{\mathbf{t}}(\boldsymbol{u}) \cdot \boldsymbol{\pi}_{\mathbf{t}}(\boldsymbol{v}) \quad \forall \boldsymbol{u}, \boldsymbol{v} \in\left[\mathcal{C}^{\infty}(\bar{\Omega})\right]^{3}
$$

and the fact that $\left[\mathcal{C}^{\infty}(\bar{\Omega})\right]^{3}$ is dense in $\mathbf{H}(\operatorname{curl} ; \Omega)$ that $\gamma_{\mathbf{t}}$ and $\boldsymbol{\pi}_{\mathbf{t}}$ can be extended to define bounded tangential mappings from $\mathbf{H}(\operatorname{curl} ; \Omega)$ onto $\mathbf{H}_{\|}^{-1 / 2}(\Sigma)$ and from $\mathbf{H}(\operatorname{curl} ; \Omega)$ onto $\mathbf{H}_{\perp}^{-1 / 2}(\Sigma)$, respectively. A more precise result is given by the following theorem (see [7]) (we refer to [5], [7] for the definition of the differential operators $\operatorname{div}_{\Sigma}$ and $\operatorname{curl}_{\Sigma}$ on piecewise smooth Lipschitz boundaries).

THEOREM 2.1. Let

$$
\mathbf{H}^{-1 / 2}\left(\operatorname{div}_{\Sigma} ; \Sigma\right):=\left\{\boldsymbol{\mu} \in \mathbf{H}_{\|}^{-1 / 2}(\Sigma): \quad \operatorname{div}_{\Sigma}(\boldsymbol{\mu}) \in H^{-1 / 2}(\Sigma)\right\}
$$

and

$$
\mathbf{H}^{-1 / 2}\left(\operatorname{curl}_{\Sigma} ; \Sigma\right):=\left\{\boldsymbol{\mu} \in \mathbf{H}_{\perp}^{-1 / 2}(\Sigma): \quad \operatorname{curl}_{\Sigma}(\boldsymbol{\mu}) \in H^{-1 / 2}(\Sigma)\right\}
$$

Then

$$
\gamma_{\mathbf{t}}: \mathbf{H}(\mathbf{c u r l} ; \Omega) \rightarrow \mathbf{H}^{-1 / 2}\left(\operatorname{div}_{\Sigma} ; \Sigma\right) \quad \text { and } \quad \boldsymbol{\pi}_{\mathbf{t}}: \mathbf{H}(\operatorname{curl} ; \Omega) \rightarrow \mathbf{H}^{-1 / 2}\left(\operatorname{curl}_{\Sigma} ; \Sigma\right)
$$

are bounded, are surjective, and possess continuous right-inverses. Moreover, the $\left[L^{2}(\Sigma)\right]^{3}$-inner product can be extended to define a duality product $\langle\cdot, \cdot\rangle_{\mathbf{t}, \Sigma}$ between the spaces $\mathbf{H}^{-1 / 2}\left(\operatorname{div}_{\Sigma} ; \Sigma\right)$ and $\mathbf{H}^{-1 / 2}\left(\operatorname{curl}_{\Sigma} ; \Sigma\right)$.

As a consequence of this theorem, Green's formula (2.2) can be extended to functions $\boldsymbol{u}, \boldsymbol{v}$ in $\mathbf{H}(\mathbf{c u r l} ; \Omega)$ if the boundary integral of the right-hand side is interpreted as $\left\langle\gamma_{\mathbf{t}}(\boldsymbol{u}), \boldsymbol{\pi}_{\mathbf{t}}(\boldsymbol{v})\right\rangle_{\mathbf{t}, \Sigma}$, that is,

$$
\int_{\Omega}\{\boldsymbol{u} \cdot \operatorname{curl}(\boldsymbol{v})-\boldsymbol{v} \cdot \operatorname{curl}(\boldsymbol{u})\}=\left\langle\gamma_{\mathbf{t}}(\boldsymbol{u}), \boldsymbol{\pi}_{\mathbf{t}}(\boldsymbol{v})\right\rangle_{\mathbf{t}, \Sigma} \quad \forall \boldsymbol{u}, \boldsymbol{v} \in \mathbf{H}(\operatorname{curl} ; \Omega) .
$$

In addition, exchanging the roles of $\boldsymbol{u}$ and $\boldsymbol{v}$ in (2.3), we find that

$$
\left\langle\gamma_{\mathbf{t}}(\boldsymbol{u}), \boldsymbol{\pi}_{\mathbf{t}}(\boldsymbol{v})\right\rangle_{\mathbf{t}, \Sigma}=-\left\langle\gamma_{\mathbf{t}}(\boldsymbol{v}), \boldsymbol{\pi}_{\mathbf{t}}(\boldsymbol{u})\right\rangle_{\mathbf{t}, \Sigma} \quad \forall \boldsymbol{u}, \boldsymbol{v} \in \mathbf{H}(\operatorname{curl} ; \Omega) .
$$

Copyright $@$ by SIAM. Unauthorized reproduction of this article is prohibited. 
3. The model problem. We consider a bounded, connected, and simply connected polyhedron $\Omega_{s} \subset \mathbb{R}^{3}$ representing a homogeneous elastic body immersed in an electromagnetic medium filling the whole space. We assume that the system consisting of the electromagnetic field and the elastic body interacts only through the interface $\Sigma:=\partial \Omega_{s}$.

Let $\epsilon, \mu$, and $\sigma$ be the electric permittivity, the magnetic permeability, and the conductivity of the medium, respectively. These coefficients are piecewise regular real valued scalar functions satisfying, in $\mathbb{R}^{3} \backslash \Omega_{s}$,

$$
\mu_{0} \leq \mu(\boldsymbol{x}) \leq \bar{\mu}, \quad \epsilon_{0} \leq \epsilon(\boldsymbol{x}) \leq \bar{\epsilon}, \quad \text { and } \quad 0 \leq \sigma(\boldsymbol{x}) \leq \bar{\sigma},
$$

where the constants $\epsilon_{0}$ and $\mu_{0}$ denote the electric permittivity and magnetic permeability of free space, respectively, and $\bar{\mu}, \bar{\epsilon}$, and $\bar{\sigma}$ are given upper bounds. Moreover, we assume that we have vacuum conditions sufficiently far from the obstacle; i.e., there exists $R>0$ such that

$$
\mu(\boldsymbol{x})=\mu_{0}, \quad \epsilon(\boldsymbol{x})=\epsilon_{0}, \quad \text { and } \quad \sigma(\boldsymbol{x})=0 \quad \forall \boldsymbol{x},|\boldsymbol{x}| \geq R .
$$

The incident electric and magnetic fields $\mathcal{E}^{i}$ and $\mathcal{H}^{i}$ are supposed to exhibit a timeharmonic behavior with frequency $\omega$ and complex amplitudes $\boldsymbol{E}^{i}$ and $\boldsymbol{H}^{i}$, respectively. Hence, the total electric and magnetic fields also have a time-harmonic behavior with frequency $\omega$, namely,

$$
\begin{aligned}
\mathcal{E}(\boldsymbol{x}, t) & =\operatorname{Re}\left\{\exp (-\imath \omega t) \epsilon_{0}^{-1 / 2} \boldsymbol{E}(\boldsymbol{x})\right\}, \\
\mathcal{H}(\boldsymbol{x}, t) & =\operatorname{Re}\left\{\exp (-\imath \omega t) \mu_{0}^{-1 / 2} \boldsymbol{H}(\boldsymbol{x})\right\},
\end{aligned}
$$

where the complex amplitudes $\boldsymbol{E}$ and $\boldsymbol{H}$ satisfy

$$
\begin{array}{ll}
\operatorname{curl}(\boldsymbol{E})-\imath k b \boldsymbol{H}=\mathbf{0} & \text { in } \mathbb{R}^{3} \backslash \Omega_{s}, \\
\operatorname{curl}(\boldsymbol{H})+\imath k a \boldsymbol{E}=\mathbf{0} & \text { in } \mathbb{R}^{3} \backslash \Omega_{s},
\end{array}
$$

$k:=\omega \sqrt{\epsilon_{0} \mu_{0}}$ is the wave number, and

$$
a(\boldsymbol{x}):=\frac{\epsilon(\boldsymbol{x})}{\epsilon_{0}}+\imath \frac{\sigma(\boldsymbol{x})}{\epsilon_{0} \omega} \quad \text { and } \quad b(\boldsymbol{x}):=\frac{\mu(\boldsymbol{x})}{\mu_{0}} \quad \forall \boldsymbol{x} \in \mathbb{R}^{3} .
$$

It is clear from (3.2) that

$$
a(\boldsymbol{x})=b(\boldsymbol{x})=1 \quad \forall \boldsymbol{x},|\boldsymbol{x}| \geq R .
$$

On the other hand, the solid is supposed to be isotropic and linearly elastic with mass density $\rho_{s}$ and Lamé constants $\mu_{s}$ and $\lambda_{s}$, which means, in particular, that the corresponding constitutive equation is given by

$$
\boldsymbol{\sigma}=\mathcal{C} \varepsilon(\boldsymbol{u}) \quad \text { in } \Omega_{s}
$$

where $\boldsymbol{\varepsilon}(\boldsymbol{u}):=\frac{1}{2}\left(\nabla \boldsymbol{u}+(\nabla \boldsymbol{u})^{\mathrm{t}}\right)$ is the strain tensor of small deformations, $\nabla$ is the gradient tensor, and $\mathcal{C}$ is the elasticity operator given by Hooke's law,

$$
\mathcal{C} \boldsymbol{\zeta}:=\lambda_{s} \operatorname{tr}(\boldsymbol{\zeta}) \mathbf{I}+2 \mu_{s} \boldsymbol{\zeta} \quad \forall \boldsymbol{\zeta} \in\left[L^{2}\left(\Omega_{s}\right)\right]^{3 \times 3} .
$$


Since the elastic displacement is also a time-harmonic field with the same frequency $\omega$, the unknowns $\boldsymbol{\sigma}$ and $\boldsymbol{u}$ satisfy the elastodynamic equation:

$$
\operatorname{div}(\boldsymbol{\sigma})+\kappa_{s}^{2} \boldsymbol{u}=\mathbf{0} \quad \text { in } \Omega_{s},
$$

where $\kappa_{s}:=\sqrt{\rho_{s}} \omega$ is the wave number in the obstacle.

We now let $\boldsymbol{n}$ denote the unit normal on $\Sigma$ oriented towards the exterior of $\Omega_{s}$. Then, according to Voigt's model (see [10], [20]), the transmission conditions coupling (3.3), (3.6), and (3.8) on $\Sigma$ are given by

$$
\begin{aligned}
\boldsymbol{E} \times \boldsymbol{n} & =\boldsymbol{u} \times \boldsymbol{n} & & \text { on } \Sigma, \\
\frac{\imath}{k} \boldsymbol{H} \times \boldsymbol{n} & =-\boldsymbol{\sigma} \boldsymbol{n} & & \text { on } \Sigma .
\end{aligned}
$$

In addition, the scattered electromagnetic field exhibits the Silver-Muller asymptotic behavior

$$
\left(\boldsymbol{E}-\boldsymbol{E}^{i}\right) \times \frac{\boldsymbol{x}}{|\boldsymbol{x}|}+\left(\boldsymbol{H}-\boldsymbol{H}^{i}\right)=o\left(\frac{1}{|\boldsymbol{x}|}\right)
$$

as $|\boldsymbol{x}| \rightarrow+\infty$, uniformly for all directions $\frac{\boldsymbol{x}}{|\boldsymbol{x}|}$. We notice that this asymptotic behavior implies that the outgoing waves are absorbed by the far field. Motivated by this fact, and aiming to obtain a suitable simplification of our model problem, we now introduce a sufficiently large sphere $\Gamma$ centered at the origin, define $\Omega_{m}$ as the annular region bounded by $\Sigma$ and $\Gamma$, and consider the boundary condition:

$$
\left(\boldsymbol{E}-\boldsymbol{E}^{i}\right) \times \boldsymbol{n}+\left(\boldsymbol{H}-\boldsymbol{H}^{i}\right)=\mathbf{0} \quad \text { on } \Gamma,
$$

where $\boldsymbol{n}$ denotes also the unit outward normal on $\Gamma$. Actually, in order to avoid later introducing a nonconforming Galerkin scheme, we may simply think of $\Gamma$ as the polyhedral surface resulting from a sufficiently accurate approximation of the given sphere.

In this way, (3.3), (3.6), (3.8), (3.9), (3.11), the expression $\boldsymbol{E}=-(\imath k a)^{-1}$ $\operatorname{curl}(\boldsymbol{H})$ of the electric field in terms of $\boldsymbol{H}$, and the fact that $a \equiv 1$ on $\Gamma$ (cf. (3.5)) lead us to the following formulation of the problem: Find $\boldsymbol{H}: \Omega_{m} \rightarrow \mathbb{C}^{3}, \boldsymbol{\sigma}: \Omega_{s} \rightarrow \mathbb{C}^{3 \times 3}$, and $\boldsymbol{u}: \Omega_{s} \rightarrow \mathbb{C}^{3}$ such that

$$
\begin{aligned}
\operatorname{curl}\left(a^{-1} \operatorname{curl}(\boldsymbol{H})\right)-k^{2} b \boldsymbol{H} & =\mathbf{0} & & \text { in } \Omega_{m}, \\
\boldsymbol{\sigma} & =\mathcal{C} \varepsilon(\boldsymbol{u}) & & \text { in } \Omega_{s}, \\
\operatorname{div}(\boldsymbol{\sigma})+\kappa_{s}^{2} \boldsymbol{u} & =\mathbf{0} & & \text { in } \Omega_{s}, \\
a^{-1} \operatorname{curl}(\boldsymbol{H}) \times \boldsymbol{n}+\imath k \boldsymbol{u} \times \boldsymbol{n} & =\mathbf{0} & & \text { on } \Sigma, \\
k^{2} \boldsymbol{\sigma} \boldsymbol{n}+\imath k \boldsymbol{H} \times \boldsymbol{n} & =\mathbf{0} & & \text { on } \Sigma, \\
\operatorname{curl}(\boldsymbol{H}) \times \boldsymbol{n}-\imath k \boldsymbol{H} & =\mathbf{g} & & \text { on } \Gamma,
\end{aligned}
$$

where $\mathbf{g}:=-\imath k\left(\boldsymbol{E}^{i} \times \boldsymbol{n}+\boldsymbol{H}^{i}\right)$. Note here that the transmission conditions on $\Sigma$ and the boundary condition on $\Gamma$ can be expressed in terms of the tangential and normal trace mappings $\gamma_{\mathbf{t}}$ and $\gamma_{\boldsymbol{n}}$, as follows:

$$
\begin{gathered}
\boldsymbol{\gamma}_{\mathbf{t}}\left(a^{-1} \operatorname{curl}(\boldsymbol{H})\right)=-\imath k \boldsymbol{\gamma}_{\mathbf{t}}(\boldsymbol{u}) \quad \text { on } \Sigma, \\
\imath k \boldsymbol{\gamma}_{\mathbf{t}}(\boldsymbol{H})=-k^{2} \boldsymbol{\gamma}_{\boldsymbol{n}}(\boldsymbol{\sigma}) \quad \text { on } \Sigma
\end{gathered}
$$

and

$$
\gamma_{\mathbf{t}}(\operatorname{curl}(\boldsymbol{H}))=\imath k \boldsymbol{H}+\mathbf{g} \quad \text { on } \Gamma .
$$


4. The continuous variational formulation. In this section we derive the full continuous variational formulation of (3.12). We begin by noticing, as we will see below, that the natural space for the magnetic field is given by

$$
\mathbf{H}_{\Gamma}\left(\operatorname{curl} ; \Omega_{m}\right):=\left\{\boldsymbol{W} \in \mathbf{H}\left(\operatorname{curl} ; \Omega_{m}\right): \quad \boldsymbol{\pi}_{\mathbf{t}}(\boldsymbol{W}) \in \mathbf{L}_{\mathbf{t}}^{2}(\Gamma)\right\},
$$

which, equipped with the graph norm

$$
\|\boldsymbol{W}\|_{\mathbf{H}_{\Gamma}\left(\mathbf{c u r l} ; \Omega_{m}\right)}^{2}:=\|\boldsymbol{W}\|_{\mathbf{H}\left(\operatorname{curl} ; \Omega_{m}\right)}^{2}+\left\|\boldsymbol{\pi}_{\mathbf{t}}(\boldsymbol{W})\right\|_{\left[L^{2}(\Gamma)\right]^{3}}^{2}
$$

is a Hilbert space.

Now, we test the first equation of (3.12) with a function $\boldsymbol{W} \in \mathbf{H}_{\Gamma}\left(\mathbf{c u r l} ; \Omega_{m}\right)$, and use Green's formula (2.3) and the fact that $a \equiv 1$ on $\Gamma$ (cf. (3.5)) to obtain

$$
\begin{aligned}
\int_{\Omega_{m}} & \left\{a^{-1} \operatorname{curl}(\boldsymbol{H}) \cdot \operatorname{curl}(\boldsymbol{W})-k^{2} b \boldsymbol{H} \cdot \boldsymbol{W}\right\} \\
& +\left\langle\boldsymbol{\gamma}_{\mathbf{t}}\left(a^{-1} \operatorname{curl}(\boldsymbol{H})\right), \boldsymbol{\pi}_{\mathbf{t}}(\boldsymbol{W})\right\rangle_{\mathbf{t}, \Sigma}-\left\langle\boldsymbol{\gamma}_{\mathbf{t}}(\operatorname{curl}(\boldsymbol{H})), \boldsymbol{\pi}_{\mathbf{t}}(\boldsymbol{W})\right\rangle_{\mathbf{t}, \Gamma}=0 .
\end{aligned}
$$

Then, incorporating the transmission condition (3.13) and the boundary condition (3.15) and using the identity (2.4), we find that (4.2) becomes

$$
\mathbf{a}_{m}(\boldsymbol{H}, \boldsymbol{W})+\imath k\left\langle\gamma_{\mathbf{t}}(\boldsymbol{W}), \boldsymbol{\pi}_{\mathbf{t}}(\boldsymbol{u})\right\rangle_{\mathbf{t}, \Sigma}=\int_{\Gamma} \mathbf{g} \cdot \boldsymbol{\pi}_{\mathbf{t}}(\boldsymbol{W}) \quad \forall \boldsymbol{W} \in \mathbf{H}_{\Gamma}\left(\operatorname{curl} ; \Omega_{m}\right),
$$

where $\mathbf{a}_{m}: \mathbf{H}_{\Gamma}\left(\mathbf{c u r l} ; \Omega_{m}\right) \times \mathbf{H}_{\Gamma}\left(\mathbf{c u r l} ; \Omega_{m}\right) \rightarrow \mathbb{C}$ is the bounded bilinear form defined by

$$
\begin{aligned}
& \mathbf{a}_{m}(\boldsymbol{H}, \boldsymbol{W}):=\int_{\Omega_{m}}\left\{a^{-1} \operatorname{curl}(\boldsymbol{H}) \cdot \operatorname{curl}(\boldsymbol{W})-k^{2} b \boldsymbol{H} \cdot \boldsymbol{W}\right\} \\
&-\imath k \int_{\Gamma} \boldsymbol{\pi}_{\mathbf{t}}(\boldsymbol{H}) \cdot \boldsymbol{\pi}_{\mathbf{t}}(\boldsymbol{W}) \quad \forall \boldsymbol{H}, \boldsymbol{W} \in \mathbf{H}_{\Gamma}\left(\operatorname{curl} ; \Omega_{m}\right) .
\end{aligned}
$$

On the other hand, in the obstacle $\Omega_{s}$ we proceed as in [12] and introduce the antisymmetric part $\boldsymbol{r}:=\nabla \boldsymbol{u}-\boldsymbol{\varepsilon}(\boldsymbol{u})$ of the tensor $\nabla \boldsymbol{u}$ and the trace $\boldsymbol{\psi}:=\gamma(\boldsymbol{u})$ on $\Sigma$ as additional unknowns. Then, we multiply the constitutive law (cf. (3.6)) $\mathcal{C}^{-1} \boldsymbol{\sigma}=\boldsymbol{\varepsilon}(\boldsymbol{u})=\nabla \boldsymbol{u}-\boldsymbol{r}$ by a test function $\boldsymbol{\tau} \in \mathbf{H}\left(\mathbf{d i v} ; \Omega_{s}\right)$ and integrate by parts to obtain

$$
\int_{\Omega_{s}} \mathcal{C}^{-1} \boldsymbol{\sigma}: \boldsymbol{\tau}=-\int_{\Omega_{s}} \boldsymbol{u} \cdot \operatorname{div}(\boldsymbol{\tau})+\left\langle\gamma_{\boldsymbol{n}}(\boldsymbol{\tau}), \boldsymbol{\psi}\right\rangle_{\Sigma}-\int_{\Omega_{s}} \boldsymbol{r}: \boldsymbol{\tau} \quad \forall \boldsymbol{\tau} \in \mathbf{H}\left(\operatorname{div} ; \Omega_{s}\right),
$$

where, hereafter, $\langle\cdot, \cdot\rangle_{\Sigma}$ stands for the duality pairing between the spaces $\left[H^{-1 / 2}(\Sigma)\right]^{3}$ and $\left[H^{1 / 2}(\Sigma)\right]^{3}$ with respect to the $\left[L^{2}(\Sigma)\right]^{3}$-inner product. Next, the displacement field $\boldsymbol{u}$ is eliminated from the last identity by using the expression

$$
\boldsymbol{u}=-\frac{1}{\kappa_{s}^{2}} \operatorname{div}(\boldsymbol{\sigma}) \quad \text { in } \Omega_{s},
$$

which follows from (3.8). In this way, we arrive at the following variational formulation in $\Omega_{s}$ :

$$
\mathbf{a}_{s}(\boldsymbol{\sigma}, \boldsymbol{\tau})-k^{2} \int_{\Omega_{s}} \boldsymbol{r}: \boldsymbol{\tau}+k^{2}\left\langle\gamma_{\boldsymbol{n}}(\boldsymbol{\tau}), \boldsymbol{\psi}\right\rangle_{\Sigma}=0 \quad \forall \boldsymbol{\tau} \in \mathbf{H}\left(\operatorname{div} ; \Omega_{s}\right)
$$


where $\mathbf{a}_{s}: \mathbf{H}\left(\operatorname{div} ; \Omega_{s}\right) \times \mathbf{H}\left(\operatorname{div} ; \Omega_{s}\right) \rightarrow \mathbb{C}$ is the bounded bilinear form defined by

$$
\mathbf{a}_{s}(\boldsymbol{\sigma}, \boldsymbol{\tau}):=k^{2}\left\{-\int_{\Omega_{s}} \mathcal{C}^{-1} \boldsymbol{\sigma}: \boldsymbol{\tau}+\frac{1}{\kappa_{s}^{2}} \int_{\Omega_{s}} \operatorname{div}(\boldsymbol{\sigma}) \cdot \operatorname{div}(\boldsymbol{\tau})\right\}
$$

for all $\boldsymbol{\sigma}, \boldsymbol{\tau} \in \mathbf{H}\left(\operatorname{div} ; \Omega_{s}\right)$. Finally, the symmetry of the stress tensor $\boldsymbol{\sigma}$ and the second transmission condition on $\Sigma$ (cf. (3.14)) are imposed weakly through the equations

$$
k^{2} \int_{\Omega_{s}} \boldsymbol{\sigma}: s=0 \quad \forall s \in\left[L^{2}\left(\Omega_{s}\right)\right]_{\mathrm{asym}}^{3 \times 3}
$$

and

$$
k^{2}\left\langle\boldsymbol{\gamma}_{\boldsymbol{n}}(\boldsymbol{\sigma}), \boldsymbol{\varphi}\right\rangle_{\Sigma}+\imath k\left\langle\boldsymbol{\gamma}_{\mathbf{t}}(\boldsymbol{H}), \boldsymbol{\pi}_{\mathbf{t}}(\boldsymbol{\varphi})\right\rangle_{\mathbf{t}, \Sigma}=0 \quad \forall \boldsymbol{\varphi} \in\left[H^{1 / 2}(\Sigma)\right]^{3},
$$

respectively, where

$$
\left[L^{2}\left(\Omega_{s}\right)\right]_{\mathrm{asym}}^{3 \times 3}:=\left\{\boldsymbol{s} \in\left[L^{2}\left(\Omega_{s}\right)\right]^{3 \times 3}: \quad \boldsymbol{s}=-\boldsymbol{s}^{\mathrm{t}}\right\} .
$$

We now introduce the spaces

$$
\mathbb{X}:=\mathbf{H}_{\Gamma}\left(\operatorname{curl} ; \Omega_{m}\right) \times \mathbf{H}\left(\operatorname{div} ; \Omega_{s}\right) \quad \text { and } \quad \mathbb{M}:=\left[H^{1 / 2}(\Sigma)\right]^{3} \times\left[L^{2}\left(\Omega_{s}\right)\right]_{\mathrm{asym}}^{3 \times 3}
$$

endowed with the corresponding Hilbertian product norms. Then, (4.3), (4.6), (4.8), and (4.9) yield the following global variational formulation of problem (3.12): Find $(\boldsymbol{H}, \boldsymbol{\sigma}) \in \mathbb{X}$ and $(\boldsymbol{\psi}, \boldsymbol{r}) \in \mathbb{M}$ such that

$$
\begin{array}{rlrl}
\mathbf{A}((\boldsymbol{H}, \boldsymbol{\sigma}),(\boldsymbol{W}, \boldsymbol{\tau}))+\mathbf{B}((\boldsymbol{W}, \boldsymbol{\tau}),(\boldsymbol{\psi}, \boldsymbol{r}))=\mathbf{L}((\boldsymbol{W}, \boldsymbol{\tau})) & & \forall(\boldsymbol{W}, \boldsymbol{\tau}) \in \mathbb{X}, \\
\mathbf{B}((\boldsymbol{H}, \boldsymbol{\sigma}),(\boldsymbol{\varphi}, \boldsymbol{s})) & =0 & & \forall(\boldsymbol{\varphi}, \boldsymbol{s}) \in \mathbb{M},
\end{array}
$$

where $\mathbf{A}: \mathbb{X} \times \mathbb{X} \rightarrow \mathbb{C}$ and $\mathbf{B}: \mathbb{X} \times \mathbb{M} \rightarrow \mathbb{C}$ are the bounded bilinear forms defined by

$$
\mathbf{A}((\boldsymbol{H}, \boldsymbol{\sigma}),(\boldsymbol{W}, \boldsymbol{\tau})):=\mathbf{a}_{m}(\boldsymbol{H}, \boldsymbol{W})+\mathbf{a}_{s}(\boldsymbol{\sigma}, \boldsymbol{\tau})
$$

for all $(\boldsymbol{H}, \boldsymbol{\sigma}),(\boldsymbol{W}, \boldsymbol{\tau}) \in \mathbb{X}$, and

$$
\mathbf{B}((\boldsymbol{W}, \boldsymbol{\tau}),(\boldsymbol{\varphi}, \boldsymbol{s})):=-k^{2} \int_{\Omega_{s}} \boldsymbol{\tau}: s+k^{2}\left\langle\boldsymbol{\gamma}_{\boldsymbol{n}}(\boldsymbol{\tau}), \boldsymbol{\varphi}\right\rangle_{\Sigma}+\imath k\left\langle\boldsymbol{\gamma}_{\mathbf{t}}(\boldsymbol{W}), \boldsymbol{\pi}_{\mathbf{t}}(\boldsymbol{\varphi})\right\rangle_{\mathbf{t}, \Sigma}
$$

for all $((\boldsymbol{W}, \boldsymbol{\tau}),(\boldsymbol{\varphi}, \boldsymbol{s})) \in \mathbb{X} \times \mathbb{M}$, and where $\mathbf{L}: \mathbb{X} \rightarrow \mathbb{C}$ is the bounded linear form given by

$$
\mathbf{L}((\boldsymbol{W}, \boldsymbol{\tau})):=\int_{\Gamma} \mathbf{g} \cdot \boldsymbol{\pi}_{\mathbf{t}}(\boldsymbol{W}) .
$$

5. Analysis of the continuous variational formulation. In this section we proceed analogously to [7] (see also [12]) and employ suitable decompositions of $\mathbf{H}_{\Gamma}\left(\mathbf{c u r l} ; \Omega_{m}\right)$ and $\mathbf{H}\left(\mathbf{d i v} ; \Omega_{s}\right)$ to prove that (4.10) becomes a compact perturbation of a well-posed problem. In particular, the splitting of $\mathbf{H}\left(\mathbf{d i v} ; \Omega_{s}\right)$ is defined in terms of an elasticity problem in $\Omega_{s}$ with Neumann boundary conditions, whereas a well-known result on divergence-free potential vectors is the basis of the splitting of $\mathbf{H}_{\Gamma}\left(\mathbf{c u r l} ; \Omega_{m}\right)$. More precisely, let us first introduce the space

$$
\mathbf{H}\left(\operatorname{div} 0 ; \Omega_{m}\right):=\left\{\boldsymbol{W} \in \mathbf{H}\left(\operatorname{div} ; \Omega_{m}\right): \operatorname{div}(\boldsymbol{W})=0 \text { in } \Omega_{m} \text { and }\left\langle\boldsymbol{\gamma}_{\boldsymbol{n}}(\boldsymbol{W}), 1\right\rangle_{\Sigma}=0\right\} \text {. }
$$

Then, we recall from [15] the following classical result. 
Lemma 5.1. There exists a bounded linear operator

$$
\mathcal{L}: \mathbf{H}\left(\operatorname{div} 0 ; \Omega_{m}\right) \rightarrow\left[H^{1}\left(\Omega_{m}\right)\right]^{3}
$$

such that $\operatorname{div}(\mathcal{L}(\boldsymbol{W}))=0$ and $\operatorname{curl}(\mathcal{L}(\boldsymbol{W}))=\boldsymbol{W}$ for all $\boldsymbol{W} \in \mathbf{H}\left(\operatorname{div} 0 ; \Omega_{m}\right)$.

Proof. For the proof, see Theorem 3.4 in Chapter I of [15] or Lemma 3.5 in [2].

5.1. A regular splitting of $\mathbf{H}_{\boldsymbol{\Gamma}}\left(\operatorname{curl} ; \boldsymbol{\Omega}_{\boldsymbol{m}}\right)$. We first observe that, given $\boldsymbol{W} \in$ $\mathbf{H}_{\Gamma}\left(\operatorname{curl} ; \Omega_{m}\right), \boldsymbol{c u r l}(\boldsymbol{W})$ belongs to $\mathbf{H}\left(\operatorname{div} 0 ; \Omega_{m}\right)$. In fact, it is clear that $\operatorname{curl}(\boldsymbol{W})$ is a divergence-free element of $\mathbf{H}\left(\operatorname{div} ; \Omega_{m}\right)$. Next, in order to show that $\left\langle\gamma_{\boldsymbol{n}}(\boldsymbol{\operatorname { c u r l }}(\boldsymbol{W})), 1\right\rangle_{\Sigma}$ $=0$ we recall that $\gamma_{\boldsymbol{n}}(\boldsymbol{\operatorname { c u r l }}(\boldsymbol{W}))=\operatorname{div}_{\Sigma}\left(\boldsymbol{\gamma}_{\mathbf{t}}(\boldsymbol{W})\right)$ and that the adjoint of $\operatorname{div}_{\Sigma}$ is $-\nabla_{\Sigma}$ (see [5], [7]). It follows that

$$
\left\langle\gamma_{\boldsymbol{n}}(\operatorname{curl}(\boldsymbol{W})), 1\right\rangle_{\Sigma}=\left\langle\operatorname{div}_{\Sigma}\left(\boldsymbol{\gamma}_{\mathbf{t}}(\boldsymbol{W})\right), 1\right\rangle_{\Sigma}=-\left\langle\boldsymbol{\gamma}_{\mathbf{t}}(\boldsymbol{W}), \nabla_{\Sigma}(1)\right\rangle_{\mathbf{t}, \Sigma}=0 .
$$

Then, it is clear that the mapping $\operatorname{curl}: \mathbf{H}_{\Gamma}\left(\mathbf{c u r l} ; \Omega_{m}\right) \rightarrow \mathbf{H}\left(\operatorname{div} 0 ; \Omega_{m}\right)$ is bounded. In addition, it is easy to see, using the trace theorem, that the injection $\mathbf{i}:\left[H^{1}\left(\Omega_{m}\right)\right]^{3}$ $\hookrightarrow \mathbf{H}_{\Gamma}\left(\mathbf{c u r l} ; \Omega_{m}\right)$ is also bounded. Hence, we can introduce the bounded linear operator $\mathcal{P}_{m}:=\mathbf{i} \circ \mathcal{L} \circ$ curl; that is,

$$
\begin{aligned}
\mathcal{P}_{m}: \mathbf{H}_{\Gamma}\left(\operatorname{curl} ; \Omega_{m}\right) & \rightarrow \mathbf{H}_{\Gamma}\left(\operatorname{curl} ; \Omega_{m}\right), \\
\boldsymbol{W} & \mapsto \mathcal{P}_{m}(\boldsymbol{W}):=\mathcal{L}(\operatorname{curl}(\boldsymbol{W})) .
\end{aligned}
$$

Now, from Lemma 5.1 we have that $\operatorname{curl}\left(\mathcal{P}_{m}(\boldsymbol{W})\right)=\operatorname{curl}(\boldsymbol{W})$ in $\Omega_{m}$, which implies that $\mathcal{P}_{m}^{2}=\mathcal{P}_{m}$, and therefore $\mathcal{P}_{m}$ provides a stable and direct Helmholtz-type decomposition

$$
\mathbf{H}_{\Gamma}\left(\operatorname{curl} ; \Omega_{m}\right)=\mathcal{P}_{m}\left(\mathbf{H}_{\Gamma}\left(\operatorname{curl} ; \Omega_{m}\right)\right) \oplus\left(\mathcal{I}-\mathcal{P}_{m}\right)\left(\mathbf{H}_{\Gamma}\left(\operatorname{curl} ; \Omega_{m}\right)\right) .
$$

Hereafter, $\mathcal{I}$ stands for a generic identity operator. Equation (5.2) means that any element $\boldsymbol{H} \in \mathbf{H}_{\Gamma}\left(\mathbf{c u r l} ; \Omega_{m}\right)$ admits the unique regular splitting (cf. [4], [16])

$$
\boldsymbol{H}=\mathcal{P}_{m}(\boldsymbol{H})+\left(\mathcal{I}-\mathcal{P}_{m}\right)(\boldsymbol{H})
$$

and the norm

$\boldsymbol{W} \rightarrow \| \boldsymbol{W}||_{\mathbf{H}_{\Gamma}\left(\mathbf{c u r l} ; \Omega_{m}\right)}:=\left\{\left\|\mathcal{P}_{m}(\boldsymbol{W})\right\|_{\mathbf{H}_{\Gamma}\left(\mathbf{c u r l} ; \Omega_{m}\right)}^{2}+\left\|\left(\mathcal{I}-\mathcal{P}_{m}\right)(\boldsymbol{W})\right\|_{\mathbf{H}_{\Gamma}\left(\mathbf{c u r l} ; \Omega_{m}\right)}^{2}\right\}^{1 / 2}$

is equivalent to $\boldsymbol{W} \rightarrow\|\boldsymbol{W}\|_{\mathbf{H}_{\Gamma}\left(\mathbf{c u r l} ; \Omega_{m}\right)}$ on $\mathbf{H}_{\Gamma}\left(\mathbf{c u r l} ; \Omega_{m}\right)$. More precisely, since $\left\|\mathcal{P}_{m}\right\|=\left\|\mathcal{I}-\mathcal{P}_{m}\right\|$ (see Lemma 5 of $[23]$ ), we have

$$
\frac{1}{\sqrt{2}\left\|\mathcal{P}_{m}\right\|}\left\|\boldsymbol{W}\left|\left\|_{\mathbf{H}_{\Gamma}\left(\mathbf{c u r l} ; \Omega_{m}\right)} \leq\right\| \boldsymbol{W}\left\|_{\mathbf{H}_{\Gamma}\left(\operatorname{curl} ; \Omega_{m}\right)} \leq \sqrt{2}\right\| \boldsymbol{W}\right|\right\|_{\mathbf{H}_{\Gamma}\left(\operatorname{curl} ; \Omega_{m}\right)}
$$

for all $\boldsymbol{W} \in \mathbf{H}_{\Gamma}\left(\operatorname{curl} ; \Omega_{m}\right)$.

Finally, thanks to the compact imbedding $\left[H^{1}\left(\Omega_{m}\right)\right]^{3} \subset\left[L^{2}\left(\Omega_{m}\right)\right]^{3}$, we have the following result.

LEMma 5.2. The mapping $\mathcal{P}_{m}: \mathbf{H}_{\Gamma}\left(\mathbf{c u r l} ; \Omega_{m}\right) \rightarrow\left[L^{2}\left(\Omega_{m}\right)\right]^{3}$ is compact. 
5.2. A regular splitting of $\mathbf{H}\left(\operatorname{div} ; \boldsymbol{\Omega}_{\boldsymbol{s}}\right)$. Here we extend the analysis of section 4.1 in [12] to the $3 \mathrm{D}$ case. In fact, let $\mathbb{R M}\left(\Omega_{s}\right)$ be the space of rigid body motions in $\Omega_{s}$; that is,

$$
\mathbb{R M}\left(\Omega_{s}\right):=\left\{\boldsymbol{v}: \Omega_{s} \rightarrow \mathbb{C}^{3}: \quad \boldsymbol{v}(\boldsymbol{x})=\boldsymbol{\alpha}+\boldsymbol{\beta} \times \boldsymbol{x} \quad \forall \boldsymbol{x} \in \Omega_{s}, \boldsymbol{\alpha}, \boldsymbol{\beta} \in \mathbb{C}^{3}\right\},
$$

and let $\mathbf{M}:\left[L^{2}\left(\Omega_{s}\right)\right]^{3} \rightarrow \mathbb{R M}\left(\Omega_{s}\right)$ be the $\left[L^{2}\left(\Omega_{s}\right)\right]^{3}$-orthogonal projection. Then, given $\boldsymbol{\tau} \in \mathbf{H}\left(\operatorname{div} ; \Omega_{s}\right)$, we let $\tilde{\boldsymbol{u}} \in\left[H^{1}\left(\Omega_{s}\right)\right]^{3}$ be the unique (up to an element in $\mathbb{R M}\left(\Omega_{s}\right)$ ) solution of the boundary value problem

$$
\tilde{\boldsymbol{\sigma}}=\mathcal{C} \varepsilon(\tilde{\boldsymbol{u}}), \quad \operatorname{div}(\tilde{\boldsymbol{\sigma}})=(\mathcal{I}-\mathbf{M})(\operatorname{div}(\boldsymbol{\tau})) \quad \text { in } \Omega_{s}, \quad \boldsymbol{\gamma}_{\boldsymbol{n}}(\tilde{\boldsymbol{\sigma}})=\mathbf{0} \quad \text { on } \Sigma .
$$

Owing to the regularity result for the Neumann-elasticity problem on Lipschitz polyhedral domains (see [11]), we know that there exists $\epsilon \in(0,1]$ such that the solution $\tilde{\boldsymbol{u}}$ of $(5.6)$ belongs to $\left[H^{1+\epsilon}\left(\Omega_{s}\right)\right]^{3}$ and satisfies

$$
\|\tilde{\boldsymbol{u}}\|_{\left[H^{1+\epsilon}\left(\Omega_{s}\right)\right]^{3}} \leq C\|\operatorname{div}(\boldsymbol{\tau})\|_{\left[L^{2}\left(\Omega_{s}\right)\right]^{3}} .
$$

On the other hand, following the usual procedure, we deduce that the dual-mixed variational formulation of $(5.6)$ reads: Find $(\tilde{\boldsymbol{\sigma}},(\tilde{\boldsymbol{u}}, \tilde{\boldsymbol{r}})) \in \mathbb{H} \times \mathbb{Q}$ such that

$$
\begin{aligned}
\int_{\Omega_{s}} \mathcal{C}^{-1} \tilde{\boldsymbol{\sigma}}: \tilde{\boldsymbol{\tau}}+ & \int_{\Omega_{s}}\{\tilde{\boldsymbol{u}} \cdot \operatorname{div}(\tilde{\boldsymbol{\tau}})+\tilde{\boldsymbol{r}}: \tilde{\boldsymbol{\tau}}\} \\
& =0, \\
\int_{\Omega_{s}}\{\tilde{\boldsymbol{v}} \cdot \operatorname{div}(\tilde{\boldsymbol{\sigma}})+\tilde{\boldsymbol{s}}: \tilde{\boldsymbol{\sigma}}\} & =\int_{\Omega_{s}} \tilde{\boldsymbol{v}} \cdot(\mathcal{I}-\mathbf{M})(\operatorname{div}(\boldsymbol{\tau}))
\end{aligned}
$$

for all $(\tilde{\boldsymbol{\tau}},(\tilde{\boldsymbol{v}}, \tilde{\boldsymbol{s}})) \in \mathbb{H} \times \mathbb{Q}$, where $\tilde{\boldsymbol{r}}$ is the auxiliary unknown (named rotation) given by

$$
\begin{gathered}
\tilde{\boldsymbol{r}}:=\frac{1}{2}\left(\nabla \tilde{\boldsymbol{u}}-(\nabla \tilde{\boldsymbol{u}})^{\mathrm{t}}\right), \\
\mathbb{H}:=\left\{\boldsymbol{\tau} \in \mathbf{H}\left(\operatorname{div} ; \Omega_{s}\right): \quad \gamma_{\boldsymbol{n}}(\boldsymbol{\tau})=\mathbf{0} \quad \text { on } \Sigma\right\},
\end{gathered}
$$

and

$$
\mathbb{Q}:=(\mathcal{I}-\mathbf{M})\left(\left[L^{2}\left(\Omega_{s}\right)\right]^{3}\right) \times\left[L^{2}\left(\Omega_{s}\right)\right]_{\mathrm{asym}}^{3 \times 3} .
$$

Then, adapting the theory from [3], [19], one can easily show that (5.8) is well-posed. Moreover, using (5.7), we deduce that

$$
\|\tilde{\boldsymbol{\sigma}}\|_{\left[H^{\epsilon}\left(\Omega_{s}\right)\right]^{3 \times 3}}+\|\tilde{\boldsymbol{u}}\|_{\left[H^{1+\epsilon}\left(\Omega_{s}\right)\right]^{3}}+\|\tilde{\boldsymbol{r}}\|_{\left[H^{\epsilon}\left(\Omega_{s}\right)\right]^{3 \times 3}} \leq C\|\operatorname{div}(\boldsymbol{\tau})\|_{\left[L^{2}\left(\Omega_{s}\right)\right]^{3}} .
$$

Then, we introduce the linear operator

$$
\begin{aligned}
\mathcal{P}_{s}: \mathbf{H}\left(\operatorname{div} ; \Omega_{s}\right) & \rightarrow \mathbf{H}\left(\operatorname{div} ; \Omega_{s}\right), \\
\boldsymbol{\tau} & \rightarrow \mathcal{P}_{s}(\boldsymbol{\tau}):=\tilde{\boldsymbol{\sigma}}
\end{aligned}
$$

The continuous dependence result for (5.8) insures that $\mathcal{P}_{s}$ is bounded. In addition, from the second equation of (5.8) we find that $\mathcal{P}_{s}(\boldsymbol{\tau})$ is a symmetric tensor and that $\operatorname{div}\left(\mathcal{P}_{s}(\boldsymbol{\tau})\right)=(\mathcal{I}-\mathbf{M})(\operatorname{div}(\boldsymbol{\tau}))$ in $\Omega_{s}$. The latter implies that $\mathcal{P}_{s}^{2}=\mathcal{P}_{s}$, and hence the following stable splitting holds:

$$
\mathbf{H}\left(\operatorname{div} ; \Omega_{s}\right)=\mathcal{P}_{s}\left(\mathbf{H}\left(\operatorname{div} ; \Omega_{s}\right)\right) \oplus\left(\mathcal{I}-\mathcal{P}_{s}\right)\left(\mathbf{H}\left(\operatorname{div} ; \Omega_{s}\right)\right) .
$$


This means that each tensor $\boldsymbol{\tau} \in \mathbf{H}\left(\mathbf{d i v} ; \Omega_{s}\right)$ admits the unique regular decomposition (cf. [12])

$$
\boldsymbol{\tau}=\mathcal{P}_{s}(\boldsymbol{\tau})+\left(\mathcal{I}-\mathcal{P}_{s}\right)(\boldsymbol{\tau})
$$

Moreover, the identity $\left\|\mathcal{P}_{s}\right\|=\left\|\mathcal{I}-\mathcal{P}_{s}\right\|$ (see Lemma 5 of [23]) yields

$$
\frac{1}{\sqrt{2}\left\|\mathcal{P}_{s}\right\|}\left|\left\|\boldsymbol{\tau}\left|\left\|_{\mathbf{H}\left(\operatorname{div} ; \Omega_{s}\right)} \leq\right\| \boldsymbol{\tau}\left\|_{\mathbf{H}\left(\operatorname{div} ; \Omega_{s}\right)} \leq \sqrt{2}\right\|\right| \boldsymbol{\tau} \mid\right\|_{\mathbf{H}\left(\operatorname{div} ; \Omega_{s}\right)} \quad \forall \boldsymbol{\tau} \in \mathbf{H}\left(\operatorname{div} ; \Omega_{s}\right)\right.
$$

where

$$
\boldsymbol{\tau} \rightarrow|\|\boldsymbol{\tau}\||_{\mathbf{H}\left(\mathbf{d i v} ; \Omega_{s}\right)}:=\left\{\left\|\mathcal{P}_{s}(\boldsymbol{\tau})\right\|_{\mathbf{H}\left(\mathbf{d i v} ; \Omega_{s}\right)}^{2}+\left\|\left(\mathcal{I}-\mathcal{P}_{s}\right)(\boldsymbol{\tau})\right\|_{\mathbf{H}\left(\mathbf{d i v} ; \Omega_{s}\right)}^{2}\right\}^{1 / 2} .
$$

LEMmA 5.3. The mappings $\mathcal{P}_{s}: \mathbf{H}\left(\operatorname{div} ; \Omega_{s}\right) \rightarrow\left[L^{2}\left(\Omega_{s}\right)\right]^{3 \times 3}$ and $\operatorname{div}\left(\mathcal{I}-\mathcal{P}_{s}\right):$ $\mathbf{H}\left(\operatorname{div} ; \Omega_{s}\right) \rightarrow\left[L^{2}\left(\Omega_{s}\right)\right]^{3}$ are compact.

Proof. The first assertion of the lemma is a consequence of the regularity result $\mathcal{P}_{s}\left(\mathbf{H}\left(\operatorname{div} ; \Omega_{s}\right)\right) \subseteq\left[H^{\epsilon}\left(\Omega_{s}\right)\right]^{3 \times 3}$ and the compact embedding $H^{\epsilon}\left(\Omega_{s}\right) \hookrightarrow L^{2}\left(\Omega_{s}\right)$. The second follows from the fact that $\operatorname{div}\left(\mathcal{I}-\mathcal{P}_{s}\right)$ is a finite rank operator since $\operatorname{div}(\mathcal{I}-$ $\left.\mathcal{P}_{s}\right)(\boldsymbol{\tau})=\mathbf{M}(\operatorname{div}(\boldsymbol{\tau})) \in \mathbb{R M}\left(\Omega_{s}\right)$ for all $\boldsymbol{\tau} \in \mathbf{H}\left(\operatorname{div} ; \Omega_{s}\right)$.

5.3. Well-posedness of the continuous formulation. In this section we apply the stable decompositions (5.2) and (5.13) to reformulate (4.10) as a compact perturbation of a well-posed problem. To this end, we first introduce the bounded bilinear forms

$$
\begin{aligned}
& \mathbf{a}_{m}^{+}(\boldsymbol{H}, \boldsymbol{W}):=\int_{\Omega_{m}}\left\{a^{-1} \operatorname{curl}(\boldsymbol{H}) \cdot \operatorname{curl}(\boldsymbol{W})+k^{2} b \boldsymbol{H} \cdot \boldsymbol{W}\right\} \\
&-\imath k \int_{\Gamma} \boldsymbol{\pi}_{\mathbf{t}}(\boldsymbol{H}) \cdot \boldsymbol{\pi}_{\mathbf{t}}(\boldsymbol{W}) \quad \forall \boldsymbol{H}, \boldsymbol{W} \in \mathbf{H}_{\Gamma}\left(\operatorname{curl} ; \Omega_{m}\right)
\end{aligned}
$$

and

$$
\mathbf{a}_{s}^{+}(\boldsymbol{\sigma}, \boldsymbol{\tau}):=k^{2}\left\{\int_{\Omega_{s}} \mathcal{C}^{-1} \boldsymbol{\sigma}: \boldsymbol{\tau}+\frac{1}{\kappa_{s}^{2}} \int_{\Omega_{s}} \operatorname{div}(\boldsymbol{\sigma}) \cdot \operatorname{div}(\boldsymbol{\tau})\right\} \quad \forall \boldsymbol{\sigma}, \boldsymbol{\tau} \in \mathbf{H}\left(\operatorname{div} ; \Omega_{s}\right),
$$

which arise, respectively, from the forms $\mathbf{a}_{m}$ and $\mathbf{a}_{s}$ (cf. (4.4) and (4.7)) after performing a suitable change of sign in each. More precisely, note that

$$
\mathbf{a}_{m}^{+}(\boldsymbol{H}, \boldsymbol{W})=\mathbf{a}_{m}(\boldsymbol{H}, \boldsymbol{W})+2 k^{2} \int_{\Omega_{m}} b \boldsymbol{H} \cdot \boldsymbol{W}
$$

and

$$
\mathbf{a}_{s}^{+}(\boldsymbol{\sigma}, \boldsymbol{\tau})=\mathbf{a}_{s}(\boldsymbol{\sigma}, \boldsymbol{\tau})+2 k^{2} \int_{\Omega_{s}} \mathcal{C}^{-1} \boldsymbol{\sigma}: \boldsymbol{\tau} .
$$

Then, employing (5.3) for each $\boldsymbol{H}, \boldsymbol{W} \in \mathbf{H}_{\Gamma}\left(\mathbf{c u r l} ; \Omega_{m}\right.$ ) and (5.14) for each $\boldsymbol{\sigma}, \boldsymbol{\tau} \in$ $\mathbf{H}\left(\mathbf{d i v} ; \Omega_{s}\right)$, we deduce from (4.11), (5.18), and (5.19) that the bilinear form $\mathbf{A}$ can be decomposed as

$$
\mathbf{A}((\boldsymbol{H}, \boldsymbol{\sigma}),(\boldsymbol{W}, \boldsymbol{\tau}))=\mathbf{A}_{0}((\boldsymbol{H}, \boldsymbol{\sigma}),(\boldsymbol{W}, \boldsymbol{\tau}))+\mathbf{K}_{0}((\boldsymbol{H}, \boldsymbol{\sigma}),(\boldsymbol{W}, \boldsymbol{\tau})),
$$


where

$$
\begin{aligned}
\mathbf{A}_{0}((\boldsymbol{H}, \boldsymbol{\sigma}),(\boldsymbol{W}, \boldsymbol{\tau}))=\mathbf{a}_{m}^{+}\left(\mathcal{P}_{m}(\boldsymbol{H}), \mathcal{P}_{m}(\boldsymbol{W})\right)-\mathbf{a}_{m}^{+}\left(\left(\mathcal{I}-\mathcal{P}_{m}\right)(\boldsymbol{H}),\left(\mathcal{I}-\mathcal{P}_{m}\right)(\boldsymbol{W})\right) \\
\quad-2 \imath k \int_{\Gamma} \boldsymbol{\pi}_{\mathbf{t}}\left(\left(\mathcal{I}-\mathcal{P}_{m}\right)(\boldsymbol{H})\right) \cdot \boldsymbol{\pi}_{\mathbf{t}}\left(\left(\mathcal{I}-\mathcal{P}_{m}\right)(\boldsymbol{W})\right) \\
\quad+\mathbf{a}_{s}^{+}\left(\mathcal{P}_{s}(\boldsymbol{\sigma}), \mathcal{P}_{s}(\boldsymbol{\tau})\right)-\mathbf{a}_{s}^{+}\left(\left(\mathcal{I}-\mathcal{P}_{s}\right)(\boldsymbol{\sigma}),\left(\mathcal{I}-\mathcal{P}_{s}\right)(\boldsymbol{\tau})\right)
\end{aligned}
$$

and

$$
\begin{aligned}
& \mathbf{K}_{0}((\boldsymbol{H}, \boldsymbol{\sigma}),(\boldsymbol{W}, \boldsymbol{\tau}))=2 \mathbf{a}_{m}^{+}\left(\left(\mathcal{I}-\mathcal{P}_{m}\right)(\boldsymbol{H}),\left(\mathcal{I}-\mathcal{P}_{m}\right)(\boldsymbol{W})\right) \\
& \quad+\mathbf{a}_{m}^{+}\left(\mathcal{P}_{m}(\boldsymbol{H}),\left(\mathcal{I}-\mathcal{P}_{m}\right)(\boldsymbol{W})\right)+2 \imath k \int_{\Gamma} \boldsymbol{\pi}_{\mathbf{t}}\left(\left(\mathcal{I}-\mathcal{P}_{m}\right)(\boldsymbol{H})\right) \cdot \boldsymbol{\pi}_{\mathbf{t}}\left(\left(\mathcal{I}-\mathcal{P}_{m}\right)(\boldsymbol{W})\right) \\
& \quad+\mathbf{a}_{m}^{+}\left(\left(\mathcal{I}-\mathcal{P}_{m}\right)(\boldsymbol{H}), \mathcal{P}_{m}(\boldsymbol{W})\right)-2 k^{2} \int_{\Omega_{m}} b \boldsymbol{H} \cdot \boldsymbol{W} \\
& \quad+2 \mathbf{a}_{s}^{+}\left(\left(\mathcal{I}-\mathcal{P}_{s}\right)(\boldsymbol{\sigma}),\left(\mathcal{I}-\mathcal{P}_{s}\right)(\boldsymbol{\tau})\right)+\mathbf{a}_{s}^{+}\left(\mathcal{P}_{s}(\boldsymbol{\sigma}),\left(\mathcal{I}-\mathcal{P}_{s}\right)(\boldsymbol{\tau})\right) \\
& \quad+\mathbf{a}_{s}^{+}\left(\left(\mathcal{I}-\mathcal{P}_{s}\right)(\boldsymbol{\sigma}), \mathcal{P}_{s}(\boldsymbol{\tau})\right)-2 k^{2} \int_{\Omega_{s}} \mathcal{C}^{-1} \boldsymbol{\sigma}: \boldsymbol{\tau} .
\end{aligned}
$$

Similarly, it is clear from (4.12) that the bilinear form $\mathbf{B}$ can be decomposed as

$$
\mathbf{B}((\boldsymbol{W}, \boldsymbol{\tau}),(\boldsymbol{\varphi}, \boldsymbol{s}))=\mathbf{B}_{0}((\boldsymbol{W}, \boldsymbol{\tau}),(\boldsymbol{\varphi}, \boldsymbol{s}))+\mathbf{K}_{1}((\boldsymbol{W}, \boldsymbol{\tau}),(\boldsymbol{\varphi}, \boldsymbol{s})),
$$

where

$$
\mathbf{B}_{0}((\boldsymbol{W}, \boldsymbol{\tau}),(\boldsymbol{\varphi}, \boldsymbol{s})):=-k^{2} \int_{\Omega_{s}} \boldsymbol{\tau}: \boldsymbol{s}+k^{2}\left\langle\boldsymbol{\gamma}_{\boldsymbol{n}}(\boldsymbol{\tau}), \boldsymbol{\varphi}\right\rangle_{\Sigma}
$$

and

$$
\mathbf{K}_{1}((\boldsymbol{W}, \boldsymbol{\tau}),(\boldsymbol{\varphi}, \boldsymbol{s})):=\imath k\left\langle\boldsymbol{\gamma}_{\mathbf{t}}(\boldsymbol{W}), \boldsymbol{\pi}_{\mathbf{t}}(\boldsymbol{\varphi})\right\rangle_{\mathbf{t}, \Sigma} .
$$

Next, we let $\mathbb{A}_{0}, \mathbb{K}_{0}: \mathbb{X} \rightarrow \mathbb{X}$ and $\mathbb{B}_{0}, \mathbb{K}_{1}: \mathbb{X} \rightarrow \mathbb{M}$ be the linear and bounded operators induced by the corresponding bilinear forms, and let $\mathbb{B}_{0}^{*}, \mathbb{K}_{1}^{*}: \mathbb{M} \rightarrow \mathbb{X}$ be the associated adjoint operators. Then, the continuous variational formulation (4.10) can be rewritten as the following matrix operator equation: Find $((\boldsymbol{H}, \boldsymbol{\sigma}),(\boldsymbol{\psi}, \boldsymbol{r})) \in \mathbb{X} \times \mathbb{M}$ such that

$$
\left(\begin{array}{cc}
\mathbb{A}_{0} & \mathbb{B}_{0}^{*} \\
\mathbb{B}_{0} & \mathbf{0}
\end{array}\right)\left(\begin{array}{c}
(\boldsymbol{H}, \boldsymbol{\sigma}) \\
(\boldsymbol{\psi}, \boldsymbol{r})
\end{array}\right)+\left(\begin{array}{cc}
\mathbb{K}_{0} & \mathbb{K}_{1}^{*} \\
\mathbb{K}_{1} & \mathbf{0}
\end{array}\right)\left(\begin{array}{c}
(\boldsymbol{H}, \boldsymbol{\sigma}) \\
(\boldsymbol{\psi}, \boldsymbol{r})
\end{array}\right)=\left(\begin{array}{c}
\mathbb{L} \\
\mathbf{0}
\end{array}\right)
$$

where $\mathbb{L} \in \mathbb{X}$ is the Riesz representant of $\mathbf{L}$.

In what follows we prove that the matrix operators on the left-hand side of (5.26) are invertible and compact, respectively. For the invertibility we apply the BabuškaBrezzi theory and begin the corresponding analysis by establishing the inf-sup condition for the bilinear form $\mathbf{B}_{0}$.

Lemma 5.4. There exists $\beta>0$ such that

$$
\sup _{(\boldsymbol{w}, \boldsymbol{\tau}) \in \mathbb{X} \backslash\{\mathbf{0}\}} \frac{\left|\mathbf{B}_{0}((\boldsymbol{W}, \boldsymbol{\tau}),(\boldsymbol{\varphi}, \boldsymbol{s}))\right|}{\|(\boldsymbol{W}, \boldsymbol{\tau})\|_{\mathbb{X}}} \geq \beta\|(\boldsymbol{\varphi}, \boldsymbol{s})\|_{\mathbb{M}} \quad \forall(\boldsymbol{\varphi}, \boldsymbol{s}) \in \mathbb{M} .
$$

Copyright (c) by SIAM. Unauthorized reproduction of this article is prohibited. 
Proof. It is easy to see that

$$
\begin{aligned}
& \sup _{(\boldsymbol{W}, \boldsymbol{\tau}) \in \mathbb{X} \backslash\{\mathbf{0}\}} \frac{\left|\mathbf{B}_{0}((\boldsymbol{W}, \boldsymbol{\tau}),(\boldsymbol{\varphi}, \boldsymbol{s}))\right|}{\|(\boldsymbol{W}, \boldsymbol{\tau})\|_{\mathbb{X}}} \\
& \quad=\sup _{\boldsymbol{\tau} \in \mathbf{H}\left(\operatorname{div} ; \Omega_{s}\right) \backslash\{\mathbf{0}\}} \frac{\left|-k^{2} \int_{\Omega_{s}} \boldsymbol{\tau}: \boldsymbol{s}+k^{2}\left\langle\boldsymbol{\gamma}_{\boldsymbol{n}}(\boldsymbol{\tau}), \boldsymbol{\varphi}\right\rangle_{\Sigma}\right|}{\|\boldsymbol{\tau}\|_{\mathbf{H}\left(\operatorname{div} ; \Omega_{s}\right)}} .
\end{aligned}
$$

Thus, the rest of the proof reduces to deriving the corresponding lower bound for the latter supremum, which, as shown in what follows, proceeds analogously to the 2D case (cf. [12, Lemma 4.3]).

We first let $\boldsymbol{S}:\left[H^{-1 / 2}(\Sigma)\right]^{3} \rightarrow \mathbf{H}\left(\mathbf{d i v} ; \Omega_{s}\right)$ be the bounded linear operator defined by

$$
\boldsymbol{S}(\boldsymbol{\xi}):=\nabla \mathbf{z} \quad \forall \boldsymbol{\xi}:=\left(\xi_{1}, \xi_{2}, \xi_{3}\right)^{\mathrm{t}} \in\left[H^{-1 / 2}(\Sigma)\right]^{3},
$$

where $\mathbf{z} \in\left[H^{1}\left(\Omega_{s}\right)\right]^{3}$ is the unique weak solution, up to a constant vector in $\mathbb{C}^{3}$, of the boundary value problem with Neumann boundary conditions:

$$
\Delta \mathbf{z}=\frac{1}{\left|\Omega_{s}\right|}\left(\left\langle\xi_{1}, 1\right\rangle_{\Sigma},\left\langle\xi_{2}, 1\right\rangle_{\Sigma},\left\langle\xi_{3}, 1\right\rangle_{\Sigma}\right)^{\mathrm{t}} \quad \text { in } \Omega_{s}, \quad \gamma_{\boldsymbol{n}}(\nabla \mathbf{z})=\boldsymbol{\xi} \quad \text { on } \Sigma .
$$

It follows that $\gamma_{\boldsymbol{n}}(\boldsymbol{S}(\boldsymbol{\xi}))=\boldsymbol{\xi}$ on $\Sigma$, and hence

$$
\begin{aligned}
& \sup _{\boldsymbol{\tau} \in \mathbf{H}\left(\operatorname{div} ; \Omega_{s}\right) \backslash\{0\}} \frac{\left|-k^{2} \int_{\Omega_{s}} \boldsymbol{\tau}: \boldsymbol{s}+k^{2}\left\langle\boldsymbol{\gamma}_{\boldsymbol{n}}(\boldsymbol{\tau}), \boldsymbol{\varphi}\right\rangle_{\Sigma}\right|}{\|\boldsymbol{\tau}\|_{\mathbf{H}\left(\operatorname{div} ; \Omega_{s}\right)}} \\
& \quad \geq \sup _{\boldsymbol{\xi} \in\left[H^{-1 / 2}(\Sigma)\right]^{3} \backslash\{\mathbf{0}\}} \frac{\left|-k^{2} \int_{\Omega_{s}} \boldsymbol{S}(\boldsymbol{\xi}): \boldsymbol{s}+k^{2}\left\langle\boldsymbol{\gamma}_{\boldsymbol{n}}(\boldsymbol{S}(\boldsymbol{\xi})), \boldsymbol{\varphi}\right\rangle_{\Sigma}\right|}{\|\boldsymbol{S}(\boldsymbol{\xi})\|_{\mathbf{H}\left(\operatorname{div} ; \Omega_{s}\right)}} \\
& \quad \geq \frac{k^{2}}{\|\boldsymbol{S}\|}\|\boldsymbol{\varphi}\|_{\left[H^{1 / 2}(\Sigma)\right]^{3}}-k^{2}\|\boldsymbol{s}\|_{\left[L^{2}\left(\Omega_{s}\right)\right]^{3 \times 3} .} .
\end{aligned}
$$

We now let $\boldsymbol{T}:\left[L^{2}\left(\Omega_{s}\right)\right]_{\text {asym }}^{3 \times 3} \rightarrow \mathbf{H}\left(\operatorname{div} ; \Omega_{s}\right)$ be the bounded linear operator defined by

$$
\boldsymbol{T}(\boldsymbol{s}):=\boldsymbol{\varepsilon}(\mathbf{w})-\{\boldsymbol{\varepsilon}(\mathbf{z})+\boldsymbol{s}\} \quad \forall \boldsymbol{s} \in\left[L^{2}\left(\Omega_{s}\right)\right]_{\mathrm{asym}}^{3 \times 3},
$$

where $\mathbf{z} \in\left[H_{0}^{1}\left(\Omega_{s}\right)\right]^{3}$ and $\mathbf{w} \in\left[H^{1}\left(\Omega_{s}\right)\right]^{3}$ are the unique solutions of the boundary value problems

$$
-\operatorname{div} \varepsilon(\mathbf{z})=\operatorname{div} s \quad \text { in } \Omega_{s}, \quad \mathbf{z}=\mathbf{0} \quad \text { on } \Sigma
$$

and

$$
-\operatorname{div} \varepsilon(\mathbf{w})+\mathbf{w}=\mathbf{0} \quad \text { in } \Omega_{s}, \quad \gamma_{\boldsymbol{n}}(\varepsilon(\mathbf{w}))=\gamma_{\boldsymbol{n}}(\varepsilon(\mathbf{z})+s) \quad \text { on } \Sigma .
$$

It follows that $\gamma_{\boldsymbol{n}}(\boldsymbol{T}(\boldsymbol{s}))=0$ on $\Sigma, \frac{1}{2}\left(\boldsymbol{T}(\boldsymbol{s})-\boldsymbol{T}(\boldsymbol{s})^{\mathrm{t}}\right)=-\boldsymbol{s}$ in $\Omega_{s}$, and hence

$$
\begin{gathered}
\sup _{\boldsymbol{\tau} \in \mathbf{H}\left(\mathbf{d i v} ; \Omega_{s}\right) \backslash\{\mathbf{0}\}} \frac{\left|-k^{2} \int_{\Omega_{s}} \boldsymbol{\tau}: \boldsymbol{s}+k^{2}\left\langle\boldsymbol{\gamma}_{\boldsymbol{n}}(\boldsymbol{\tau}), \boldsymbol{\varphi}\right\rangle_{\Sigma}\right|}{\|\boldsymbol{\tau}\|_{\mathbf{H}\left(\mathbf{d i v} ; \Omega_{s}\right)}} \\
\geq \frac{\left|-k^{2} \int_{\Omega_{s}} \boldsymbol{T}(\boldsymbol{s}): \boldsymbol{s}+k^{2}\left\langle\boldsymbol{\gamma}_{\boldsymbol{n}}(\boldsymbol{T}(\boldsymbol{s})), \boldsymbol{\varphi}\right\rangle_{\Sigma}\right|}{\|\boldsymbol{T}(\boldsymbol{s})\|_{\mathbf{H}\left(\operatorname{div} ; \Omega_{s}\right)}} \\
=\frac{k^{2}\|\boldsymbol{s}\|_{\left[L^{2}\left(\Omega_{s}\right)\right]^{3 \times 3}}^{2} \geq \frac{k^{2}}{\|\boldsymbol{T}\|}\|\boldsymbol{s}\|_{\left[L^{2}\left(\Omega_{s}\right)\right]^{3 \times 3} .}}{\| \boldsymbol{s}) \|_{\mathbf{H}\left(\operatorname{div} ; \Omega_{s}\right)}}
\end{gathered}
$$

Copyright $@$ by SIAM. Unauthorized reproduction of this article is prohibited. 
Finally, it is not difficult to see that the estimates (5.28), (5.29), and (5.30) imply (5.27).

We now aim to show that $\mathbf{A}_{0}$ is bijective on the kernel of $\mathbf{B}_{0}$. To this end, we first observe from (5.16), recalling the definition of the coefficient $a$ (cf. (3.4)), that for each $\boldsymbol{W} \in \mathbf{H}_{\Gamma}\left(\mathbf{c u r l} ; \Omega_{m}\right)$ there holds

$$
\operatorname{Re}\left\{\mathbf{a}_{m}^{+}(\boldsymbol{W}, \overline{\boldsymbol{W}})\right\}=\int_{\Omega_{m}}\left\{\frac{\epsilon_{0} \epsilon}{\left.\epsilon^{2}+(\sigma / \omega)^{2}\right)}|\operatorname{curl}(\boldsymbol{W})|^{2}+k^{2} b|\boldsymbol{W}|^{2}\right\},
$$

which, according to the definition of $b$ (cf. (3.4)) and the assumptions (3.1), yields

$$
\operatorname{Re}\left\{\mathbf{a}_{m}^{+}(\boldsymbol{W}, \overline{\boldsymbol{W}})\right\} \geq c_{1}\|\boldsymbol{W}\|_{\mathbf{H}\left(\operatorname{curl} ; \Omega_{m}\right)}^{2} \quad \forall \boldsymbol{W} \in \mathbf{H}_{\Gamma}\left(\operatorname{curl} ; \Omega_{m}\right),
$$

where

$$
c_{1}:=\min \left\{\frac{\epsilon_{0}^{2}}{\bar{\epsilon}^{2}+(\bar{\sigma} / \omega)^{2}}, k^{2}\right\} .
$$

Also, we notice here for later use that

$$
\operatorname{Im}\left\{\mathbf{a}_{m}^{+}(\boldsymbol{W}, \overline{\boldsymbol{W}})\right\}=-\int_{\Omega_{m}} \frac{\epsilon_{0}(\sigma / \omega)}{\left.\epsilon^{2}+(\sigma / \omega)^{2}\right)}|\operatorname{curl}(\boldsymbol{W})|^{2}-k\left\|\boldsymbol{\pi}_{\mathbf{t}}(\boldsymbol{W})\right\|_{\left[L^{2}(\Gamma)\right]^{3}}^{2}
$$

Lemma 5.5. Let $\mathbb{V}$ be the kernel of $\mathbf{B}_{0}$; that is,

$$
\mathbb{V}:=\left\{(\boldsymbol{W}, \boldsymbol{\tau}) \in \mathbb{X}: \quad \mathbf{B}_{0}((\boldsymbol{W}, \boldsymbol{\tau}),(\boldsymbol{\varphi}, \boldsymbol{s}))=0 \forall(\boldsymbol{\varphi}, \boldsymbol{s}) \in \mathbb{M}\right\}
$$

Then, there exists $\alpha>0$ such that

$$
\sup _{(\boldsymbol{W}, \boldsymbol{\tau}) \in \mathbb{V} \backslash\{\mathbf{0}\}} \frac{\left|\mathbf{A}_{0}((\boldsymbol{H}, \boldsymbol{\sigma}),(\boldsymbol{W}, \boldsymbol{\tau}))\right|}{\|(\boldsymbol{W}, \boldsymbol{\tau})\|_{\mathbb{X}}} \geq \alpha\|(\boldsymbol{H}, \boldsymbol{\sigma})\|_{\mathbb{X}} \quad \forall(\boldsymbol{H}, \boldsymbol{\sigma}) \in \mathbb{V} .
$$

In addition, there holds

$$
\sup _{(\boldsymbol{H}, \boldsymbol{\sigma}) \in \mathbb{V}}\left|\mathbf{A}_{0}((\boldsymbol{H}, \boldsymbol{\sigma}),(\boldsymbol{W}, \boldsymbol{\tau}))\right|>0 \quad \forall(\boldsymbol{W}, \boldsymbol{\tau}) \in \mathbb{V} \backslash\{\boldsymbol{0}\} .
$$

Proof. From the definition of $\mathbf{B}_{0}$ (cf. (5.24)) we deduce that $\mathbb{V}=\mathbf{H}_{\Gamma}\left(\mathbf{c u r l} ; \Omega_{m}\right) \times$ $V$, where

$$
V:=\left\{\boldsymbol{\tau} \in \mathbf{H}\left(\operatorname{div} ; \Omega_{s}\right): \quad \boldsymbol{\tau}=\boldsymbol{\tau}^{\mathrm{t}} \text { in } \Omega_{s} \text { and } \gamma_{\boldsymbol{n}}(\boldsymbol{\tau})=\mathbf{0} \text { on } \Sigma\right\} .
$$

Now, given a parameter $\eta>0$ to be chosen later, we let $\Xi: \mathbb{X} \rightarrow \mathbb{X}$ be the linear operator defined by$$
\Xi(\boldsymbol{W}, \boldsymbol{\tau}):=\Xi_{1}(\boldsymbol{W}, \boldsymbol{\tau})+\Xi_{2}(\boldsymbol{W}, \boldsymbol{\tau}) \quad \forall(\boldsymbol{W}, \boldsymbol{\tau}) \in \mathbb{X}:=\mathbf{H}_{\Gamma}\left(\operatorname{curl} ; \Omega_{m}\right) \times \mathbf{H}\left(\operatorname{div} ; \Omega_{s}\right),
$$

where

$(5.38) \Xi_{1}(\boldsymbol{W}, \boldsymbol{\tau}):=\left(\left(2 \mathcal{P}_{m}-\mathcal{I}\right)(\boldsymbol{W}),\left(2 \mathcal{P}_{s}-\mathcal{I}\right)(\boldsymbol{\tau})\right) \quad$ and $\quad \Xi_{2}(\boldsymbol{W}, \boldsymbol{\tau}):=\imath \eta(\boldsymbol{W}, \mathbf{0})$. 
It follows from the boundedness of $\mathcal{P}_{m}$ and $\mathcal{P}_{s}$ (cf. (5.1) and (5.12)) that $\Xi$ is bounded. In addition, since $\mathcal{P}_{s}(\boldsymbol{\tau})$ is symmetric and $\gamma_{\boldsymbol{n}}\left(\mathcal{P}_{s}(\boldsymbol{\tau})\right)=\mathbf{0}$ on $\Sigma$ (see details in section 5.2), we deduce that $\left(2 \mathcal{P}_{s}-\mathcal{I}\right)(\boldsymbol{\tau}) \in V$ for each $\boldsymbol{\tau} \in V$, and hence $\Xi(\boldsymbol{W}, \boldsymbol{\tau}) \in \mathbb{V}$ for each $(\boldsymbol{W}, \boldsymbol{\tau}) \in \mathbb{V}$. Thus, we have in particular that

$$
\sup _{(\boldsymbol{W}, \boldsymbol{\tau}) \in \mathbb{V} \backslash\{\mathbf{0}\}} \frac{\left|\mathbf{A}_{0}((\boldsymbol{H}, \boldsymbol{\sigma}),(\boldsymbol{W}, \boldsymbol{\tau}))\right|}{\|(\boldsymbol{W}, \boldsymbol{\tau})\|_{\mathbb{X}}} \geq \frac{\left|\mathbf{A}_{0}((\boldsymbol{H}, \boldsymbol{\sigma}), \Xi(\overline{\boldsymbol{H}}, \overline{\boldsymbol{\sigma}}))\right|}{\|\Xi(\overline{\boldsymbol{H}}, \overline{\boldsymbol{\sigma}})\|_{\mathbb{X}}}
$$

for all $(\boldsymbol{H}, \boldsymbol{\sigma}) \in \mathbb{V} \backslash\{\mathbf{0}\}$, where

$$
\mathbf{A}_{0}((\boldsymbol{H}, \boldsymbol{\sigma}), \Xi(\overline{\boldsymbol{H}}, \overline{\boldsymbol{\sigma}}))=\mathbf{A}_{0}\left((\boldsymbol{H}, \boldsymbol{\sigma}), \Xi_{1}(\overline{\boldsymbol{H}}, \overline{\boldsymbol{\sigma}})\right)+\mathbf{A}_{0}\left((\boldsymbol{H}, \boldsymbol{\sigma}), \Xi_{2}(\overline{\boldsymbol{H}}, \overline{\boldsymbol{\sigma}})\right) .
$$

Since $\mathcal{P}_{m}^{2}=\mathcal{P}_{m}$ and $\mathcal{P}_{s}^{2}=\mathcal{P}_{s}$, we observe that

$$
\mathcal{P}_{m}\left(2 \mathcal{P}_{m}-\mathcal{I}\right)=\mathcal{P}_{m}, \quad\left(\mathcal{I}-\mathcal{P}_{m}\right)\left(2 \mathcal{P}_{m}-\mathcal{I}\right)=-\left(\mathcal{I}-\mathcal{P}_{m}\right)
$$

and analogously for $\mathcal{P}_{s}$, whence we obtain from (5.21) that

$$
\begin{aligned}
& \mathbf{A}_{0}\left((\boldsymbol{H}, \boldsymbol{\sigma}), \Xi_{1}(\overline{\boldsymbol{H}}, \overline{\boldsymbol{\sigma}})\right)=\mathbf{a}_{m}^{+}\left(\mathcal{P}_{m}(\boldsymbol{H}), \mathcal{P}_{m}(\overline{\boldsymbol{H}})\right)+\mathbf{a}_{m}^{+}\left(\left(\mathcal{I}-\mathcal{P}_{m}\right)(\boldsymbol{H}),\left(\mathcal{I}-\mathcal{P}_{m}\right)(\overline{\boldsymbol{H}})\right) \\
& (5.41) \quad+2 \imath k\left\|\boldsymbol{\pi}_{\mathbf{t}}\left(\left(\mathcal{I}-\mathcal{P}_{m}\right)(\boldsymbol{H})\right)\right\|_{\left[L^{2}(\Gamma)\right]^{3}}^{2}+\mathbf{a}_{s}^{+}\left(\mathcal{P}_{s}(\boldsymbol{\sigma}), \mathcal{P}_{s}(\overline{\boldsymbol{\sigma}})\right) \\
& \quad+\mathbf{a}_{s}^{+}\left(\left(\mathcal{I}-\mathcal{P}_{s}\right)(\boldsymbol{\sigma}),\left(\mathcal{I}-\mathcal{P}_{s}\right)(\overline{\boldsymbol{\sigma}})\right)
\end{aligned}
$$

Applying (5.32) to the first two terms on the right-hand side of (5.41), we deduce that

$$
\begin{aligned}
& \operatorname{Re}\left\{\mathbf{a}_{m}^{+}\left(\mathcal{P}_{m}(\boldsymbol{H}), \mathcal{P}_{m}(\overline{\boldsymbol{H}})\right)+\mathbf{a}_{m}^{+}\left(\left(\mathcal{I}-\mathcal{P}_{m}\right)(\boldsymbol{H}),\left(\mathcal{I}-\mathcal{P}_{m}\right)(\overline{\boldsymbol{H}})\right)\right\} \\
& \geq c_{1}\left\{\left\|\mathcal{P}_{m}(\boldsymbol{H})\right\|_{\mathbf{H}\left(\mathbf{c u r l} ; \Omega_{m}\right)}^{2}+\left\|\left(\mathcal{I}-\mathcal{P}_{m}\right)(\boldsymbol{H})\right\|_{\mathbf{H}\left(\mathbf{c u r l} ; \Omega_{m}\right)}^{2}\right\}
\end{aligned}
$$

for all $\boldsymbol{H} \in \mathbf{H}_{\Gamma}\left(\mathbf{c u r l} ; \Omega_{m}\right)$. Next, employing the same arguments as in the $2 \mathrm{D}$ case (see section 4.2 of [12], particularly Lemmas 4.6 and 4.7), one can show that there exists $c_{2}>0$ such that

$\operatorname{Re}\left\{\mathbf{a}_{s}^{+}\left(\mathcal{P}_{s}(\boldsymbol{\sigma}), \mathcal{P}_{s}(\overline{\boldsymbol{\sigma}})\right)+\mathbf{a}_{s}^{+}\left(\left(\mathcal{I}-\mathcal{P}_{s}\right)(\boldsymbol{\sigma}),\left(\mathcal{I}-\mathcal{P}_{s}\right)(\overline{\boldsymbol{\sigma}})\right)\right\} \geq c_{2}\|\boldsymbol{\sigma}\|_{\mathbf{H}\left(\mathbf{d i v} ; \Omega_{s}\right)}^{2} \quad \forall \boldsymbol{\sigma} \in V$.

In this way, thanks to $(5.41),(5.42)$, and the above inequality, we deduce that

$$
\begin{aligned}
& \operatorname{Re}\left\{\mathbf{A}_{0}\left((\boldsymbol{H}, \boldsymbol{\sigma}), \Xi_{1}(\overline{\boldsymbol{H}}, \overline{\boldsymbol{\sigma}})\right)\right\} \geq\left\{c_{1}\left\|\mathcal{P}_{m}(\boldsymbol{H})\right\|_{\mathbf{H}\left(\mathbf{c u r l} ; \Omega_{m}\right)}^{2}\right. \\
& \left.\quad+c_{1}\left\|\left(\mathcal{I}-\mathcal{P}_{m}\right)(\boldsymbol{H})\right\|_{\mathbf{H}\left(\mathbf{c u r l} ; \Omega_{m}\right)}^{2}+c_{2}\|\boldsymbol{\sigma}\|_{\mathbf{H}\left(\mathbf{d i v} ; \Omega_{s}\right)}^{2}\right\} \quad \forall(\boldsymbol{H}, \boldsymbol{\sigma}) \in \mathbb{V} .
\end{aligned}
$$

On the other hand, it is clear also from (5.21) that

$$
\begin{aligned}
\mathbf{A}_{0} & \left((\boldsymbol{H}, \boldsymbol{\sigma}), \Xi_{2}(\overline{\boldsymbol{H}}, \overline{\boldsymbol{\sigma}})\right) \\
= & \imath \eta\left\{\mathbf{a}_{m}^{+}\left(\mathcal{P}_{m}(\boldsymbol{H}), \mathcal{P}_{m}(\overline{\boldsymbol{H}})\right)-\mathbf{a}_{m}^{+}\left(\left(\mathcal{I}-\mathcal{P}_{m}\right)(\boldsymbol{H}),\left(\mathcal{I}-\mathcal{P}_{m}\right)(\overline{\boldsymbol{H}})\right)\right\} \\
& +2 k \eta\left\|\boldsymbol{\pi}_{\mathbf{t}}\left(\left(\mathcal{I}-\mathcal{P}_{m}\right)(\boldsymbol{H})\right)\right\|_{\left[L^{2}(\Gamma)\right]^{3}}^{2} .
\end{aligned}
$$

Copyright (c) by SIAM. Unauthorized reproduction of this article is prohibited. 
Then, using that $\operatorname{Re}(\imath z)=-\operatorname{Im}(z)$ and (5.33), we obtain that

$$
\begin{aligned}
& \operatorname{Re}\left\{\mathbf{A}_{0}\left((\boldsymbol{H}, \boldsymbol{\sigma}), \Xi_{2}(\overline{\boldsymbol{H}}, \overline{\boldsymbol{\sigma}})\right)\right\}=\eta \int_{\Omega_{m}} \frac{\epsilon_{0}(\sigma / \omega)}{\left.\epsilon^{2}+(\sigma / \omega)^{2}\right)}\left|\operatorname{curl}\left(\mathcal{P}_{m}(\boldsymbol{H})\right)\right|^{2} \\
& \quad+k \eta\left\|\boldsymbol{\pi}_{\mathbf{t}}\left(\mathcal{P}_{m}(\boldsymbol{H})\right)\right\|_{\left[L^{2}(\Gamma)\right]^{3}}^{2}-\eta \int_{\Omega_{m}} \frac{\epsilon_{0}(\sigma / \omega)}{\left.\epsilon^{2}+(\sigma / \omega)^{2}\right)}\left|\operatorname{curl}\left(\left(\mathcal{I}-\mathcal{P}_{m}\right)(\boldsymbol{H})\right)\right|^{2} \\
& \quad+k \eta\left\|\boldsymbol{\pi}_{\mathbf{t}}\left(\left(\mathcal{I}-\mathcal{P}_{m}\right)(\boldsymbol{H})\right)\right\|_{\left[L^{2}(\Gamma)\right]^{3}}^{2},
\end{aligned}
$$

which, thanks to the assumptions (3.1), and defining $C_{2}:=\bar{\sigma} /\left(\epsilon_{0} \omega\right)$, yields

$$
\begin{aligned}
& \operatorname{Re}\left\{\mathbf{A}_{0}\left((\boldsymbol{H}, \boldsymbol{\sigma}), \Xi_{2}(\overline{\boldsymbol{H}}, \overline{\boldsymbol{\sigma}})\right)\right\} \geq\left\{k \eta\left\|\boldsymbol{\pi}_{\mathbf{t}}\left(\mathcal{P}_{m}(\boldsymbol{H})\right)\right\|_{\left[L^{2}(\Gamma)\right]^{3}}^{2}\right. \\
& \left.\quad+k \eta\left\|\boldsymbol{\pi}_{\mathbf{t}}\left(\left(\mathcal{I}-\mathcal{P}_{m}\right)(\boldsymbol{H})\right)\right\|_{\left[L^{2}(\Gamma)\right]^{3}}^{2}-C_{2} \eta\left\|\left(\mathcal{I}-\mathcal{P}_{m}\right)(\boldsymbol{H})\right\|_{\mathbf{H}\left(\mathbf{c u r l} ; \Omega_{m}\right)}^{2}\right\}
\end{aligned}
$$

for all $(\boldsymbol{H}, \boldsymbol{\sigma}) \in \mathbb{V}$. Consequently, having in mind (5.40), adding (5.44) with (5.46), choosing $\eta \in\left(0, c_{1} / C_{2}\right)$, and then applying the equivalence estimate (5.4), we find $\tilde{\alpha}>0$, depending on $c_{1}, c_{2}, k, \eta$, and $C_{2}$, such that

$$
\operatorname{Re}\left\{\mathbf{A}_{0}((\boldsymbol{H}, \boldsymbol{\sigma}), \Xi(\overline{\boldsymbol{H}}, \overline{\boldsymbol{\sigma}}))\right\} \geq \tilde{\alpha}\|(\boldsymbol{H}, \boldsymbol{\sigma})\|_{\mathbb{X}}^{2} \quad \forall(\boldsymbol{H}, \boldsymbol{\sigma}) \in \mathbb{V},
$$

which, using the boundedness of $\Xi$, yields the existence of $\alpha>0$, depending on $\tilde{\alpha}$ and $\|\Xi\|$, such that

$$
\frac{\left|\mathbf{A}_{0}((\boldsymbol{H}, \boldsymbol{\sigma}), \Xi(\overline{\boldsymbol{H}}, \overline{\boldsymbol{\sigma}}))\right|}{\|\Xi(\overline{\boldsymbol{H}}, \overline{\boldsymbol{\sigma}})\|_{\mathbb{X}}} \geq \alpha\|(\boldsymbol{H}, \boldsymbol{\sigma})\|_{\mathbb{X}} \quad \forall(\boldsymbol{H}, \boldsymbol{\sigma}) \in \mathbb{V} \backslash\{\mathbf{0}\}
$$

The above estimate and (5.39) prove the inf-sup condition (5.34). Finally, the symmetry of $\mathbf{A}_{0}$ and (5.34) provide the inf-sup condition (5.35).

We remark here that the purpose of introducing the parameter $\eta$ in the definition of $\Xi_{2}$ (cf. (5.38)) is twofold. On the one hand, a positive value of $\eta$ makes the boundary term $\left\|\boldsymbol{\pi}_{\mathbf{t}}\left(\left(\mathcal{I}-\mathcal{P}_{m}\right)(\boldsymbol{H})\right)\right\|_{\left[L^{2}(\Gamma)\right]^{3}}^{2}$ appear (see (5.46)), which is needed to define $\left\|\left(\mathcal{I}-\mathcal{P}_{m}\right)(\boldsymbol{H})\right\|_{\mathbf{H}_{\Gamma}\left(\operatorname{curl} ; \Omega_{m}\right)}^{2}$ (cf. (4.1)). It is important to note that $\| \boldsymbol{\pi}_{\mathbf{t}}((\mathcal{I}-$ $\left.\left.\mathcal{P}_{m}\right)(\boldsymbol{H})\right) \|_{\left[L^{2}(\Gamma)\right]^{3}}^{2}$ is not a compact term, and hence it cannot be simply added and subtracted. On the other hand, the addition of (5.44) and (5.46) yields the expression $\left(c_{1}-C_{2} \eta\right)\left\|\left(\mathcal{I}-\mathcal{P}_{m}\right)(\boldsymbol{H})\right\|_{\mathbf{H}\left(\mathbf{c u r l} ; \Omega_{m}\right)}^{2}$, and then a sufficiently small $\eta$ allows us to derive the bound

$$
\left(c_{1}-C_{2} \eta\right)\left\|\left(\mathcal{I}-\mathcal{P}_{m}\right)(\boldsymbol{H})\right\|_{\mathbf{H}\left(\mathbf{c u r l} ; \Omega_{m}\right)}^{2} \geq c\left\|\left(\mathcal{I}-\mathcal{P}_{m}\right)(\boldsymbol{H})\right\|_{\mathbf{H}\left(\mathbf{c u r l} ; \Omega_{m}\right)}^{2},
$$

which provides the domain term defining $\left\|\left(\mathcal{I}-\mathcal{P}_{m}\right)(\boldsymbol{H})\right\|_{\mathbf{H}_{\Gamma}\left(\mathbf{c u r l} ; \Omega_{m}\right)}^{2}(\mathrm{cf}$. (4.1)). Finally, we notice that using the present definition of $\Xi$ (cf. (5.37)) is equivalent to employing

$$
\Xi(\mathbf{W}, \boldsymbol{\tau}):=(1-\eta \imath)\left(\left(2 \mathcal{P}_{m}-\mathcal{I}\right)(\mathbf{W}),\left(2 \mathcal{P}_{s}-\mathcal{I}\right)(\boldsymbol{\tau})\right) .
$$

As a consequence of Lemmas 5.4 and 5.5 and the well-known Babuška-Brezzi theory, we deduce that the matrix operator $\left(\begin{array}{cc}\mathbb{A}_{0} & \mathbb{B}_{0}^{*} \\ \mathbb{B}_{0} & 0\end{array}\right): \mathbb{X} \times \mathbb{M} \rightarrow \mathbb{X} \times \mathbb{M}$ becomes an isomorphism.

Lemma 5.6. The operators $\mathbb{K}_{0}: \mathbb{X} \rightarrow \mathbb{X}$ and $\mathbb{K}_{1}: \mathbb{X} \rightarrow \mathbb{M}$ are compact. 
Proof. We first observe from (5.22) that the bilinear form $\mathbf{K}_{0}$ can be decomposed as

$$
\mathbf{K}_{0}((\boldsymbol{H}, \boldsymbol{\sigma}),(\boldsymbol{W}, \boldsymbol{\tau}))=\mathbf{k}_{m}(\boldsymbol{H}, \boldsymbol{W})+\mathbf{k}_{s}(\boldsymbol{\sigma}, \boldsymbol{\tau})
$$

where

$$
\begin{aligned}
\mathbf{k}_{m}(\boldsymbol{H}, \boldsymbol{W}) & :=2 \mathbf{a}_{m}^{+}\left(\left(\mathcal{I}-\mathcal{P}_{m}\right)(\boldsymbol{H}),\left(\mathcal{I}-\mathcal{P}_{m}\right)(\boldsymbol{W})\right)+\mathbf{a}_{m}^{+}\left(\mathcal{P}_{m}(\boldsymbol{H}),\left(\mathcal{I}-\mathcal{P}_{m}\right)(\boldsymbol{W})\right) \\
& +2 \imath k \int_{\Gamma} \boldsymbol{\pi}_{\mathbf{t}}\left(\left(\mathcal{I}-\mathcal{P}_{m}\right)(\boldsymbol{H})\right) \cdot \boldsymbol{\pi}_{\mathbf{t}}\left(\left(\mathcal{I}-\mathcal{P}_{m}\right)(\boldsymbol{W})\right) \\
& +\mathbf{a}_{m}^{+}\left(\left(\mathcal{I}-\mathcal{P}_{m}\right)(\boldsymbol{H}), \mathcal{P}_{m}(\boldsymbol{W})\right)-2 k^{2} \int_{\Omega_{m}} b \boldsymbol{H} \cdot \boldsymbol{W}
\end{aligned}
$$

and

$$
\begin{aligned}
\mathbf{k}_{s}(\boldsymbol{\sigma}, \boldsymbol{\tau}) & :=2 \mathbf{a}_{s}^{+}\left(\left(\mathcal{I}-\mathcal{P}_{s}\right)(\boldsymbol{\sigma}),\left(\mathcal{I}-\mathcal{P}_{s}\right)(\boldsymbol{\tau})\right)+\mathbf{a}_{s}^{+}\left(\mathcal{P}_{s}(\boldsymbol{\sigma}),\left(\mathcal{I}-\mathcal{P}_{s}\right)(\boldsymbol{\tau})\right) \\
+ & \mathbf{a}_{s}^{+}\left(\left(\mathcal{I}-\mathcal{P}_{s}\right)(\boldsymbol{\sigma}), \mathcal{P}_{s}(\boldsymbol{\tau})\right)-2 k^{2} \int_{\Omega_{s}} \mathcal{C}^{-1} \boldsymbol{\sigma}: \boldsymbol{\tau}
\end{aligned}
$$

Then, according to the definition of $\mathbf{a}_{m}^{+}$(cf. (5.16)), and using that

$$
\operatorname{curl}\left(\left(\mathcal{I}-\mathcal{P}_{m}\right)(\boldsymbol{W})\right)=\mathbf{0} \quad \forall \boldsymbol{W} \in \mathbf{H}_{\Gamma}\left(\operatorname{curl} ; \Omega_{m}\right),
$$

we find that

$$
\begin{aligned}
& \mathbf{k}_{m}(\boldsymbol{H}, \boldsymbol{W})=2 k^{2} \int_{\Omega_{m}} b\left(\mathcal{I}-\mathcal{P}_{m}\right)(\boldsymbol{H}) \cdot\left(\mathcal{I}-\mathcal{P}_{m}\right)(\boldsymbol{W}) \\
& \quad+k^{2} \int_{\Omega_{m}} b \mathcal{P}_{m}(\boldsymbol{H}) \cdot\left(\mathcal{I}-\mathcal{P}_{m}\right)(\boldsymbol{W})-\imath k \int_{\Gamma} \boldsymbol{\pi}_{\mathbf{t}}\left(\mathcal{P}_{m}(\boldsymbol{H})\right) \cdot \boldsymbol{\pi}_{\mathbf{t}}\left(\left(\mathcal{I}-\mathcal{P}_{m}\right)(\boldsymbol{W})\right) \\
& \quad+k^{2} \int_{\Omega_{m}} b\left(\mathcal{I}-\mathcal{P}_{m}\right)(\boldsymbol{H}) \cdot \mathcal{P}_{m}(\boldsymbol{W})-\imath k \int_{\Gamma} \boldsymbol{\pi}_{\mathbf{t}}\left(\left(\mathcal{I}-\mathcal{P}_{m}\right)(\boldsymbol{H})\right) \cdot \boldsymbol{\pi}_{\mathbf{t}}\left(\mathcal{P}_{m}(\boldsymbol{W})\right) \\
& \quad-2 k^{2} \int_{\Omega_{m}} b \boldsymbol{H} \cdot \boldsymbol{W},
\end{aligned}
$$

which, after simplifying the terms involving integration on $\Omega_{m}$, yields

$$
\begin{aligned}
& \mathbf{k}_{m}(\boldsymbol{H}, \boldsymbol{W})=-k^{2} \int_{\Omega_{m}} b\left\{2 \mathcal{P}_{m}(\boldsymbol{H}) \cdot \mathcal{P}_{m}(\boldsymbol{W})+\mathcal{P}_{m}(\boldsymbol{H}) \cdot\left(\mathcal{I}-\mathcal{P}_{m}\right)(\boldsymbol{W})\right. \\
& \left.+\left(\mathcal{I}-\mathcal{P}_{m}\right)(\boldsymbol{H}) \cdot \mathcal{P}_{m}(\boldsymbol{W})\right\}-\imath k \int_{\Gamma} \boldsymbol{\pi}_{\mathbf{t}}\left(\mathcal{P}_{m}(\boldsymbol{H})\right) \cdot \boldsymbol{\pi}_{\mathbf{t}}\left(\left(\mathcal{I}-\mathcal{P}_{m}\right)(\boldsymbol{W})\right) \\
& \quad-\imath k \int_{\Gamma} \boldsymbol{\pi}_{\mathbf{t}}\left(\left(\mathcal{I}-\mathcal{P}_{m}\right)(\boldsymbol{H})\right) \cdot \boldsymbol{\pi}_{\mathbf{t}}\left(\mathcal{P}_{m}(\boldsymbol{W})\right) .
\end{aligned}
$$

The compactness of $\mathcal{P}_{m}: \mathbf{H}_{\Gamma}\left(\mathbf{c u r l} ; \Omega_{m}\right) \rightarrow\left[L^{2}\left(\Omega_{m}\right)\right]^{3}$ guarantees that the operator associated with the integrals on $\Omega_{m}$ is compact, whereas the compact imbedding $\boldsymbol{\pi}_{\mathbf{t}}\left(\left[H^{1}\left(\Omega_{m}\right)\right]^{3}\right):=\mathbf{H}_{\|}^{1 / 2}(\Gamma) \hookrightarrow\left[L^{2}(\Gamma)\right]^{3}$ (cf. [17, Lemma 3.2]) implies that $\boldsymbol{\pi}_{\mathbf{t}} \circ \mathcal{P}_{m}:$ $\mathbf{H}_{\Gamma}\left(\mathbf{c u r l} ; \Omega_{m}\right) \rightarrow\left[L^{2}(\Gamma)\right]^{3}$, and hence the operator associated with the integrals on $\Gamma$, are both compact.

Copyright $@$ by SIAM. Unauthorized reproduction of this article is prohibited. 
On the other hand, since $\operatorname{div}\left(\mathcal{I}-\mathcal{P}_{s}\right)(\boldsymbol{\tau})=\mathbf{M}(\operatorname{div}(\boldsymbol{\tau})), \mathcal{P}_{s}(\boldsymbol{\tau})$ is symmetric, $\gamma_{\boldsymbol{n}}\left(\mathcal{P}_{s}(\boldsymbol{\tau})\right)=0$ on $\Sigma \forall \boldsymbol{\tau} \in \mathbf{H}\left(\mathbf{d i v} ; \Omega_{s}\right)$, and $\nabla \mathbf{v} \in\left[L^{2}\left(\Omega_{s}\right)\right]_{\text {asym }}^{3 \times 3} \forall \mathbf{v} \in \mathbb{R M}\left(\Omega_{s}\right)$ (see section 5.2 for details), we deduce that

$$
\begin{gathered}
\int_{\Omega_{s}} \operatorname{div}\left(\mathcal{I}-\mathcal{P}_{s}\right)(\sigma) \cdot \operatorname{div}\left(\mathcal{P}_{s}(\tau)\right)=\int_{\Omega_{s}} \mathbf{M}(\operatorname{div}(\sigma)) \cdot \operatorname{div}\left(\mathcal{P}_{s}(\tau)\right) \\
=-\int_{\Omega_{s}} \nabla \mathbf{M}(\operatorname{div}(\sigma)): \mathcal{P}_{s}(\tau)+\left\langle\gamma_{\boldsymbol{n}}\left(\mathcal{P}_{s}(\boldsymbol{\tau})\right), \mathbf{M}(\operatorname{div}(\boldsymbol{\sigma}))\right\rangle_{\Sigma}=0
\end{gathered}
$$

for all $\boldsymbol{\sigma}, \boldsymbol{\tau} \in \mathbf{H}\left(\operatorname{div} ; \Omega_{s}\right)$. It follows, recalling the definition of $\mathbf{a}_{s}^{+}$(cf. (5.17)), that

$$
\begin{aligned}
& \mathbf{k}_{s}(\boldsymbol{\sigma}, \boldsymbol{\tau})=-2 k^{2} \int_{\Omega_{s}} \mathcal{C}^{-1} \mathcal{P}_{s}(\boldsymbol{\sigma}): \mathcal{P}_{s}(\boldsymbol{\tau})-k^{2} \int_{\Omega_{s}} \mathcal{C}^{-1} \mathcal{P}_{s}(\boldsymbol{\sigma}):\left(\mathcal{I}-\mathcal{P}_{s}\right)(\boldsymbol{\tau}) \\
& -k^{2} \int_{\Omega_{s}} \mathcal{C}^{-1}\left(\mathcal{I}-\mathcal{P}_{s}\right)(\boldsymbol{\sigma}): \mathcal{P}_{s}(\boldsymbol{\tau})+\frac{2 k^{2}}{\kappa_{s}^{2}} \int_{\Omega_{s}} \operatorname{div}\left(\mathcal{I}-\mathcal{P}_{s}\right)(\boldsymbol{\sigma}) \cdot \operatorname{div}\left(\mathcal{I}-\mathcal{P}_{s}\right)(\boldsymbol{\tau}),
\end{aligned}
$$

which, thanks to Lemma 5.3, shows that the operator induced by $\mathbf{k}_{s}$ is compact.

Finally, the definition of $\mathbf{K}_{1}$ (cf. (5.25)) and the compact imbedding $\mathbf{H}_{\|}^{1 / 2}(\Sigma) \hookrightarrow$ $\left[L^{2}(\Sigma)\right]^{3}$ (cf. [17, Lemma 3.2]) yield the compactness of $\mathbb{K}_{1}$.

We are now ready to establish the main result of this section.

TheOREm 5.1. Assume that the homogeneous problem associated with (4.10) has only the trivial solution. Then, given $\boldsymbol{H}^{i}, \boldsymbol{E}^{i} \in \mathbf{H}\left(\mathbf{c u r l} ; \Omega_{m}\right)$, there exists a unique solution $((\boldsymbol{H}, \boldsymbol{\sigma}),(\boldsymbol{\psi}, \boldsymbol{r})) \in \mathbb{X} \times \mathbb{M}$ to (4.10). In addition, there exists $C>0$ such that

$$
\|((\boldsymbol{H}, \boldsymbol{\sigma}),(\boldsymbol{\psi}, \boldsymbol{r}))\|_{\mathbb{X} \times \mathbb{M}} \leq C\|(\mathbf{L}, \mathbf{0})\|_{\mathbb{X}^{\prime} \times \mathbb{M}^{\prime}} .
$$

Proof. It suffices to observe, in virtue of Lemmas 5.4, 5.5, and 5.6, that the left-hand side of (5.26) constitutes a Fredholm operator of index zero, and hence the well-posedness of (4.10) follows from uniqueness.

We end this section with a uniqueness result for (4.10). Indeed, let us first notice that there may exist singular frequencies $\omega$ for which the homogeneous problem

$$
\begin{gathered}
\boldsymbol{\sigma}=\mathcal{C} \boldsymbol{\varepsilon}(\boldsymbol{u}) \text { in } \Omega_{s}, \quad \operatorname{div} \boldsymbol{\sigma}+\omega^{2} \rho_{s} \boldsymbol{u}=\mathbf{0} \quad \text { in } \Omega_{s}, \\
\boldsymbol{u} \times \boldsymbol{n}=\mathbf{0} \quad \text { on } \Sigma, \quad \boldsymbol{\sigma} \boldsymbol{n}=\mathbf{0} \quad \text { on } \Sigma,
\end{gathered}
$$

which arises from (3.12) assuming that $\boldsymbol{H}=\boldsymbol{H}^{i}=\boldsymbol{E}^{i}=\mathbf{0}$, admits a nontrivial solution.

At this point we recall that, thanks to our assumptions on $\Omega_{s}$ and $\Gamma, \Omega_{m}$ is a connected and simply connected Lipschitz polyhedra with boundary $\partial \Omega_{m}$ consisting of two disjoint connected components $\Sigma$ and $\Gamma$. Furthermore, in what follows we assume that $\Omega_{m}$ can be decomposed into $J$ connected polyhedra $\Omega_{m}^{j}$ such that $\bar{\Omega}_{m}=\cup_{j=1}^{J} \bar{\Omega}_{m}^{j}$ and $\Omega_{m}^{i} \cap \Omega_{m}^{j}=\emptyset$ if $i \neq j$. Then we have the following result.

THEOREM 5.2. Assume that (5.50) admits only the trivial solution. In addition, suppose that the magnetic permeability $\mu$ is constant on each subdomain $\Omega_{m}^{j}$ and that the restrictions of $\epsilon$ and $\sigma$ to $\Omega_{m}^{j}$ belong to $H^{3}\left(\Omega_{m}^{j}\right)$ for all $j \in\{1, \ldots, J\}$. Then there is at most one solution to (4.10).

Proof. Let $((\boldsymbol{H}, \boldsymbol{\sigma}),(\boldsymbol{\psi}, \boldsymbol{r}))$ be a solution of the homogeneous system corresponding to (4.10), that is, when $\boldsymbol{H}^{i}=\boldsymbol{E}^{i}=\mathbf{0}$. Then, taking $\boldsymbol{W}=\overline{\boldsymbol{H}}$ in (4.2) gives

$$
\begin{aligned}
\int_{\Omega_{m}} & \left\{a^{-1}|\operatorname{curl}(\boldsymbol{H})|^{2}-k^{2} b|\boldsymbol{H}|^{2}\right\}+\left\langle\boldsymbol{\gamma}_{\mathbf{t}}\left(a^{-1} \operatorname{curl}(\boldsymbol{H})\right), \boldsymbol{\pi}_{\mathbf{t}}(\overline{\boldsymbol{H}})\right\rangle_{\mathbf{t}, \Sigma} \\
& -\left\langle\boldsymbol{\gamma}_{\mathbf{t}}(\operatorname{curl}(\boldsymbol{H})), \boldsymbol{\pi}_{\mathbf{t}}(\overline{\boldsymbol{H}})\right\rangle_{\mathbf{t}, \Gamma}=0
\end{aligned}
$$

Copyright $@$ by SIAM. Unauthorized reproduction of this article is prohibited. 
which, employing the boundary condition $\gamma_{\mathbf{t}}(\operatorname{curl}(\boldsymbol{H}))=\imath k \boldsymbol{H}$ on $\Gamma$ (cf. (3.15)) in the last term, leads to

$$
\begin{aligned}
\int_{\Omega_{m}} & \left\{a^{-1}|\operatorname{curl}(\boldsymbol{H})|^{2}-k^{2} b|\boldsymbol{H}|^{2}\right\}+\left\langle\boldsymbol{\gamma}_{\mathbf{t}}\left(a^{-1} \operatorname{curl}(\boldsymbol{H})\right), \boldsymbol{\pi}_{\mathbf{t}}(\overline{\boldsymbol{H}})\right\rangle_{\mathbf{t}, \Sigma} \\
& -\imath k\left\|\boldsymbol{\pi}_{\mathbf{t}}(\boldsymbol{H})\right\|_{\left[L^{2}(\Gamma)\right]^{3}}^{2}=0 .
\end{aligned}
$$

Now, from the transmission conditions (3.13) and (3.14) we have, respectively, that

$$
\boldsymbol{\pi}_{\mathbf{t}}\left(a^{-1} \operatorname{curl}(\boldsymbol{H})\right)=-\imath k \boldsymbol{\pi}_{\mathbf{t}}(\boldsymbol{u}) \quad \text { and } \quad \boldsymbol{\gamma}_{\mathbf{t}}(\overline{\boldsymbol{H}})=-\imath k \boldsymbol{\gamma}_{\boldsymbol{n}}(\overline{\boldsymbol{\sigma}}) \quad \text { on } \Sigma,
$$

and hence, using also (2.4) and the fact that $\gamma_{\boldsymbol{n}}(\boldsymbol{\sigma})$ is tangential on $\Sigma$, we deduce that

$$
\begin{gathered}
\left\langle\boldsymbol{\gamma}_{\mathbf{t}}\left(a^{-1} \operatorname{curl}(\boldsymbol{H})\right), \boldsymbol{\pi}_{\mathbf{t}}(\overline{\boldsymbol{H}})\right\rangle_{\mathbf{t}, \Sigma}=-\left\langle\boldsymbol{\gamma}_{\mathbf{t}}(\overline{\boldsymbol{H}}), \boldsymbol{\pi}_{\mathbf{t}}\left(a^{-1} \operatorname{curl}(\boldsymbol{H})\right)\right\rangle_{\mathbf{t}, \Sigma} \\
=k^{2}\left\langle\overline{\boldsymbol{\sigma}} \boldsymbol{n}, \boldsymbol{\pi}_{\mathbf{t}}(\boldsymbol{u})\right\rangle_{\Sigma}=k^{2}\langle\overline{\boldsymbol{\sigma}} \boldsymbol{n}, \boldsymbol{u}\rangle_{\Sigma}=k^{2}\left\{\int_{\Omega_{s}} \mathcal{C} \varepsilon(\overline{\boldsymbol{u}}): \varepsilon(\boldsymbol{u})-\kappa_{s}^{2}\|\boldsymbol{u}\|_{\left[L^{2}\left(\Omega_{s}\right)\right]^{3}}^{2}\right\},
\end{gathered}
$$

where the last identity arises after multiplying the equilibrium equation $\operatorname{div}(\overline{\boldsymbol{\sigma}})+$ $\kappa_{s}^{2} \overline{\boldsymbol{u}}=\mathbf{0}$ in $\Omega_{s}$ by $\boldsymbol{u}$ and integrating by parts. This shows that the expression $\left\langle\gamma_{\mathbf{t}}\left(a^{-1} \operatorname{curl}(\boldsymbol{H})\right), \boldsymbol{\pi}_{\mathbf{t}}(\overline{\boldsymbol{H}})\right\rangle_{\mathbf{t}, \Sigma}$ is real, and, consequently, the imaginary part of (5.51) reduces to

$$
\int_{\Omega_{m}} \operatorname{Im}\left(a^{-1}\right)|\operatorname{curl}(\boldsymbol{H})|^{2}-k\left\|\boldsymbol{\pi}_{\mathbf{t}}(\boldsymbol{H})\right\|_{\left[L^{2}(\Gamma)\right]^{3}}^{2}=0
$$

which, noting that $\operatorname{Im}\left(a^{-1}\right)<0$, implies that $\boldsymbol{\pi}_{\mathbf{t}}(\boldsymbol{H})=\mathbf{0}$ on $\Gamma$. Thus, applying the unique continuation principle (see, e.g., [21, Theorem 4.12]), which makes use of our hypotheses on $\mu, \epsilon$, and $\sigma$, we deduce that $\boldsymbol{H}=\mathbf{0}$ in $\Omega_{m}$. Finally, the fact that $\omega$ is not a singular frequency for problem (5.50) completes the proof.

6. The discrete problem. In order to introduce a Galerkin approximation of (4.10) we first let $\left\{\mathcal{T}_{h}\right\}_{h>0}$ be a regular family of triangulations of $\bar{\Omega}_{s} \cup \bar{\Omega}_{m}$ by tetrahedrons $K$ of diameter $h_{K}$, and assume that, given $l \in\{s, m\}, \mathcal{T}_{h}\left(\Omega_{l}\right):=$ $\left\{K \in \mathcal{T}_{h}: K \subseteq \bar{\Omega}_{l}\right\}$ is a triangulation of $\bar{\Omega}_{l}$. As usual, the parameter $h$ denotes in each case the mesh size of the corresponding triangulation. Then, we denote by $\mathcal{T}_{h}(\Sigma)$ and $\mathcal{T}_{h}(\Gamma)$ the triangulations induced by $\mathcal{T}_{h}\left(\Omega_{m}\right)$ on $\Sigma$ and $\Gamma$, respectively. Also, for reasons that will become clear below, we introduce an independent triangulation $\mathcal{T}_{\tilde{h}}(\Sigma)$ of the interface $\Sigma$ by triangles $\tilde{T}$ of diameter $\tilde{h}_{T}$ and define $\tilde{h}:=\max \left\{\tilde{h}_{T}: \tilde{T} \in \mathcal{T}_{\tilde{h}}(\Sigma)\right\}$.

In what follows, given an integer $\ell \geq 0$ and a subset $\mathcal{D}$ of $\mathbb{R}^{3}, \mathbb{P}_{\ell}(\mathcal{D})$ denotes the space of complex valued polynomials defined in $\mathcal{D}$ of total degree $\leq \ell$. Also, we introduce the finite element spaces

$$
\begin{aligned}
\mathcal{S}_{h}^{1}\left(\Omega_{s}\right):=\left\{v \in \mathcal{C}^{0}\left(\bar{\Omega}_{s}\right):\right. & \left.\left.v\right|_{K} \in \mathbb{P}_{1}(K) \forall K \in \mathcal{T}_{h}\left(\Omega_{s}\right)\right\}, \\
\mathcal{S}_{h}^{0}\left(\Omega_{s}\right):=\left\{v \in L^{2}\left(\Omega_{s}\right):\right. & \left.\left.v\right|_{K} \in \mathbb{P}_{0}(K) \forall K \in \mathcal{T}_{h}\left(\Omega_{s}\right)\right\}, \\
\mathcal{S}_{h}^{1}(\Sigma):=\left\{\varphi \in \mathcal{C}^{0}(\Sigma):\right. & \left.\left.\varphi\right|_{T} \in \mathbb{P}_{1}(T) \forall T \in \mathcal{T}_{h}(\Sigma)\right\} \\
\mathcal{S}_{\tilde{h}}^{1}(\Sigma):=\left\{\varphi \in \mathcal{C}^{0}(\Sigma):\right. & \left.\left.\varphi\right|_{\tilde{T}} \in \mathbb{P}_{1}(\tilde{T}) \forall \tilde{T} \in \tilde{\mathcal{T}}_{\tilde{h}}(\Sigma)\right\}
\end{aligned}
$$

Copyright (c) by SIAM. Unauthorized reproduction of this article is prohibited. 
and

$$
\mathcal{S}_{h}^{0}(\Sigma):=\left\{\xi \in L^{2}(\Sigma):\left.\quad \xi\right|_{T} \in \mathbb{P}_{0}(T) \forall T \in \mathcal{T}_{h}(\Sigma)\right\} .
$$

In addition, for any $K \in \mathcal{T}_{h}\left(\Omega_{s}\right)$ we let $\mathbb{R T}_{0}(K):=\left[\mathbb{P}_{0}(K)\right]^{3} \oplus \mathbb{P}_{0}(K) \boldsymbol{x}$ be the local Raviart-Thomas space of lowest order (cf. [9]), that is,

$$
\mathbb{R} \mathbb{T}_{0}(K):=\left\{v: K \rightarrow \mathbb{C}^{3}, v(\boldsymbol{x})=\boldsymbol{a}+b \boldsymbol{x} \forall \boldsymbol{x} \in K, \boldsymbol{a} \in \mathbb{C}^{3}, b \in \mathbb{C}\right\},
$$

and denote by $b_{K}$ the usual bubble function on $K \in \mathcal{T}_{h}\left(\Omega_{s}\right)$, that is, $b_{K}=\prod_{i=1}^{4} \lambda_{i}^{K}$, where $\left\{\lambda_{i}^{K}\right\}_{i=1}^{4}$ are the barycentric coordinates of $K$. Then, the finite element subspace corresponding to the unknown $\boldsymbol{\sigma}$ is given by

$$
\mathbf{X}_{h}^{s}:=\mathcal{R T}_{h}\left(\Omega_{s}\right) \oplus \mathcal{B}_{h},
$$

where

$$
\mathcal{R T}_{h}\left(\Omega_{s}\right):=\left\{\boldsymbol{\tau} \in \mathbf{H}\left(\operatorname{div} ; \Omega_{s}\right):\left.\left(\boldsymbol{\tau}_{i}\right)^{\mathrm{t}}\right|_{K} \in \mathbb{R T}_{0}(K) \forall i \in\{1,2,3\}, \forall K \in \mathcal{T}_{h}\left(\Omega_{s}\right)\right\}
$$

and

$$
\mathcal{B}_{h}:=\left\{\boldsymbol{\tau}: \Omega_{s} \rightarrow \mathbb{C}^{3 \times 3}:\left.\quad\left(\boldsymbol{\tau}_{i}\right)^{\mathrm{t}}\right|_{K} \in \operatorname{curl}\left(b_{K}\left[\mathbb{P}_{0}(K)\right]^{3}\right) \forall K \in \mathcal{T}_{h}\left(\Omega_{s}\right)\right\},
$$

$\boldsymbol{\tau}_{i}$ being the $i$ th row of $\boldsymbol{\tau}$.

On the other hand, for any $K \in \mathcal{T}_{h}\left(\Omega_{m}\right)$ we let

$$
\mathbb{N D}_{1}(K):=\left[\mathbb{P}_{0}(K)\right]^{3} \oplus\left[\mathbb{P}_{0}(K)\right]^{3} \times \boldsymbol{x}
$$

be the local edge space of Nédélec; that is,

$$
\mathbb{N D}_{1}(K):=\left\{v: K \rightarrow \mathbb{C}^{3}, v(\boldsymbol{x})=\boldsymbol{a}+\boldsymbol{b} \times \boldsymbol{x} \forall \boldsymbol{x} \in K, \boldsymbol{a}, \boldsymbol{b} \in \mathbb{C}^{3}\right\} .
$$

Then, the finite element subspace for the unknown $\boldsymbol{H}$ is defined by

$$
\mathbf{X}_{h}^{m}:=\left\{\boldsymbol{W} \in \mathbf{H}\left(\operatorname{curl} ; \Omega_{m}\right):\left.\quad \boldsymbol{W}\right|_{K} \in \mathbb{N D}_{1}(K) \forall K \in \mathcal{T}_{h}\left(\Omega_{m}\right)\right\} .
$$

For the remaining unknowns $\boldsymbol{r}$ and $\boldsymbol{\psi}$ we introduce, respectively, the subspaces

$$
\mathbf{M}_{h}:=\left\{\left(\begin{array}{ccc}
0 & s_{1} & s_{2} \\
-s_{1} & 0 & s_{3} \\
-s_{2} & -s_{3} & 0
\end{array}\right): s_{i} \in \mathcal{S}_{h}^{1}\left(\Omega_{s}\right) \forall i \in\{1,2,3\}\right\} \quad \text { and } \quad \mathbf{M}_{\tilde{h}}:=\left[\mathcal{S}_{\tilde{h}}^{1}(\Sigma)\right]^{3} .
$$

We recall here that $\mathbf{X}_{h}^{s} \times\left[\mathcal{S}_{h}^{0}\left(\Omega_{s}\right)\right]^{3} \times \mathbf{M}_{h}$ constitutes the well-known PEERS method introduced in [3] for a mixed finite element approximation of the linear elasticity problem in the plane, which was generalized to the 3D case in [19].

Finally, we let

$$
\mathbb{X}_{h}:=\mathbf{X}_{h}^{m} \times \mathbf{X}_{h}^{s}, \quad \mathbb{M}_{\tilde{h}, h}:=\mathbf{M}_{\tilde{h}} \times \mathbf{M}_{h}
$$

and define the mixed finite element scheme associated with our coupled problem (4.10) as follows: Find $\left(\left(\boldsymbol{H}_{h}, \boldsymbol{\sigma}_{h}\right),\left(\boldsymbol{\psi}_{\tilde{h}}, \boldsymbol{r}_{h}\right)\right) \in \mathbb{X}_{h} \times \mathbb{M}_{\tilde{h}, h}$ such that

$$
\begin{aligned}
\mathbf{A}\left(\left(\boldsymbol{H}_{h}, \boldsymbol{\sigma}_{h}\right),(\boldsymbol{W}, \boldsymbol{\tau})\right)+\mathbf{B}\left((\boldsymbol{W}, \boldsymbol{\tau}),\left(\boldsymbol{\psi}_{\tilde{h}}, \boldsymbol{r}_{h}\right)\right)=\mathbf{L}((\boldsymbol{W}, \boldsymbol{\tau})) & & \forall(\boldsymbol{W}, \boldsymbol{\tau}) \in \mathbb{X}_{h} \\
\mathbf{B}\left(\left(\boldsymbol{H}_{h}, \boldsymbol{\sigma}_{h}\right),(\boldsymbol{\varphi}, \boldsymbol{s})\right)=0 & & \forall(\boldsymbol{\varphi}, \boldsymbol{s}) \in \mathbb{M}_{\tilde{h}, h}
\end{aligned}
$$

Copyright $\odot$ by SIAM. Unauthorized reproduction of this article is prohibited. 
7. Analysis of the discrete problem. In this section we analyze the discrete problem (6.4). We first provide several technical results in section 7.1. Then, in section 7.2 we apply a classical theorem on projection methods for Fredholm operators of index zero to show that (6.4) is well-posed.

7.1. Preliminaries. We let $\Pi_{h}^{s}:\left[H^{1}\left(\Omega_{s}\right)\right]^{3 \times 3} \rightarrow \mathcal{R T}_{h}\left(\Omega_{s}\right)$ be the usual Raviart-Thomas equilibrium interpolation operator (see [9]). We recall that $\Pi_{h}^{s}$ is characterized by the identities

$$
\int_{E} \Pi_{h}^{s}(\boldsymbol{\tau}) \boldsymbol{n}=\int_{E} \boldsymbol{\tau} \boldsymbol{n} \quad \forall \text { edges } E \text { of } \mathcal{T}_{h}\left(\Omega_{s}\right),
$$

which yield the commuting diagram property

$$
\operatorname{div}\left(\Pi_{h}^{s}(\boldsymbol{\tau})\right)=\mathcal{Q}_{h}^{s}(\operatorname{div}(\boldsymbol{\tau})) \quad \forall \boldsymbol{\tau} \in\left[H^{1}\left(\Omega_{s}\right)\right]^{3 \times 3},
$$

where $\mathcal{Q}_{h}^{s}:\left[L^{2}\left(\Omega_{s}\right)\right]^{3} \rightarrow\left[\mathcal{S}_{h}^{0}\left(\Omega_{s}\right)\right]^{3}$ is the $\left[L^{2}\left(\Omega_{s}\right)\right]^{3}$-orthogonal projection. Actually, it turns out (see, e.g., [16, Theorem 3.16]) that $\Pi_{h}^{s}$ can be defined as a bounded linear operator from the larger space $\left[H^{\epsilon}\left(\Omega_{s}\right)\right]^{3 \times 3} \cap \mathbf{H}\left(\operatorname{div} ; \Omega_{s}\right)$ into $\mathcal{R} \mathcal{T}_{h}\left(\Omega_{s}\right)$ for all $\epsilon>0$, and that the following interpolation error estimate holds true:

$$
\left\|\boldsymbol{\tau}-\Pi_{h}^{s}(\boldsymbol{\tau})\right\|_{\left[L^{2}\left(\Omega_{s}\right)\right]^{3 \times 3}} \leq C h^{\min (1, \epsilon)}\left\{\|\boldsymbol{\tau}\|_{\left[H^{\epsilon}\left(\Omega_{s}\right)\right]^{3 \times 3}}+\|\operatorname{div}(\boldsymbol{\tau})\|_{\left[L^{2}\left(\Omega_{s}\right)\right]^{3}}\right\} .
$$

Next, for any $\delta \geq 0$ we introduce the Sobolev space

$$
\mathbf{H}^{\delta}\left(\operatorname{curl} ; \Omega_{m}\right):=\left\{\boldsymbol{W} \in\left[H^{\delta}\left(\Omega_{m}\right)\right]^{3}: \quad \operatorname{curl}(\boldsymbol{W}) \in\left[H^{\delta}\left(\Omega_{m}\right)\right]^{3}\right\}
$$

and endow it with its Hilbertian norm

$$
\|\boldsymbol{W}\|_{\mathbf{H}^{\delta}\left(\operatorname{curl} ; \Omega_{m}\right)}^{2}:=\|\boldsymbol{W}\|_{\left[H^{\delta}\left(\Omega_{m}\right)\right]^{3}}^{2}+\|\operatorname{curl}(\boldsymbol{W})\|_{\left[H^{\delta}\left(\Omega_{m}\right)\right]^{3}}^{2} .
$$

Then for any edge $E$ of $\mathcal{T}_{h}\left(\Omega_{m}\right)$ we denote by $\mathbf{t}_{E}$ a unit tangential vector along $E$. It follows from [2, Lemma 4.7] that if $\boldsymbol{W} \in \mathbf{H}^{\delta}\left(\operatorname{curl} ; \Omega_{m}\right)$ with $\delta>1 / 2$, then the moments $\int_{E} \boldsymbol{W} \cdot \mathbf{t}_{E}$ are meaningful. This guarantees that the interpolation operator $\Pi_{h}^{m}: \mathbf{H}^{\delta}\left(\mathbf{c u r l} ; \Omega_{m}\right) \rightarrow \mathbf{X}_{h}^{m}$ associated with the edge finite element, which is characterized by

$$
\int_{E} \Pi_{h}^{m}(\boldsymbol{W}) \cdot \mathbf{t}_{E}=\int_{E} \boldsymbol{W} \cdot \mathbf{t}_{E} \quad \forall \text { edges } E \text { of } \mathcal{T}_{h}\left(\Omega_{m}\right),
$$

is well defined and uniformly bounded. In addition, the following interpolation error estimate holds (see [1, Proposition 5.6]):

$$
\left\|\boldsymbol{W}-\Pi_{h}^{m}(\boldsymbol{W})\right\|_{\mathbf{H}\left(\mathbf{c u r l} ; \Omega_{m}\right)} \leq C h^{\delta}\|\boldsymbol{W}\|_{\mathbf{H}^{\delta}\left(\mathbf{c u r l} ; \Omega_{m}\right)}
$$

for all $\boldsymbol{W} \in \mathbf{H}^{\delta}\left(\operatorname{curl} ; \Omega_{m}\right)$ and for all $\delta \in(1 / 2,1]$. Another useful property of $\Pi_{h}^{m}$ is given by the following result.

Lemma 7.1. For each $\delta \in(1 / 2,1]$ define the space

$$
\boldsymbol{H}_{h}^{\delta}\left(\operatorname{curl} ; \Omega_{m}\right):=\left\{\boldsymbol{W} \in\left[H^{\delta}\left(\Omega_{m}\right)\right]^{3}: \quad \operatorname{curl}(\boldsymbol{W}) \in \operatorname{curl}\left(\mathbf{X}_{h}^{m}\right)\right\} .
$$

Then, the operator $\Pi_{h}^{m}$ is also well defined in $\boldsymbol{H}_{h}^{\delta}\left(\mathbf{c u r l} ; \Omega_{m}\right)$ and there exists a constant $C>0$, independent of $h$, such that

$$
\left\|\boldsymbol{W}-\Pi_{h}^{m}(\boldsymbol{W})\right\|_{\left[L^{2}\left(\Omega_{m}\right)\right]^{3}} \leq C h^{\delta}\|\boldsymbol{W}\|_{\left[H^{\delta}\left(\Omega_{m}\right)\right]^{3}} \quad \forall \boldsymbol{W} \in \boldsymbol{H}_{h}^{\delta}\left(\operatorname{curl} ; \Omega_{m}\right) .
$$

Proof. For the proof, see [16, Lemma 4.6]. 
Next, we need to introduce $\operatorname{curl}_{\Gamma}$-conforming surface finite elements on the manifold $\Gamma$. Actually, $\operatorname{div}_{\Gamma}$-conforming finite elements on manifolds are more frequently used in the literature since they arise naturally in the BEM-theory for Maxwell equations (see, e.g., [8] and the references therein). We still can benefit here from the result announced in the last reference for the Raviart-Thomas finite elements, since they may be translated to the bidimensional Nédélec finite elements by a simple $\pi / 2$ rotation in the space variable on each one of the faces compounding $\Gamma$. To be more specific, the lowest order bidimensional Nédélec finite element (also known as the rotated Raviart-Thomas finite element) approximation of the space

$$
\mathbf{H}\left(\operatorname{curl}_{\Gamma} ; \Gamma\right):=\left\{\boldsymbol{\varphi} \in \mathbf{L}_{\mathbf{t}}^{2}(\Gamma): \quad \operatorname{curl}_{\Gamma}(\boldsymbol{\varphi}) \in L^{2}(\Gamma)\right\},
$$

relative to the mesh $\mathcal{T}_{h}(\Gamma)$, is given by (see (6.2))

$$
\mathcal{N} \mathcal{D}_{h}(\Gamma):=\pi_{\mathbf{t}}\left(\mathbf{X}_{h}^{m}\right)
$$

The corresponding interpolation operator $\Pi_{h}^{\Gamma}: \mathbf{H}_{\|}^{\delta}(\Gamma) \cap \mathbf{H}\left(\operatorname{curl}_{\Gamma} ; \Gamma\right) \rightarrow \mathcal{N} \mathcal{D}_{h}(\Gamma)(\delta \in$ $(0,1])$ satisfies the following error estimate.

Lemma 7.2. For each $\delta \in(0,1]$ there exists a constant $C>0$, independent of $h$, such that

$$
\left\|\boldsymbol{\varphi}-\Pi_{h}^{\Gamma}(\boldsymbol{\varphi})\right\|_{\left[L^{2}(\Gamma)\right]^{3}} \leq C h^{\delta}\left\{\|\boldsymbol{\varphi}\|_{\mathbf{H}_{\|}^{\delta}(\Gamma)}+\left\|\operatorname{curl}_{\Gamma}(\boldsymbol{\varphi})\right\|_{L^{2}(\Gamma)}\right\}
$$

for all $\boldsymbol{\varphi} \in \mathbf{H}_{\|}^{\delta}(\Gamma) \cap \mathbf{H}\left(\operatorname{curl}_{\Gamma} ; \Gamma\right)$.

Proof. For the proof, see [8, Lemma 15].

For tangential vector fields with a discrete $\operatorname{curl}_{\Gamma}$, there holds the following variant.

Lemma 7.3. For each $\delta \in(0,1]$ there exists a constant $C>0$, independent of $h$, such that

$$
\left\|\boldsymbol{\varphi}-\Pi_{h}^{\Gamma}(\boldsymbol{\varphi})\right\|_{\left[L^{2}(\Gamma)\right]^{3}} \leq C h^{\delta}\|\boldsymbol{\varphi}\|_{\mathbf{H}_{\|}^{\delta}(\Gamma)} \quad \forall \boldsymbol{\varphi} \in \mathbf{H}_{\|}^{\delta}(\Gamma) \text { such that } \operatorname{curl}_{\Gamma}(\boldsymbol{\varphi}) \in \mathcal{S}_{h}^{0}(\Gamma) .
$$

Proof. For the proof, see [8, Lemma 16].

In this way, recalling the definition of the norm $\|\cdot\|_{\left.\mathbf{H}_{\Gamma} \text { (curl; } \Omega_{m}\right)}$ (see (4.1)) and using the commuting diagram property $\pi_{\mathbf{t}} \Pi_{h}^{m}=\Pi_{h}^{\Gamma} \boldsymbol{\pi}_{\mathbf{t}}$ together with (7.3) and Lemma 7.2 , we deduce that for each $\delta \in(1 / 2,1]$ there exists a constant $C>0$, independent of $h$, such that for all $\boldsymbol{W} \in \mathbf{H}^{\delta}\left(\operatorname{curl} ; \Omega_{m}\right)$ satisfying $\boldsymbol{\pi}_{\mathbf{t}}(\boldsymbol{W}) \in \mathbf{H}_{\|}^{\delta}(\Gamma) \cap$ $\mathbf{H}\left(\operatorname{curl}_{\Gamma} ; \Gamma\right)$ we have

$$
\begin{aligned}
\| \boldsymbol{W} & -\Pi_{h}^{m}(\boldsymbol{W}) \|_{\mathbf{H}_{\Gamma}\left(\mathbf{c u r l} ; \Omega_{m}\right)} \\
& :=\left\{\left\|\boldsymbol{W}-\Pi_{h}^{m}(\boldsymbol{W})\right\|_{\mathbf{H}\left(\mathbf{c u r l} ; \Omega_{m}\right)}^{2}+\left\|\boldsymbol{\pi}_{\mathbf{t}}\left(\boldsymbol{W}-\Pi_{h}^{m}(\boldsymbol{W})\right)\right\|_{\left[L^{2}(\Gamma)\right]^{3}}^{2}\right\}^{1 / 2} \\
& \leq C h^{\delta}\left\{\|\boldsymbol{W}\|_{\mathbf{H}^{\delta}\left(\mathbf{c u r l} ; \Omega_{m}\right)}+\left\|\boldsymbol{\pi}_{\mathbf{t}}(\boldsymbol{W})\right\|_{\mathbf{H}_{\|}^{\delta}(\Gamma)}+\left\|\operatorname{curl}_{\Gamma}\left(\boldsymbol{\pi}_{\mathbf{t}}(\boldsymbol{W})\right)\right\|_{L^{2}(\Gamma)}\right\},
\end{aligned}
$$

which constitutes an approximation property of the space $\mathbf{X}_{h}^{m}$. The corresponding properties of the remaining finite element subspaces are established as follows (see, for instance, [21]):

$\left(\mathrm{AP}_{h}\right)$ For each $\epsilon \in(0,1]$ and for each $\boldsymbol{\tau} \in\left[H^{\epsilon}\left(\Omega_{s}\right)\right]^{3 \times 3}$, with $\operatorname{div}(\boldsymbol{\tau}) \in\left[H^{\epsilon}\left(\Omega_{s}\right)\right]^{3}$,

$$
\left\|\boldsymbol{\tau}-\Pi_{h}^{s}(\boldsymbol{\tau})\right\|_{\mathbf{H}\left(\operatorname{div} ; \Omega_{s}\right)} \leq C h^{\epsilon}\left\{\|\boldsymbol{\tau}\|_{\left[H^{\epsilon}\left(\Omega_{s}\right)\right]^{3 \times 3}}+\|\operatorname{div}(\boldsymbol{\tau})\|_{\left[H^{\epsilon}\left(\Omega_{s}\right)\right]^{3}}\right\} .
$$


$\left(\operatorname{AP}_{\bar{h}}^{\psi}\right)$ For each $\epsilon \in(1 / 2,3 / 2]$ and for each $\varphi \in\left[H^{\epsilon}(\Sigma)\right]^{3}$,

$$
\inf _{\boldsymbol{\varphi}_{h} \in \mathrm{M}_{\tilde{h}}}\left\|\boldsymbol{\varphi}-\boldsymbol{\varphi}_{h}\right\|_{\left[H^{1 / 2}(\Sigma)\right]^{3}} \leq C \tilde{h}^{\epsilon-1 / 2}\|\boldsymbol{\varphi}\|_{\left[H^{\epsilon}(\Sigma)\right]^{3}} .
$$

$\left(\mathrm{AP}_{h}^{r}\right)$ For each $\epsilon \in[0,1]$ and for each $\boldsymbol{r} \in\left[H^{\epsilon}\left(\Omega_{s}\right)\right]^{3 \times 3} \cap\left[L^{2}\left(\Omega_{s}\right)\right]_{\mathrm{asym}}^{3 \times 3}$,

$$
\inf _{\boldsymbol{r}_{h} \in \mathbf{M}_{h}}\left\|\boldsymbol{r}-\boldsymbol{r}_{h}\right\|_{\left[L^{2}\left(\Omega_{s}\right)\right]^{3 \times 3}} \leq C h^{\epsilon}\|\boldsymbol{r}\|_{\left[H^{\epsilon}\left(\Omega_{s}\right)\right]^{3 \times 3}} .
$$

$\left(\mathrm{AP}_{h}^{\boldsymbol{u}}\right)$ For each $\epsilon \in[0,1]$ and for each $\boldsymbol{v} \in\left[H^{\epsilon}\left(\Omega_{s}\right)\right]^{3}$,

$$
\left\|\boldsymbol{v}-\mathcal{Q}_{h}^{s}(\boldsymbol{v})\right\|_{\left[L^{2}\left(\Omega_{s}\right)\right]^{3}} \leq C h^{\epsilon}\|\boldsymbol{v}\|_{\left[H^{\epsilon}\left(\Omega_{s}\right)\right]^{3}} .
$$

Note that $\left(\mathrm{AP}_{h}^{\boldsymbol{\sigma}}\right)$ is actually a straightforward consequence of (7.1), (7.2), and $\left(\mathrm{AP}_{h}^{u}\right)$

We end this section with the following inverse inequality.

Lemma 7.4. Assume that the family of triangulations $\mathcal{T}_{h}(\Sigma)$ is quasi-uniform. Then, for each $\delta \in[0,1)$ there exists a constant $C>0$, independent of $h$, such that

$$
\|\varphi\|_{H^{1 / 2+\delta}(\Sigma)} \leq C h^{-\delta}\|\varphi\|_{H^{1 / 2}(\Sigma)} \quad \forall \varphi \in \mathcal{S}_{h}^{1}(\Sigma)
$$

Proof. This result follows easily by combining known results (cf. Lemma 5.57 and Remark 5.58 in [21]) with classical interpolation results. We omit further details here.

7.2. Well-posedness of the discrete problem. In this section we prove the well-posedness of the discrete problem (6.4). For this purpose, according to a classical result on projection methods for compact perturbations of invertible operators (see, e.g., Theorem 13.7 in [18]), it suffices to show that the Galerkin scheme associated with the isomorphism $\left(\begin{array}{cc}\mathbb{A}_{0} & \mathbb{B}_{0}^{*} \\ \mathbb{B}_{0} & \mathbf{0}\end{array}\right)$ is well-posed. Hence, in what follows we prove that $\mathbf{A}_{0}$ and $\mathbf{B}_{0}$ satisfy the corresponding inf-sup conditions on the finite element subspace $\mathbb{X}_{h} \times \mathbb{M}_{\tilde{h}, h}$, thus providing the discrete analogues of Lemmas 5.4 and 5.5.

LEMma 7.5. There exist $\left.C_{0} \in\right] 0,1[$ and $\beta>0$, independent of $h$ and $\tilde{h}$, such that for all $h \leq C_{0} \tilde{h}$ and for each $(\boldsymbol{\psi}, \boldsymbol{r}) \in \mathbb{M}_{\tilde{h}, h}$ we have

$$
\sup _{(\boldsymbol{W}, \boldsymbol{\tau}) \in \mathbb{X}_{h} \backslash\{\mathbf{0}\}} \frac{\left|\mathbf{B}_{0}((\boldsymbol{W}, \boldsymbol{\tau}),(\boldsymbol{\psi}, \boldsymbol{r}))\right|}{\|(\boldsymbol{W}, \boldsymbol{\tau})\|_{\mathbb{X}}} \geq \beta\|(\boldsymbol{\psi}, \boldsymbol{r})\|_{\mathbb{M}} .
$$

Proof. The proof results from a slight adjustment of that given in [12] for the 2D case. Indeed, applying [19, Theorem 4.5], as done in [12, Lemma 5.1], we deduce the existence of $C_{1}>0$, independent of $h$ and $\tilde{h}$, such that for each $(\boldsymbol{\psi}, \boldsymbol{r}) \in \mathbb{M}_{\tilde{h}, h}$ we have

$$
\sup _{(\boldsymbol{W}, \boldsymbol{\tau}) \in \mathbb{X}_{h} \backslash\{\mathbf{0}\}} \frac{\left|\mathbf{B}_{0}((\boldsymbol{W}, \boldsymbol{\tau}),(\boldsymbol{\psi}, \boldsymbol{r}))\right|}{\|(\boldsymbol{W}, \boldsymbol{\tau})\|_{\mathbb{X}}} \geq C_{1}\|\boldsymbol{r}\|_{\left[L^{2}\left(\Omega_{s}\right)\right]^{3 \times 3}} .
$$

Now, according to the uniform extension provided in [22, Theorem 4.1.9], there exist a linear operator $\mathcal{E}_{h}:\left[\mathcal{S}_{h}^{0}(\Sigma)\right]^{3} \rightarrow \mathbf{X}_{h}^{s}$ and a constant $C_{2}>0$, independent of $h$, such that

$$
\gamma_{\boldsymbol{n}}\left(\mathcal{E}_{h}\left(\boldsymbol{\xi}_{h}\right)\right)=\boldsymbol{\xi}_{h} \quad \text { on } \Sigma \quad \text { and } \quad\left\|\mathcal{E}_{h}\left(\boldsymbol{\xi}_{h}\right)\right\|_{\mathbf{H}\left(\mathrm{div} ; \Omega_{s}\right)} \leq C_{2}\left\|\boldsymbol{\xi}_{h}\right\|_{\left[H^{-1 / 2}(\Sigma)\right]^{3}}
$$


for all $\boldsymbol{\xi}_{h} \in\left[\mathcal{S}_{h}^{0}(\Sigma)\right]^{3}$. Hence, recalling the definition of $\mathbf{B}_{0}$ (cf. (5.24)), it follows that

$$
\begin{aligned}
& \sup _{(\boldsymbol{W}, \boldsymbol{\tau}) \in \mathbb{X}_{h} \backslash\{\mathbf{0}\}} \frac{\left|\mathbf{B}_{0}((\boldsymbol{W}, \boldsymbol{\tau}),(\boldsymbol{\psi}, \boldsymbol{r}))\right|}{\|(\boldsymbol{W}, \boldsymbol{\tau})\|_{\mathbf{X}}} \geq \frac{\left|\mathbf{B}_{0}\left(\left(\mathbf{0}, \mathcal{E}_{h}\left(\boldsymbol{\xi}_{h}\right)\right),(\boldsymbol{\psi}, \boldsymbol{r})\right)\right|}{\left\|\mathcal{E}_{h}\left(\boldsymbol{\xi}_{h}\right)\right\|_{\mathbf{H}\left(\mathbf{d i v} ; \Omega_{s}\right)}} \\
& \geq \frac{k^{2}}{C_{2}} \frac{\left|\left\langle\boldsymbol{\xi}_{h}, \boldsymbol{\psi}\right\rangle_{\Sigma}\right|}{\left\|\boldsymbol{\xi}_{h}\right\|_{\left[H^{-1 / 2}(\Sigma)\right]^{3}}}-k^{2}\|\boldsymbol{r}\|_{\left[L^{2}\left(\Omega_{s}\right)\right]^{3 \times 3}} \quad \forall \boldsymbol{\xi}_{h} \in\left[\mathcal{S}_{h}^{0}(\Sigma)\right]^{3} \backslash\{\boldsymbol{0}\} .
\end{aligned}
$$

Now, given $\boldsymbol{\psi} \in \mathbf{M}_{\tilde{h}} \backslash\{\mathbf{0}\}:=\left[\mathcal{S}_{\tilde{h}}^{1}(\Sigma)\right]^{3} \backslash\{\mathbf{0}\}$, we let $\boldsymbol{z} \in\left[H^{1}\left(\Omega_{s}\right)\right]^{3}$ be the unique solution of the problem

$$
-\Delta \boldsymbol{z}+\boldsymbol{z}=\mathbf{0} \quad \text { in } \Omega_{s}, \quad \boldsymbol{z}=\boldsymbol{\psi} \text { on } \Sigma .
$$

Notice that the corresponding continuous dependence result gives

$$
\|\boldsymbol{z}\|_{\left[H^{1}\left(\Omega_{s}\right)\right]^{3}} \leq c\|\boldsymbol{\psi}\|_{\left[H^{1 / 2}(\Sigma)\right]^{3}},
$$

and, by virtue of the trace theorem and Green's formula, we also have that

$$
C_{3}\|\boldsymbol{\psi}\|_{\left[H^{1 / 2}(\Sigma)\right]^{3}}^{2} \leq\|\boldsymbol{z}\|_{\left[H^{1}\left(\Omega_{s}\right)\right]^{3}}^{2}=\left\langle\boldsymbol{\gamma}_{\boldsymbol{n}}(\nabla \boldsymbol{z}), \boldsymbol{\psi}\right\rangle_{\Sigma}
$$

Moreover, since $\boldsymbol{\psi}$ is a piecewise-polynomial continuous function on $\Sigma$ and $\Omega_{s}$ is a Lipschitz polyhedral domain, the solution $\boldsymbol{z}$ belongs to the Sobolev space $\left[H^{1+\delta}\left(\Omega_{s}\right)\right]^{3}$ for a suitable $\delta \in(1 / 2,1)$ (see [11]), and the following estimate holds true:

$$
\|\boldsymbol{z}\|_{\left[H^{1+\delta}\left(\Omega_{s}\right)\right]^{3}} \leq C_{4}\|\boldsymbol{\psi}\|_{\left[H^{1 / 2+\delta}(\Sigma)\right]^{3}} .
$$

We now let $\boldsymbol{\xi}_{h}^{*}:=\gamma_{\boldsymbol{n}}\left(\Pi_{h}^{s}(\nabla \boldsymbol{z})\right)$. Then, applying the boundedness of the normal trace operator (2.1), the approximation property $\left(\mathrm{AP}_{h}^{\boldsymbol{\sigma}}\right)$, the fact that $\operatorname{div}(\nabla \boldsymbol{z})=$ $\Delta \boldsymbol{z}=\boldsymbol{z}$ in $\Omega_{s}$, the regularity estimate (7.12), and the inverse inequality provided by Lemma 7.4, we deduce that

$$
\begin{aligned}
& \left\|\boldsymbol{\gamma}_{\boldsymbol{n}}(\nabla \boldsymbol{z})-\boldsymbol{\xi}_{h}^{*}\right\|_{\left[H^{-1 / 2}(\Sigma)\right]^{3}} \\
& \quad \leq C\left\|\nabla \boldsymbol{z}-\Pi_{h}^{s}(\nabla \boldsymbol{z})\right\|_{\mathbf{H}\left(\operatorname{div} ; \Omega_{s}\right)} \leq C_{5} h^{\delta}\left\{\|\nabla \boldsymbol{z}\|_{\left[H^{\delta}\left(\Omega_{s}\right)\right]^{3 \times 3}}+\|\boldsymbol{z}\|_{\left[H^{\delta}\left(\Omega_{s}\right)\right]^{3}}\right\} \\
& \quad \leq C_{6} h^{\delta}\|\boldsymbol{z}\|_{\left[H^{1+\delta}\left(\Omega_{s}\right)\right]^{3}} \leq C_{4} C_{6} h^{\delta}\|\boldsymbol{\psi}\|_{\left[H^{1 / 2+\delta}(\Sigma)\right]^{3}} \leq C_{7}\left(\frac{h}{\tilde{h}}\right)^{\delta}\|\boldsymbol{\psi}\|_{\left[H^{1 / 2}(\Sigma)\right]^{3}},
\end{aligned}
$$

which, together with the inequality

$$
\begin{gathered}
\left\|\boldsymbol{\xi}_{h}^{*}\right\|_{\left[H^{-1 / 2}(\Sigma)\right]^{3}} \leq\left\|\boldsymbol{\gamma}_{\boldsymbol{n}}(\nabla \boldsymbol{z})-\boldsymbol{\xi}_{h}^{*}\right\|_{\left[H^{-1 / 2}(\Sigma)\right]^{3}}+\left\|\boldsymbol{\gamma}_{\boldsymbol{n}}(\nabla \boldsymbol{z})\right\|_{\left[H^{-1 / 2}(\Sigma)\right]^{3}} \\
\leq\left\|\boldsymbol{\gamma}_{\boldsymbol{n}}(\nabla \boldsymbol{z})-\boldsymbol{\xi}_{h}^{*}\right\|_{\left[H^{-1 / 2}(\Sigma)\right]^{3}}+c\|\nabla \boldsymbol{z}\|_{\mathbf{H}\left(\mathbf{d i v} ; \Omega_{s}\right)},
\end{gathered}
$$

the fact that $\|\nabla \boldsymbol{z}\|_{\mathbf{H}\left(\mathbf{d i v} ; \Omega_{s}\right)}=\|\boldsymbol{z}\|_{\left[H^{1}\left(\Omega_{s}\right)\right]^{3}}$, and the estimate (7.10), implies

$$
\left\|\boldsymbol{\xi}_{h}^{*}\right\|_{\left[H^{-1 / 2}(\Sigma)\right]^{3}} \leq C_{8}\|\boldsymbol{\psi}\|_{\left[H^{1 / 2}(\Sigma)\right]^{3}} \quad \forall h \leq \tilde{h} .
$$

It follows, using (7.11) and the above estimates, that

$$
\begin{aligned}
\left\langle\boldsymbol{\xi}_{h}^{*}, \boldsymbol{\psi}\right\rangle_{\Sigma}=\left\langle\boldsymbol{\gamma}_{\boldsymbol{n}}(\nabla \boldsymbol{z}), \boldsymbol{\psi}\right\rangle_{\Sigma}-\left\langle\boldsymbol{\gamma}_{\boldsymbol{n}}(\nabla \boldsymbol{z})-\boldsymbol{\xi}_{h}^{*}, \boldsymbol{\psi}\right\rangle_{\Sigma} \\
\quad \geq\left\{C_{3}-C_{7}\left(\frac{h}{\tilde{h}}\right)^{\delta}\right\}\|\boldsymbol{\psi}\|_{\left[H^{1 / 2}(\Sigma)\right]^{3}}^{2} \\
\quad \geq\left\{\frac{C_{3}}{C_{8}}-\frac{C_{7}}{C_{8}}\left(\frac{h}{\tilde{h}}\right)^{\delta}\right\}\|\boldsymbol{\psi}\|_{\left[H^{1 / 2}(\Sigma)\right]^{3}}\left\|\boldsymbol{\xi}_{h}^{*}\right\|_{\left[H^{-1 / 2}(\Sigma)\right]^{3}} \quad \forall h \leq \tilde{h} .
\end{aligned}
$$

Copyright (c) by SIAM. Unauthorized reproduction of this article is prohibited. 
In this way, substituting in particular $\boldsymbol{\xi}_{h}^{*}$ into (7.9) and taking $h \leq C_{0} \tilde{h}$, with $C_{0}:=$ $\min \left\{1,\left(\frac{C_{3}}{2 C_{7}}\right)^{1 / \delta}\right\}$, we deduce that

$$
\sup _{(\boldsymbol{W}, \boldsymbol{\tau}) \in \mathbb{X}_{h} \backslash\{\mathbf{0}\}} \frac{\left|\mathbf{B}_{0}((\boldsymbol{W}, \boldsymbol{\tau}),(\boldsymbol{\psi}, \boldsymbol{r}))\right|}{\|(\boldsymbol{W}, \boldsymbol{\tau})\| \mathbf{X}} \geq \frac{k^{2} C_{3}}{2 C_{2} C_{8}}\|\boldsymbol{\psi}\|_{\left[H^{1 / 2}\left(\Omega_{s}\right)\right]^{3}}-k^{2}\|\boldsymbol{r}\|_{\left[L^{2}\left(\Omega_{s}\right)\right]^{3 \times 3}} .
$$

Finally, a judicious combination of the above inequality with (7.8) implies the required discrete inf-sup condition (7.7), thus completing the proof.

At this point we remark that the technical requirement arising from the above proof, namely the condition $h \leq C_{0} \tilde{h}$, is the only reason for choosing different meshes in the domain and on $\Sigma$. Further remarks on this matter are provided in section 8 .

In what follows, and in order to establish later on the discrete inf-sup condition for $\mathbf{A}_{0}$, we define discrete versions of the operators $\mathcal{P}_{s}$ and $\mathcal{P}_{m}$. To this end, we first introduce finite element subspaces of $\mathbb{H}$ and $\mathbb{Q}$ (cf. (5.9), (5.10)), respectively,

$$
\mathbb{H}_{h}:=\left\{\boldsymbol{\tau} \in \mathbf{X}_{h}^{s}: \quad \boldsymbol{\tau} \boldsymbol{n}=\mathbf{0} \text { on } \Sigma\right\}
$$

and

$$
\mathbb{Q}_{h}:=\left\{\left[\mathcal{S}_{h}^{0}\left(\Omega_{s}\right)\right]^{3} \cap \mathbb{R M}\left(\Omega_{s}\right)^{\perp}\right\} \times \mathbf{M}_{h}
$$

where $\mathbb{R M}\left(\Omega_{s}\right)$ is the space of rigid body motions (cf. (5.5)). Then, given $\tau \in$ $\mathbf{H}\left(\operatorname{div} ; \Omega_{s}\right)$, we consider the following Galerkin approximation of (5.8): Find the triple $\left(\tilde{\boldsymbol{\sigma}}_{h},\left(\tilde{\boldsymbol{u}}_{h}, \tilde{\boldsymbol{r}}_{h}\right)\right) \in \mathbb{H}_{h} \times \mathbb{Q}_{h}$ such that

$$
\begin{aligned}
\int_{\Omega_{s}} \mathcal{C}^{-1} \tilde{\boldsymbol{\sigma}}_{h}: \tilde{\boldsymbol{\tau}}+ & \int_{\Omega_{s}}\left\{\tilde{\boldsymbol{u}}_{h} \cdot \operatorname{div}(\tilde{\boldsymbol{\tau}})+\tilde{\boldsymbol{r}}_{h}: \tilde{\boldsymbol{\tau}}\right\}=0 \\
& \int_{\Omega_{s}}\left\{\tilde{\boldsymbol{v}} \cdot \operatorname{div}\left(\tilde{\boldsymbol{\sigma}}_{h}\right)+\tilde{\boldsymbol{s}}: \tilde{\boldsymbol{\sigma}}_{h}\right\}=\int_{\Omega_{s}} \tilde{\boldsymbol{v}} \cdot(\mathcal{I}-\mathbf{M})(\operatorname{div}(\boldsymbol{\tau}))
\end{aligned}
$$

for all $(\tilde{\boldsymbol{\tau}},(\tilde{\boldsymbol{v}}, \tilde{\boldsymbol{s}})) \in \mathbb{H}_{h} \times \mathbb{Q}_{h}$. Hence, proceeding analogously to the 2D case (see section 4.3 in [13]), and noting that the corresponding Neumann boundary condition involved in (5.8) and (7.13) is homogeneous, we can show that there exists a unique $\left(\tilde{\boldsymbol{\sigma}}_{h},\left(\tilde{\boldsymbol{u}}_{h}, \tilde{\boldsymbol{r}}_{h}\right)\right) \in \mathbb{H}_{h} \times \mathbb{Q}_{h}$ solution of (7.13). Moreover, there exist $C, \tilde{C}>0$, independent of $h$, such that

$$
\left\|\tilde{\boldsymbol{\sigma}}_{h}\right\|_{\mathbf{H}\left(\operatorname{div} ; \Omega_{s}\right)}+\left\|\tilde{\boldsymbol{u}}_{h}\right\|_{\left[L^{2}\left(\Omega_{s}\right)\right]^{3}}+\left\|\tilde{\boldsymbol{r}}_{h}\right\|_{\left[L^{2}\left(\Omega_{s}\right)\right]^{3 \times 3}} \leq C\|\operatorname{div}(\boldsymbol{\tau})\|_{\left[L^{2}\left(\Omega_{s}\right)\right]^{3}}
$$

and

$$
\begin{aligned}
\left\|\tilde{\boldsymbol{\sigma}}-\tilde{\boldsymbol{\sigma}}_{h}\right\|_{\mathbf{H}\left(\mathbf{d i v} ; \Omega_{s}\right)}+\left\|\tilde{\boldsymbol{u}}-\tilde{\boldsymbol{u}}_{h}\right\|_{\left[L^{2}\left(\Omega_{s}\right)\right]^{3}}+\left\|\tilde{\boldsymbol{r}}-\tilde{\boldsymbol{r}}_{h}\right\|_{\left[L^{2}\left(\Omega_{s}\right)\right]^{3 \times 3}} \\
\leq \tilde{C}\left\{\left\|\left(\mathcal{I}-\Pi_{h}^{s}\right)(\tilde{\boldsymbol{\sigma}})\right\|_{\mathbf{H}\left(\operatorname{div} ; \Omega_{s}\right)}+\left\|\left(\mathcal{I}-\mathcal{Q}_{h}^{s}\right)(\tilde{\boldsymbol{u}})\right\|_{\left[L^{2}\left(\Omega_{s}\right)\right]^{3}}\right. \\
\left.\quad+\inf _{\tilde{\boldsymbol{s}}_{h} \in \mathbf{M}_{h}}\left\|\tilde{\boldsymbol{r}}-\tilde{\boldsymbol{s}}_{h}\right\|_{\left[L^{2}\left(\Omega_{s}\right)\right]^{3 \times 3}}\right\},
\end{aligned}
$$

where $(\tilde{\boldsymbol{\sigma}},(\tilde{\boldsymbol{u}}, \tilde{\boldsymbol{r}})) \in \mathbb{H} \times \mathbb{Q}$ is the unique solution of $(5.8)$ and $\Pi_{h}^{s}:\left[H^{1}\left(\Omega_{s}\right)\right]^{3 \times 3} \rightarrow$ $\mathcal{R} \mathcal{T}_{h}\left(\Omega_{s}\right)$ and $\mathcal{Q}_{h}^{s}:\left[L^{2}\left(\Omega_{s}\right)\right]^{3} \rightarrow\left[\mathcal{S}_{h}^{0}\left(\Omega_{s}\right)\right]^{3}$ are the operators defined at the beginning of section 7.1. 
By virtue of the previous analysis, we are now in a position to introduce a discrete version of the operator $\mathcal{P}_{s}$ (cf. (5.12)). In fact, having in mind that $\left(\tilde{\boldsymbol{\sigma}}_{h},\left(\tilde{\boldsymbol{u}}_{h}, \tilde{\boldsymbol{r}}_{h}\right)\right) \in$ $\mathbb{H}_{h} \times \mathbb{Q}_{h}$ is the unique solution of (7.13), we define

$$
\begin{aligned}
\mathcal{P}_{s, h}: \mathbf{H}\left(\operatorname{div} ; \Omega_{s}\right) & \rightarrow \mathbb{H}_{h} \subseteq \mathbf{X}_{h}^{s}, \\
\boldsymbol{\tau} & \rightarrow \mathcal{P}_{s, h}(\boldsymbol{\tau}):=\tilde{\boldsymbol{\sigma}}_{h} .
\end{aligned}
$$

It follows from (7.13) and (7.14) that $\mathcal{P}_{s, h}$ is a bounded linear operator. In addition, it is clear from the definition of $\mathbb{H}_{h}$ and (7.13) that

$$
\mathcal{P}_{s, h}(\boldsymbol{\tau}) \boldsymbol{n}=\mathbf{0} \quad \text { on } \Sigma \quad \text { and } \quad \int_{\Omega_{s}} \mathcal{P}_{s, h}(\boldsymbol{\tau}): \tilde{\boldsymbol{s}}=0 \quad \forall \tilde{\boldsymbol{s}} \in \mathbf{M}_{h}
$$

Next, as a straightforward consequence of the error estimate (7.15) and the approximation properties provided in section 7.1, we are able to establish the following result.

Lemma 7.6. Let $\epsilon>0$ be the parameter defining the regularity of the solution of (5.6). Then, there exists $C>0$, independent of $h$, such that

$$
\left\|\mathcal{P}_{s}\left(\boldsymbol{\tau}_{h}\right)-\mathcal{P}_{s, h}\left(\boldsymbol{\tau}_{h}\right)\right\|_{\mathbf{H}\left(\operatorname{div} ; \Omega_{s}\right)} \leq C h^{\epsilon}\left\|\operatorname{div}\left(\boldsymbol{\tau}_{h}\right)\right\|_{\left[L^{2}\left(\Omega_{s}\right)\right]^{3}} \quad \forall \boldsymbol{\tau}_{h} \in \mathbf{X}_{h}^{s} .
$$

Proof. The proof follows similarly to the proof of Lemma 5.4 in [12]. We omit further details here.

On the other hand, we now let $\tilde{\Pi}_{h}^{m}$ be the lowest order Raviart-Thomas interpolation operator associated with the triangulation $\mathcal{T}_{h}\left(\Omega_{m}\right)$. It follows, using the well-known commuting diagram property

$$
\operatorname{curl} \Pi_{h}^{m}=\tilde{\Pi}_{h}^{m} \operatorname{curl},
$$

that for each $\boldsymbol{W} \in \mathbf{X}_{h}^{m}$ we have

$$
\begin{aligned}
& \operatorname{curl}\left(\mathcal{P}_{m}(\boldsymbol{W})\right)=\tilde{\Pi}_{h}^{m}\left\{\operatorname{curl}\left(\mathcal{P}_{m}(\boldsymbol{W})\right)\right\}=\tilde{\Pi}_{h}^{m}\{\operatorname{curl}(\boldsymbol{W})\} \\
& \quad=\operatorname{curl}\left\{\Pi_{h}^{m}(\boldsymbol{W})\right\} \in \operatorname{curl}\left(\mathbf{X}_{h}^{m}\right),
\end{aligned}
$$

which, recalling that $\mathcal{P}_{m}(\boldsymbol{W}) \in\left[H^{1}\left(\Omega_{m}\right)\right]^{3}$, shows that $\mathcal{P}_{m}(\boldsymbol{W})$ belongs to the space $\boldsymbol{H}_{h}^{1}\left(\operatorname{curl} ; \Omega_{m}\right)$ (cf. (7.4) with $\delta=1$ ). In this way, Lemma 7.1 implies that $\Pi_{h}^{m}$ can be applied to $\mathcal{P}_{m}(\boldsymbol{W})$, and hence we define the discrete version of the operator $\mathcal{P}_{m}$ as follows:

$$
\begin{aligned}
\mathcal{P}_{m, h}: \mathbf{X}_{h}^{m} & \rightarrow \mathbf{X}_{h}^{m}, \\
\boldsymbol{W} & \mapsto \mathcal{P}_{m . h}(\boldsymbol{W}):=\Pi_{h}^{m}\left(\mathcal{P}_{m}(\boldsymbol{W})\right) .
\end{aligned}
$$

Lemma 7.7. There exists a constant $C>0$, independent of $h$, such that

$$
\left\|\mathcal{P}_{m}(\boldsymbol{W})-\mathcal{P}_{m, h}(\boldsymbol{W})\right\|_{\mathbf{H}_{\Gamma}\left(\mathbf{c u r l} ; \Omega_{m}\right)} \leq C h^{1 / 2}\|\boldsymbol{W}\|_{\mathbf{H}_{\Gamma}\left(\mathbf{c u r l} ; \Omega_{m}\right)} \quad \forall \boldsymbol{W} \in \mathbf{X}_{h}^{m} .
$$

Proof. It is easy to see that $\operatorname{curl}\left(\mathcal{P}_{m}(\boldsymbol{W})\right)=\operatorname{curl}\left(\mathcal{P}_{m, h}(\boldsymbol{W})\right)$, which yields

$$
\left\|\mathcal{P}_{m}(\boldsymbol{W})-\mathcal{P}_{m, h}(\boldsymbol{W})\right\|_{\mathbf{H}\left(\operatorname{curl} ; \Omega_{m}\right)}=\left\|\mathcal{P}_{m}(\boldsymbol{W})-\mathcal{P}_{m, h}(\boldsymbol{W})\right\|_{\left[L^{2}\left(\Omega_{m}\right)\right]^{3}},
$$

and hence, applying Lemma 7.1 (cf. (7.5)) and the boundedness of $\mathcal{P}_{m}: \mathbf{H}_{\Gamma}\left(\mathbf{c u r l} ; \Omega_{m}\right)$ $\rightarrow\left[H^{1}\left(\Omega_{m}\right)\right]^{3}$, we deduce that for each $\boldsymbol{W} \in \mathbf{X}_{h}^{m}$ there holds

$$
\left\|\mathcal{P}_{m}(\boldsymbol{W})-\mathcal{P}_{m, h}(\boldsymbol{W})\right\|_{\mathbf{H}\left(\mathbf{c u r l} ; \Omega_{m}\right)} \leq C h\left\|\mathcal{P}_{m}(\boldsymbol{W})\right\|_{\left[H^{1}\left(\Omega_{m}\right)\right]^{3}} \leq C h\|\boldsymbol{W}\|_{\mathbf{H}_{\Gamma}\left(\mathbf{c u r l} ; \Omega_{m}\right)} .
$$


On the other hand, using the commuting diagram property $\boldsymbol{\pi}_{\mathbf{t}} \Pi_{h}^{m}=\Pi_{h}^{\Gamma} \boldsymbol{\pi}_{\mathbf{t}}$, we have that

$$
\boldsymbol{\pi}_{\mathbf{t}}\left(\mathcal{P}_{m, h}(\boldsymbol{W})\right)=\boldsymbol{\pi}_{\mathbf{t}}\left(\Pi_{h}^{m}\left(\mathcal{P}_{m}(\boldsymbol{W})\right)\right)=\Pi_{h}^{\Gamma}\left(\boldsymbol{\pi}_{\mathbf{t}}\left(\mathcal{P}_{m}(\boldsymbol{W})\right)\right)
$$

In addition, since $\operatorname{curl}_{\Gamma} \boldsymbol{\pi}_{\mathbf{t}}=\operatorname{div}_{\Gamma} \boldsymbol{\gamma}_{\mathbf{t}}$ and $\operatorname{div}_{\Gamma}\left(\boldsymbol{\gamma}_{\mathbf{t}}(\boldsymbol{W})\right)=\gamma_{\boldsymbol{n}}(\boldsymbol{\operatorname { c u r l }}(\boldsymbol{W})) \in H^{-1 / 2}(\Gamma)$ for each $\boldsymbol{W} \in \mathbf{H}\left(\operatorname{curl} ; \Omega_{m}\right)$ (see [7]), we deduce that for each $\boldsymbol{W} \in \mathbf{X}_{h}^{m}$ there holds

$$
\begin{aligned}
\operatorname{curl}_{\Gamma}\left(\boldsymbol{\pi}_{\mathbf{t}}\left(\mathcal{P}_{m}(\boldsymbol{W})\right)\right)=\operatorname{div}_{\Gamma}\left(\boldsymbol{\gamma}_{\mathbf{t}}\left(\mathcal{P}_{m}(\boldsymbol{W})\right)\right)=\gamma_{\boldsymbol{n}}\left(\operatorname{curl}\left(\mathcal{P}_{m}(\boldsymbol{W})\right)\right) \\
=\gamma_{\boldsymbol{n}}(\operatorname{curl}(\boldsymbol{W})) \in \mathcal{S}_{h}^{0}(\Gamma) .
\end{aligned}
$$

Consequently, applying now the boundedness of the operators $\mathcal{P}_{m}: \mathbf{H}_{\Gamma}\left(\mathbf{c u r l} ; \Omega_{m}\right) \rightarrow$ $\left[H^{1}\left(\Omega_{m}\right)\right]^{3}$ and $\boldsymbol{\pi}_{\mathbf{t}}:\left[H^{1}\left(\Omega_{m}\right)\right]^{3} \rightarrow \mathbf{H}_{\|}^{1 / 2}(\Gamma)$ and the estimate provided by Lemma 7.3 , we find that

$$
\begin{aligned}
& \left\|\boldsymbol{\pi}_{\mathbf{t}}\left(\mathcal{P}_{m}(\boldsymbol{W})-\mathcal{P}_{m, h}(\boldsymbol{W})\right)\right\|_{\left[L^{2}(\Gamma)\right]^{3}}=\left\|\boldsymbol{\pi}_{\mathbf{t}}\left(\mathcal{P}_{m}(\boldsymbol{W})\right)-\Pi_{h}^{\Gamma}\left(\boldsymbol{\pi}_{\mathbf{t}}\left(\mathcal{P}_{m}(\boldsymbol{W})\right)\right)\right\|_{\left[L^{2}(\Gamma)\right]^{3}} \\
& \quad \leq C h^{1 / 2}\left\|\boldsymbol{\pi}_{\mathbf{t}}\left(\mathcal{P}_{m}(\boldsymbol{W})\right)\right\|_{\mathbf{H}_{\|}^{1 / 2}(\Gamma)} \leq C h^{1 / 2}\left\|\mathcal{P}_{m}(\boldsymbol{W})\right\|_{\left[H^{1}\left(\Omega_{m}\right)\right]^{3}} \\
& \quad \leq C h^{1 / 2}\|\boldsymbol{W}\|_{\mathbf{H}_{\Gamma}\left(\mathbf{c u r l} ; \Omega_{m}\right)},
\end{aligned}
$$

which, together with (7.20), yields the required estimate. The proof is now complete.

We are now ready to prove the discrete weak coercivity of $\mathbf{A}_{0}$. To this end, we let $\mathbb{V}_{\tilde{h}, h}$ be the discrete kernel of $\mathbf{B}_{0}$, that is,

$$
\mathbb{V}_{\tilde{h}, h}:=\left\{(\boldsymbol{W}, \boldsymbol{\tau}) \in \mathbb{X}_{h}: \quad \mathbf{B}_{0}((\boldsymbol{W}, \boldsymbol{\tau}),(\boldsymbol{\varphi}, \boldsymbol{s}))=0 \forall(\boldsymbol{\varphi}, \boldsymbol{s}) \in \mathbb{M}_{\tilde{h}, h}\right\},
$$

which becomes $\mathbb{V}_{\tilde{h}, h}=\mathbf{X}_{h}^{m} \times V_{\tilde{h}, h}$, where

$$
V_{\tilde{h}, h}:=\left\{\boldsymbol{\tau} \in \mathbf{X}_{h}^{s}: \quad \int_{\Omega_{s}} \boldsymbol{\tau}: s+\int_{\Sigma} \boldsymbol{\tau} \boldsymbol{n} \cdot \boldsymbol{\varphi}=0 \forall(\boldsymbol{\varphi}, \boldsymbol{s}) \in \mathbb{M}_{\tilde{h}, h}\right\} .
$$

Note that $V_{\tilde{h}, h}$ is not necessarily included in $V$ (cf. (5.36)). Then, using the $3 \mathrm{D}$ version of the equivalence estimate provided by Lemma 5.5 in [12], one can easily show the discrete analogue of (5.43), which means that there exist positive constants $c, h_{0}$, independent of $h$ and $\tilde{h}$, such that for each $\tilde{h} \leq h_{0}$ we have

$$
\begin{aligned}
\operatorname{Re} & \left\{\mathbf{a}_{s}^{+}\left(\mathcal{P}_{s}(\boldsymbol{\sigma}), \mathcal{P}_{s}(\overline{\boldsymbol{\sigma}})\right)+\mathbf{a}_{s}^{+}\left(\left(\mathcal{I}-\mathcal{P}_{s}\right)(\boldsymbol{\sigma}),\left(\mathcal{I}-\mathcal{P}_{s}\right)(\overline{\boldsymbol{\sigma}})\right)\right\} \geq c\|\boldsymbol{\sigma}\|_{\mathbf{H}\left(\operatorname{div} ; \Omega_{s}\right)}^{2} \\
& \forall \boldsymbol{\sigma} \in V_{\tilde{h}, h} .
\end{aligned}
$$

Hence, following verbatim the remaining steps detailed in the proof of Lemma 5.5, we arrive at the discrete version of (5.47); that is, there exists a positive constant $C_{1}$, independent of $h$ and $\tilde{h}$, such that for each $\tilde{h} \leq h_{0}$ we have

$$
\operatorname{Re}\left\{\mathbf{A}_{0}((\boldsymbol{H}, \boldsymbol{\sigma}), \Xi(\overline{\boldsymbol{H}}, \overline{\boldsymbol{\sigma}}))\right\} \geq C_{1}\|(\boldsymbol{H}, \boldsymbol{\sigma})\|_{\mathbb{X}}^{2} \quad \forall(\boldsymbol{H}, \boldsymbol{\sigma}) \in \mathbb{V}_{\tilde{h}, h} .
$$

The discrete inf-sup condition for $\mathbf{A}_{0}$ is then established as follows. 
Lemma 7.8. Let $h_{0}>0$ be the constant mentioned above. Then, there exist constants $C, h_{1}>0$, independent of $h$ and $\tilde{h}$, such that for each $\tilde{h} \leq h_{0}$ and for each $h \leq h_{1}$ we have

$$
\sup _{(\boldsymbol{W}, \boldsymbol{\tau}) \in \mathbb{V}_{\tilde{h}, h} \backslash\{\mathbf{0}\}} \frac{\left|\mathbf{A}_{0}((\boldsymbol{H}, \boldsymbol{\sigma}),(\boldsymbol{W}, \boldsymbol{\tau}))\right|}{\|(\boldsymbol{W}, \boldsymbol{\tau})\|_{\mathbb{X}}} \geq C\|(\boldsymbol{H}, \boldsymbol{\sigma})\|_{\mathbb{X}} \quad \forall(\boldsymbol{H}, \boldsymbol{\sigma}) \in \mathbb{V}_{\tilde{h}, h} .
$$

Proof. Following the definition of the operator $\Xi: \mathbb{X} \rightarrow \mathbb{X}$ (see (5.37) and (5.38)), we now introduce its discrete version as follows:

$$
\begin{aligned}
\Xi_{h}: \mathbb{X}_{h} & \rightarrow \mathbb{X}_{h}, \\
(\boldsymbol{W}, \boldsymbol{\tau}) & \mapsto\left(\left(2 \mathcal{P}_{m, h}-\mathcal{I}\right)(\boldsymbol{W}),\left(2 \mathcal{P}_{s, h}-\mathcal{I}\right)(\boldsymbol{\tau})\right)+\imath \eta(\boldsymbol{W}, \mathbf{0}),
\end{aligned}
$$

where $\eta>0$ is the parameter suitably chosen at the end of the proof of Lemma 5.5. It follows straightforwardly from Lemmas 7.6 and 7.7, redefining $\epsilon:=\min \{\epsilon, 1 / 2\}$, that

$$
\left\|\Xi(\boldsymbol{W}, \boldsymbol{\tau})-\Xi_{h}(\boldsymbol{W}, \boldsymbol{\tau})\right\|_{\mathbb{X}} \leq C_{0} h^{\epsilon}\|(\boldsymbol{W}, \boldsymbol{\tau})\|_{\mathbb{X}} \quad \forall(\boldsymbol{W}, \boldsymbol{\tau}) \in \mathbb{X}_{h}
$$

Hence, using the above estimate, the boundedness of $\mathbf{A}_{0}$, and (7.22), we find that for each $(\boldsymbol{W}, \boldsymbol{\tau}) \in \mathbb{V}_{h, \tilde{h}}$ we have

$$
\begin{aligned}
\operatorname{Re}\{ & \left.\mathbf{A}_{0}\left((\boldsymbol{W}, \boldsymbol{\tau}), \Xi_{h}((\overline{\boldsymbol{W}}, \overline{\boldsymbol{\tau}}))\right)\right\} \\
& \geq \operatorname{Re}\left\{\mathbf{A}_{0}((\boldsymbol{W}, \boldsymbol{\tau}), \Xi((\overline{\boldsymbol{W}}, \overline{\boldsymbol{\tau}})))\right\}-C_{0}\left\|\mathbf{A}_{0}\right\| h^{\epsilon}\|(\boldsymbol{W}, \boldsymbol{\tau})\|_{\mathbb{X}}^{2} \\
& \geq C_{1}\|(\boldsymbol{W}, \boldsymbol{\tau})\|_{\mathbb{X}}^{2}-C_{0}\left\|\mathbf{A}_{0}\right\| h^{\epsilon}\|(\boldsymbol{W}, \boldsymbol{\tau})\|_{\mathbb{X}}^{2} \geq \frac{C_{1}}{2}\|(\boldsymbol{W}, \boldsymbol{\tau})\|_{\mathbb{X}}^{2}
\end{aligned}
$$

for all $\tilde{h} \leq h_{0}$ and for all $h \leq h_{1}:=\left(\frac{C_{1}}{2 C_{0}\left\|\mathbf{A}_{0}\right\|}\right)^{1 / \epsilon}$.

On the other hand, the boundedness of $\mathcal{P}_{s}$ and $\mathcal{P}_{m}$, together with Lemmas 7.6 and 7.7, implies the existence of $C>0$, independent of $h$ and $\tilde{h}$, such that

$$
\left\|\Xi_{h}(\boldsymbol{W}, \boldsymbol{\tau})\right\|_{\mathbb{X}} \leq C\|(\boldsymbol{W}, \boldsymbol{\tau})\|_{\mathbb{X}} \quad \forall(\boldsymbol{W}, \boldsymbol{\tau}) \in \mathbb{X}_{h} .
$$

In addition, it is easy to see, using in particular (7.17), that $\Xi_{h}(\boldsymbol{W}, \boldsymbol{\tau}) \in \mathbb{V}_{h, \tilde{h}}$ for each $(\boldsymbol{W}, \boldsymbol{\tau}) \in \mathbb{V}_{h, \tilde{h}}$. Hence, (7.23) follows immediately from (7.24) and the uniform boundedness of $\Xi_{h}$.

The well-posedness and convergence of the discrete scheme (4.10) can finally be established.

TheOREm 7.1. Assume that the homogeneous problem associated with (4.10) has only the trivial solution. Let $\left.C_{0} \in\right] 0,1\left[\right.$ and $h_{0}, h_{1}>0$ be the constants mentioned above. Then, there exist $\left.\left.\tilde{h}_{0} \in\right] 0, h_{0}\right]$ and $\left.\left.\tilde{h}_{1} \in\right] 0, h_{1}\right]$ such that for each $\tilde{h} \leq \tilde{h}_{0}$ and for each $h \leq \min \left\{\tilde{h}_{1}, C_{0} \tilde{h}\right\}$ the Galerkin scheme (4.10) has a unique solution $\left(\left(\boldsymbol{H}_{h}, \boldsymbol{\sigma}_{h}\right),\left(\boldsymbol{\psi}_{\tilde{h}}, \boldsymbol{r}_{h}\right)\right) \in \mathbb{X}_{h} \times \mathbb{M}_{\tilde{h}, h}$. In addition, there exist $C_{1}, C_{2}>0$, independent of $h$ and $\tilde{h}$, such that

$$
\left\|\left(\left(\boldsymbol{H}_{h}, \boldsymbol{\psi}_{h}\right),\left(\boldsymbol{\psi}_{\tilde{h}}, \boldsymbol{r}_{h}\right)\right)\right\|_{\mathbb{X} \times \mathbb{M}} \leq C_{1}\|(\mathbf{L}, \mathbf{0})\|_{\mathbb{X}^{\prime} \times \mathbb{M}^{\prime}}
$$

and

$$
\begin{aligned}
& \left\|((\boldsymbol{H}, \boldsymbol{\sigma}),(\boldsymbol{\psi}, \boldsymbol{r}))-\left(\left(\boldsymbol{H}_{h}, \boldsymbol{\sigma}_{h}\right),\left(\boldsymbol{\psi}_{\tilde{h}}, \boldsymbol{r}_{h}\right)\right)\right\|_{\mathbb{X} \times \mathbb{M}} \\
& \leq C_{2} \inf _{\left(\left(\boldsymbol{W}_{h}, \boldsymbol{\tau}_{h}\right),\left(\boldsymbol{\varphi}_{\tilde{h}}, \boldsymbol{s}_{h}\right)\right) \in \mathbb{X}_{h} \times \mathbb{M}_{\tilde{h}, h}}\left\|((\boldsymbol{H}, \boldsymbol{\sigma}),(\boldsymbol{\psi}, \boldsymbol{r}))-\left(\left(\boldsymbol{W}_{h}, \boldsymbol{\tau}_{h}\right),\left(\boldsymbol{\varphi}_{\tilde{h}}, \boldsymbol{s}_{h}\right)\right)\right\|_{\mathbb{X} \times \mathbb{M}} .
\end{aligned}
$$

Copyright (c) by SIAM. Unauthorized reproduction of this article is prohibited. 
Furthermore, if there exist $\delta \in(1 / 2,1]$ and $\epsilon \in(0,1]$ such that $\boldsymbol{H} \in \mathbf{H}^{\delta}\left(\mathbf{c u r l}, \Omega_{m}\right)$, $\boldsymbol{\pi}_{\mathbf{t}}(\boldsymbol{H}) \in \mathbf{H}^{\delta}\left(\operatorname{curl}_{\Gamma}, \Gamma\right)$, and $\boldsymbol{u} \in\left[H^{1+\epsilon}\left(\Omega_{s}\right)\right]^{3}$, then there holds

$$
\begin{aligned}
& \left\|((\boldsymbol{H}, \boldsymbol{\sigma}),(\boldsymbol{\psi}, \boldsymbol{r}))-\left(\left(\boldsymbol{H}_{h}, \boldsymbol{\sigma}_{h}\right),\left(\boldsymbol{\psi}_{\tilde{h}}, \boldsymbol{r}_{h}\right)\right)\right\|_{\boldsymbol{H} \times \boldsymbol{Q}} \leq C_{3} \tilde{h}^{\epsilon}\|\boldsymbol{\psi}\|_{\left[H^{1 / 2+\epsilon}(\Sigma)\right]^{3}} \\
& \quad+C_{3} h^{\min (\delta, \epsilon)}\left\{\|\boldsymbol{u}\|_{\left[H^{1+\epsilon}\left(\Omega_{s}\right)\right]^{3}}+\|\boldsymbol{H}\|_{\mathbf{H}^{\delta}\left(\mathbf{c u r l} ; \Omega_{m}\right)}+\left\|\boldsymbol{\pi}_{\mathbf{t}}(\boldsymbol{H})\right\|_{\mathbf{H}^{\delta}\left(\operatorname{curl}_{\Gamma} ; \Gamma\right)}\right\},
\end{aligned}
$$

with a constant $C_{3}>0$, independent of $h$ and $\tilde{h}$.

Proof. Thanks to Lemmas 7.5 and 7.8, the first part of the proof is a direct application of Theorem 13.7 in [18], whereas the rate of convergence (7.27) follows from the Cea estimate (7.26) and the approximation properties of the finite element subspaces provided in section 7.1 (cf. (7.6), $\left(\mathrm{AP}_{h}^{\sigma}\right),\left(\mathrm{AP}_{\tilde{h}}^{\psi}\right)$, and $\left.\left(\mathrm{AP}_{h}^{r}\right)\right)$.

8. Numerical results. In this section we present an example illustrating the performance of the finite element scheme (6.4) on a set of uniform meshes of the domain. We begin by introducing some notation. The variable $N$ stands for the global number of degrees of freedom involved in our Galerkin method, and the individual errors are denoted by

$$
\begin{gathered}
e(\boldsymbol{H}):=\left\|\boldsymbol{H}-\boldsymbol{H}_{h}\right\|_{\mathbf{H}\left(\mathbf{c u r l} ; \Omega_{m}\right)}, \quad e(\boldsymbol{\sigma}):=\left\|\boldsymbol{\sigma}-\boldsymbol{\sigma}_{h}\right\|_{\mathbf{H}\left(\mathbf{d i v} ; \Omega_{s}\right)}, \\
e(\boldsymbol{u}):=\left\|\boldsymbol{u}-\boldsymbol{u}_{h}\right\|_{\left[L^{2}\left(\Omega_{s}\right)\right]^{3}}, \quad e(\boldsymbol{r}):=\left\|\boldsymbol{r}-\boldsymbol{r}_{h}\right\|_{\left[L^{2}\left(\Omega_{s}\right)\right]^{3 \times 3},}
\end{gathered}
$$

where, as suggested by (4.5), $\boldsymbol{u}_{h}$ is computed as

$$
\boldsymbol{u}_{h}:=-\frac{1}{\kappa_{s}^{2}} \operatorname{div}\left(\boldsymbol{\sigma}_{h}\right) \quad \text { in } \Omega_{s} .
$$

Also, we let $r(\boldsymbol{H}), r(\boldsymbol{\sigma}), r(\boldsymbol{u})$, and $r(\boldsymbol{r})$ be the corresponding experimental rates of convergence. In particular,

$$
r(\boldsymbol{H}):=\frac{\log \left(e(\boldsymbol{H}) / e^{\prime}(\boldsymbol{H})\right)}{\log \left(h / h^{\prime}\right)},
$$

where $h$ and $h^{\prime}$ denote two consecutive mesh sizes with corresponding errors $e$ and $e^{\prime}$, and similar definitions hold for the rest of the variables.

We now describe the data of the example. We consider the domains $\Omega_{s}:=$ $(0.25,0.75)^{3}$ and $\Omega_{m}:=(0,1)^{3} \backslash[0.25,0.75]^{3}$, and take the solid parameters $\rho_{s}=$ $\lambda_{s}=\mu_{s}=1$ and the electromagnetic parameters $\epsilon=\epsilon_{0}=\mu=\mu_{0}=1$. We take the frequency $\omega=3$ and $\sigma=0$, whence $\kappa_{s}=k=3$ and $a=b=1$. The solution of the elastodynamic equation is given by

$$
\boldsymbol{u}(\mathbf{x})=\left(\begin{array}{c}
\sin \left(\pi x_{1}\right) \sin \left(\pi x_{2}\right) \sin \left(\pi x_{3}\right) \\
\exp \left(x_{1}\right) \exp \left(x_{2}\right) x_{3} \\
\exp \left(x_{2}\right)
\end{array}\right)(1+\iota) \quad \forall \mathbf{x}:=\left(x_{1}, x_{2}, x_{3}\right)^{\mathrm{t}} \in \Omega_{s}
$$

whereas the function

$$
\boldsymbol{H}(\mathbf{x}):=\operatorname{curl}\left(\frac{\exp \left(\iota k R_{m}\right)}{R_{m}}, 0,0\right) \quad \forall \mathbf{x}:=\left(x_{1}, x_{2}, x_{3}\right)^{\mathrm{t}} \in \Omega_{m},
$$

with $R_{m}:=\sqrt{\left(x_{1}-0.5\right)^{2}+\left(x_{2}-0.5\right)^{2}+\left(x_{3}-0.5\right)^{2}}$, solves the first equation of (3.12) in $\Omega_{m}$. It follows that $(\boldsymbol{u}, \boldsymbol{H})$ is solution of (3.12) with nonhomogeneous righthand side of the elastodynamic equation, nonhomogeneous transmission conditions on $\Sigma$, and suitable essential boundary conditions on $\Gamma$. 
According to the requirements established in our main result, Theorem 7.1, for the mesh sizes $h$ and $\tilde{h}$, and since the constant $C_{0}$ mentioned there is not explicitly known, we simply put a vertex of the independent partition $\mathcal{T}_{\tilde{h}}(\Sigma)$ every two vertices of $\mathcal{T}_{h}$ on $\Sigma$. As we will see below, this choice works out well in the present example since no spurious solutions appear. In addition, there is no need to take sufficiently small values of $\tilde{h}$ and $h$ (as technically suggested by the inequalities $\tilde{h} \leq \tilde{h}_{0}$ and $h \leq \tilde{h}_{1}$ in Theorem 7.1) since the resulting discrete schemes become all well-posed for the degrees of freedom employed.

In Table 1 we summarize the convergence history for a sequence of uniform meshes of the domain $\bar{\Omega}_{s} \cup \bar{\Omega}_{m}$. We observe that $e(\boldsymbol{H})$ constitutes the dominant part of the total error, and that the order of convergence provided by Theorem 7.1 when $\delta=\epsilon=1$, that is, $O(h)$, is fully attained by all the unknowns. Moreover, we find that the convergence of $\boldsymbol{u}$ and $\boldsymbol{r}$ is a bit faster than $O(h)$, which could mean either a superconvergence phenomenon or a special feature of this particular example.

TABLE 1

Mesh sizes, degrees of freedom, individual errors, and rates of convergence $\left(\omega=k=\kappa_{s}=3\right)$.

\begin{tabular}{r|c||c|c||c|c}
\hline$h$ & $N$ & $e(\boldsymbol{H})$ & $r(\boldsymbol{H})$ & $e(\boldsymbol{\sigma})$ & $r(\boldsymbol{\sigma})$ \\
\hline $1 / 4$ & 1043 & $1.166 \mathrm{E}+01$ & - & $2.743 \mathrm{E}-00$ & - \\
$1 / 8$ & 6913 & $6.794 \mathrm{E}+00$ & 0.779 & $1.565 \mathrm{E}-00$ & 0.810 \\
$1 / 12$ & 21843 & $4.743 \mathrm{E}+00$ & 0.887 & $9.913 \mathrm{E}-01$ & 1.126 \\
$1 / 16$ & 50057 & $3.627 \mathrm{E}+00$ & 0.932 & $7.307 \mathrm{E}-01$ & 1.061 \\
$1 / 20$ & 95779 & $2.931 \mathrm{E}+00$ & 0.955 & $5.773 \mathrm{E}-01$ & 1.056 \\
$1 / 24$ & 163233 & $2.457 \mathrm{E}+00$ & 0.968 & $4.772 \mathrm{E}-01$ & 1.045 \\
$1 / 28$ & 256643 & $2.113 \mathrm{E}+00$ & 0.976 & $4.068 \mathrm{E}-01$ & 1.036 \\
\hline \hline$h$ & $N$ & $e(\boldsymbol{u})$ & $r(\boldsymbol{u})$ & $e(\boldsymbol{r})$ & $r(\boldsymbol{r})$ \\
\hline $1 / 4$ & 1043 & $8.625 \mathrm{E}-01$ & - & $3.350 \mathrm{E}-01$ & - \\
$1 / 8$ & 6913 & $7.717 \mathrm{E}-01$ & 0.161 & $2.486 \mathrm{E}-01$ & 0.430 \\
$1 / 12$ & 21843 & $3.934 \mathrm{E}-01$ & 1.662 & $1.270 \mathrm{E}-01$ & 1.656 \\
$1 / 16$ & 50057 & $2.597 \mathrm{E}-01$ & 1.443 & $8.446 \mathrm{E}-02$ & 1.418 \\
$1 / 20$ & 95779 & $1.852 \mathrm{E}-01$ & 1.516 & $5.919 \mathrm{E}-02$ & 1.594 \\
$1 / 24$ & 163233 & $1.405 \mathrm{E}-01$ & 1.515 & $4.369 \mathrm{E}-02$ & 1.665 \\
$1 / 28$ & 256643 & $1.113 \mathrm{E}-01$ & 1.509 & $3.367 \mathrm{E}-02$ & 1.690 \\
\hline
\end{tabular}

Acknowledgment. The authors are thankful to Antonio Márquez for performing the computational code and running the numerical example.

\section{REFERENCES}

[1] A. Alonso And A. VAlli, An optimal domain decomposition preconditioner for low-frequency time-harmonic Maxwell equations, Math. Comput., 68 (1999), pp. 607-631.

[2] C. Amrouche, C. Bernardi, M. Dauge, and V. Girault, Vector potentials in threedimensional nonsmooth domains, Math. Methods Appl. Sci., 21 (1998), pp. 823-864.

[3] D. N. Arnold, F. Brezzi, and J. Douglas, PEERS: A new mixed finite element method for plane elasticity, Japan J. Appl. Math., 1 (1984), pp. 347-367.

[4] A. Buffa, Remarks on the discretization of some noncoercive operator with applications to heterogeneous Maxwell equations, SIAM J. Numer. Anal., 43 (2005), pp. 1-18.

[5] A. Buffa And P. Ciarlet, JR., On traces for functional spaces related to Maxwell's equation. Part I: An integration by parts formula in Lipschitz polyhedra, Math. Methods Appl. Sci., 24 (2001), pp. 9-30.

[6] A. Buffa AND P. Ciarlet, JR., On traces for functional spaces related to Maxwell's equation. Part II: Hodge decompositions on the boundary of Lipschitz polyhedra and applications, Math. Methods Appl. Sci., 21 (2001), pp. 31-48.

[7] A. Buffa, M. Costabel, and D. Sheen, On traces for $\mathrm{H}(\mathrm{curl}, \Omega)$ in Lipschitz domains, J. Math. Anal. Appl., 276 (2002), pp. 845-867. 
[8] A. Buffa And R. Hiptmair, Galerkin boundary element methods for electromagnetic scattering, Topics in Computational Wave Propagation, Lectures Notes Comput. Sci. Engrg. 31, Springer, Berlin, 2003, pp. 83-124.

[9] F. Brezzi And M. Fortin, Mixed and Hybrid Finite Element Methods, Springer-Verlag, New York, 1991.

[10] F. CAKONI ANd G. C. Hsiao, Mathematical model of the interaction problem between electromagnetic field and elastic body, in Acoustics, Mechanics, and the Related Topics of Mathematical Analysis, World Scientific Publishing, River Edge, NJ, 2002, pp. 48-54.

[11] M. Dauge, Elliptic Boundary Value Problems on Corner Domains: Smoothness and Asymptotics of Solutions, Lecture Notes in Math. 1341, Springer, Berlin, 1988.

[12] G. N. Gatica, A. Márquez, and S. Meddahi, Analysis of the coupling of primal and dualmixed finite element methods for a two-dimensional fluid-solid interaction problem, SIAM J. Numer. Anal., 45 (2007), pp. 2072-2097.

[13] G. N. Gatica, A. Márquez, and S. Meddahi, A new dual-mixed finite element method for the plane linear elasticity problem with pure traction boundary conditions, Comput. Methods Appl. Mech. Engrg., 197 (2008), pp. 1115-1130.

[14] G. N. Gatica, A. Márquez, and S. Meddahi, Analysis of the coupling of BEM, FEM, and mixed-FEM for a two-dimensional fluid-solid interaction problem, Appl. Numer. Math., 59 (2009), pp. 2735-2750.

[15] V. Girault And P.-A. Raviart, Finite Element Methods for Navier-Stokes Equations. Theory and Algorithms, Springer, Berlin, 1986.

[16] R. Hiptmair, Finite elements in computational electromagnetism, Acta Numer., 11 (2002), pp. 237-339.

[17] R. Hiptmair, Coupling of finite elements and boundary elements in electromagnetic scattering, SIAM J. Numer. Anal., 41 (2003), pp. 919-944.

[18] R. KRess, Linear Integral Equations, Springer-Verlag, Berlin, 1989.

[19] M. LONSING AND R. VerfürTh, On the stability of BDMS and PEERS elements, Numer. Math., 99 (2004), pp. 131-140.

[20] G. A. Maugin, Continuum Mechanics of Electromagnetic Solids, North-Holland, Amsterdam, 1988.

[21] P. Monk, Finite Element Methods for Maxwell's Equations, Oxford University Press, New York, 2003.

[22] A. Quarteroni and A. Valli, Domain Decomposition Methods for Partial Differential Equations. Numerical Mathematics and Scientific Computation, Oxford Science Publications, The Clarendon Press, Oxford University Press, New York, 1999.

[23] J. Xu And L. Zikatanov, Some observations on Babuška and Brezzi theories, Numer. Math., 94 (2003), pp. 195-202.

Copyright (C) by SIAM. Unauthorized reproduction of this article is prohibited. 